Supporting Information

\title{
Photocatalytic Umpolung Synthesis of Nucleophilic $\pi$-allylcobalt Complexes for Allylation of Aldehydes
}

Caizhe Shi ${ }^{\mathrm{a}}$, Fusheng $\mathrm{Li}^{\mathrm{a}}$, Yuqing Chen ${ }^{\mathrm{a}}$, Shuangjie Lin ${ }^{\mathrm{a}}$, Erjun Hao ${ }^{\mathrm{b}}$, Zhuowen Guo $^{\mathrm{a}}$, Urwa Tul Wosqa ${ }^{\mathrm{a}}$, Dandan Zhang ${ }^{\mathrm{a}}$, and Lei Shi ${ }^{* a, b}$

${ }^{a}$ Dalian University of Technology, Zhang Dayu School of Chemistry, State Key Laboratory of Fine Chemicals, Dalian, Liaoning, 116024, China.

\footnotetext{
${ }^{b}$ Henan Key Laboratory of Organic Functional Molecules and Drug Innovation, Key Laboratory of Green Chemical Media and Reactions, Ministry of Education, School of Chemistry and Chemical Engineering, Henan Normal University, Xinxiang, Henan 453007, China.
}

Email: shilei17@dlut.edu.cn 


\section{Table of Contents}

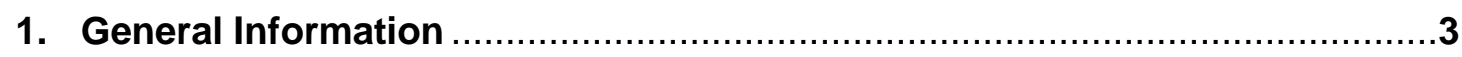

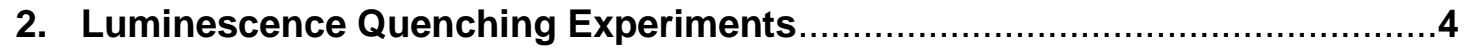

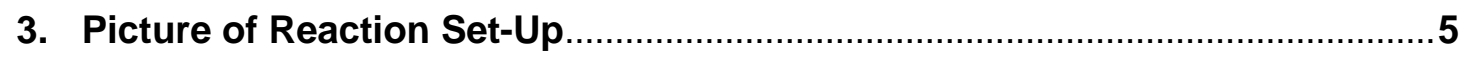

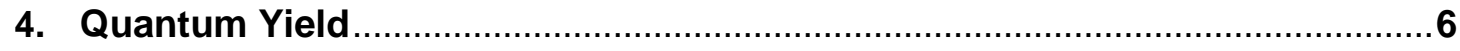

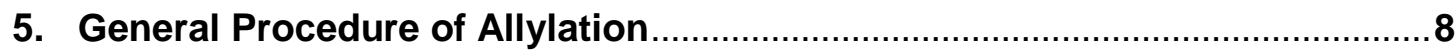

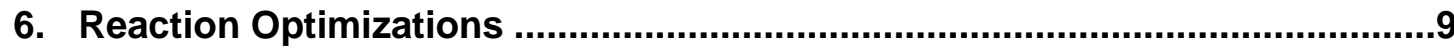

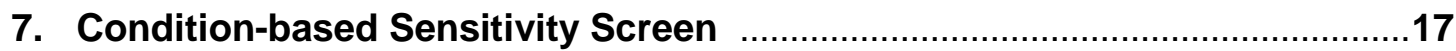

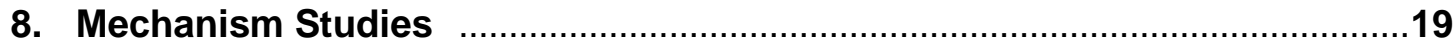

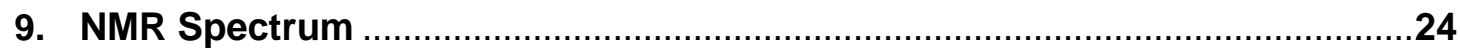




\section{S1 General Information}

Unless otherwise noted, all reactions of substrates preparation were conducted in flame dried glassware under a nitrogen atmosphere using anhydrous solvent passed through an activated alumina column (Innovative Technology). Commercially available reagents were used without further purification. Thin layer chromatography (TLC) was performed using Huanghai TLC silica gel plates HSG F254 and visualized using UV light, anisaldehyde or potassium permanganate. The photocatalytic reactions were performed on WATTCAS Parallel Light Reactor (WP - TEC - 1020L) with 10W LED. ${ }^{1} \mathrm{H}$ and ${ }^{13} \mathrm{C}$ NMR spectra were recorded in $\mathrm{CDCl}_{3}$ on a Bruker 400M spectrometer. Chemical shifts in ${ }^{1} \mathrm{H}$ NMR spectra were reported in parts per million (ppm) on the $\delta$ scale from an internal standard of residual $\mathrm{CDCl}_{3}(7.26 \mathrm{ppm})$. Data for ${ }^{1} \mathrm{H}$ NMR were reported as follows: chemical shift, multiplicity $(\mathrm{s}=$ singlet, $\mathrm{d}=$ doublet, $\mathrm{t}$ = triplet, $\mathrm{q}=$ quartet, $\mathrm{m}=$ multiplet, $\mathrm{br}=$ broad), coupling constant in $\mathrm{Hertz}(\mathrm{Hz})$ and integration . Data for ${ }^{13} \mathrm{C}$ NMR spectra were reported in terms of chemical shift in ppm from the central peak of $\mathrm{CDCl}_{3}(77.00 \mathrm{ppm})$. ESI mass spectra were obtained from an HPLC - Q - Tof mass spectrometer using acetonitrile as the mobile phase. UV - vis spectra were collected on an HP 8453 spectrometer. The fluorescence emission spectra were collected on an Edinburgh FS920. 


\section{S2 Luminescence Quenching Experiments}

Emission intensities were recorded using Edinburgh FS920 Fluorescense Spectrophotometer for all experiments. All $\operatorname{Ir}(\mathrm{ppy})_{2}(\mathrm{dtbbpy}) \mathrm{PF}_{6}$ soltions were excited at $450 \mathrm{~nm}$ and the emission intensity was collected at $500-800 \mathrm{~nm}$. In a typical experiment, the DMF solution of $\operatorname{Ir}(\mathrm{ppy})_{2}(\mathrm{dtbbpy}) \mathrm{PF}_{6}\left(10^{-5} \mathrm{M}\right)$ was added the appropriate amount of quencher in a screw - top $4.5 \mathrm{~cm}$ quartz cuvette. After degassing with nitrogen for $15 \mathrm{~min}$, the emission spectra of the sample were collected.

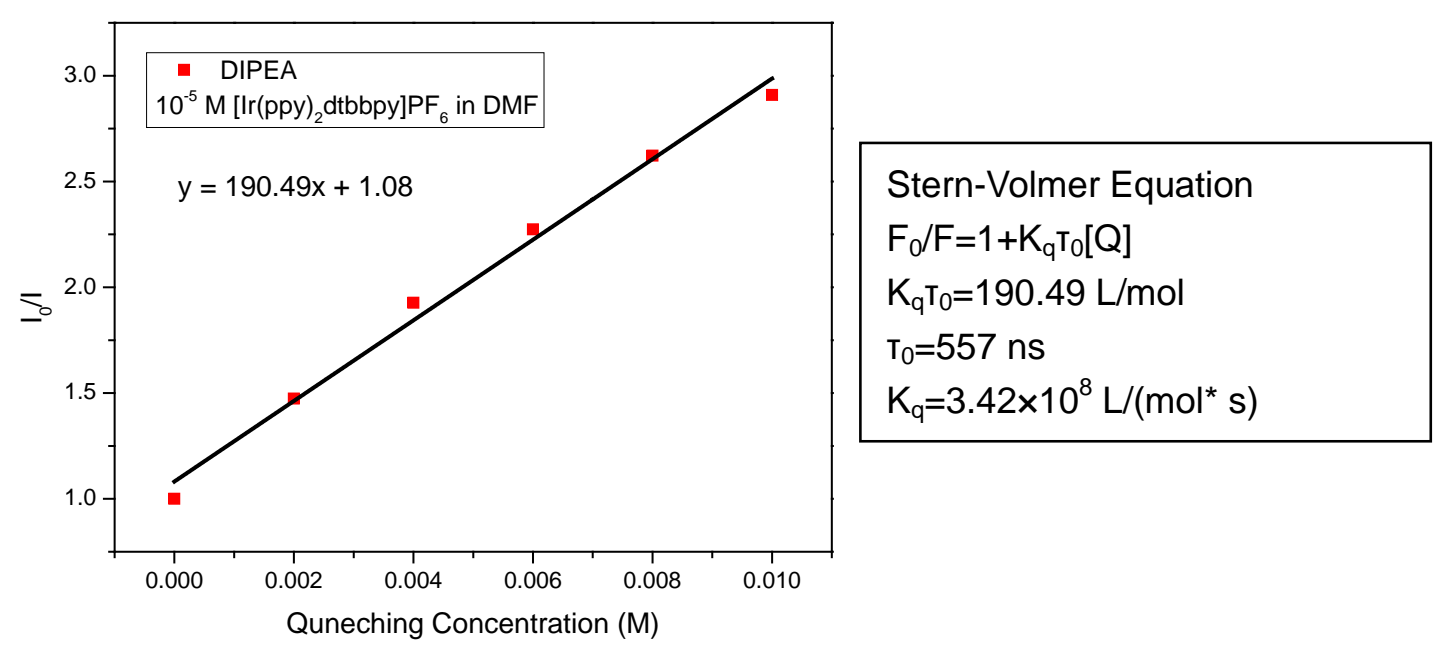

Figure S1. $\operatorname{Ir}(\mathrm{ppy})_{2}(\mathrm{dtbbpy}) \mathrm{PF}_{6}$ emission quenching by DIPEA.

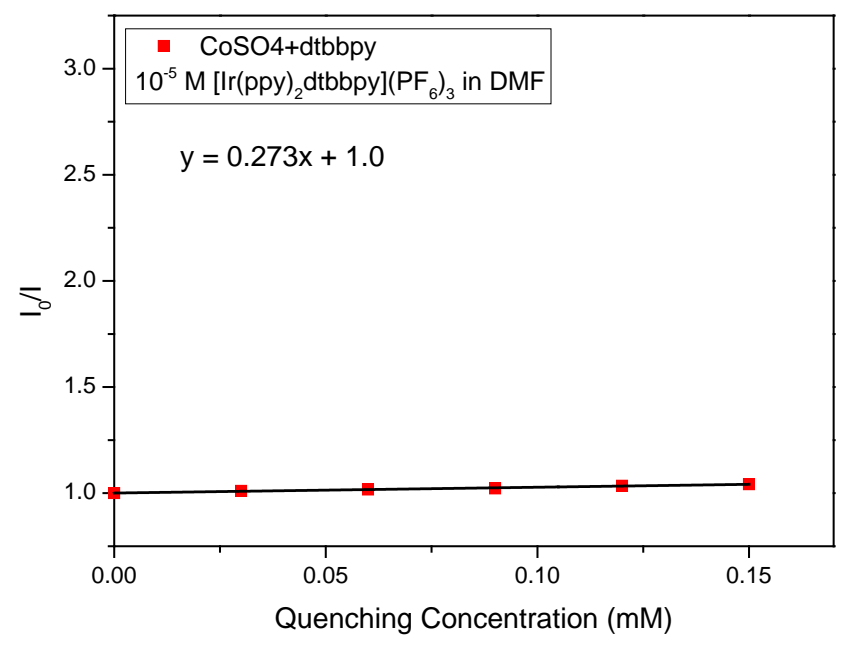

Figure S2. $\operatorname{Ir}(\mathrm{ppy})_{2}(\mathrm{dtbbpy}) \mathrm{PF}_{6}$ emission quenching by $\mathrm{CoSO}_{4}$ and dtbbpy. 
S3 Picture of Reaction Set - Up

The photocatalytic reactions were performed on WATTCAS Parallel Light Reactor (WP - TEC $-1020 L)$.

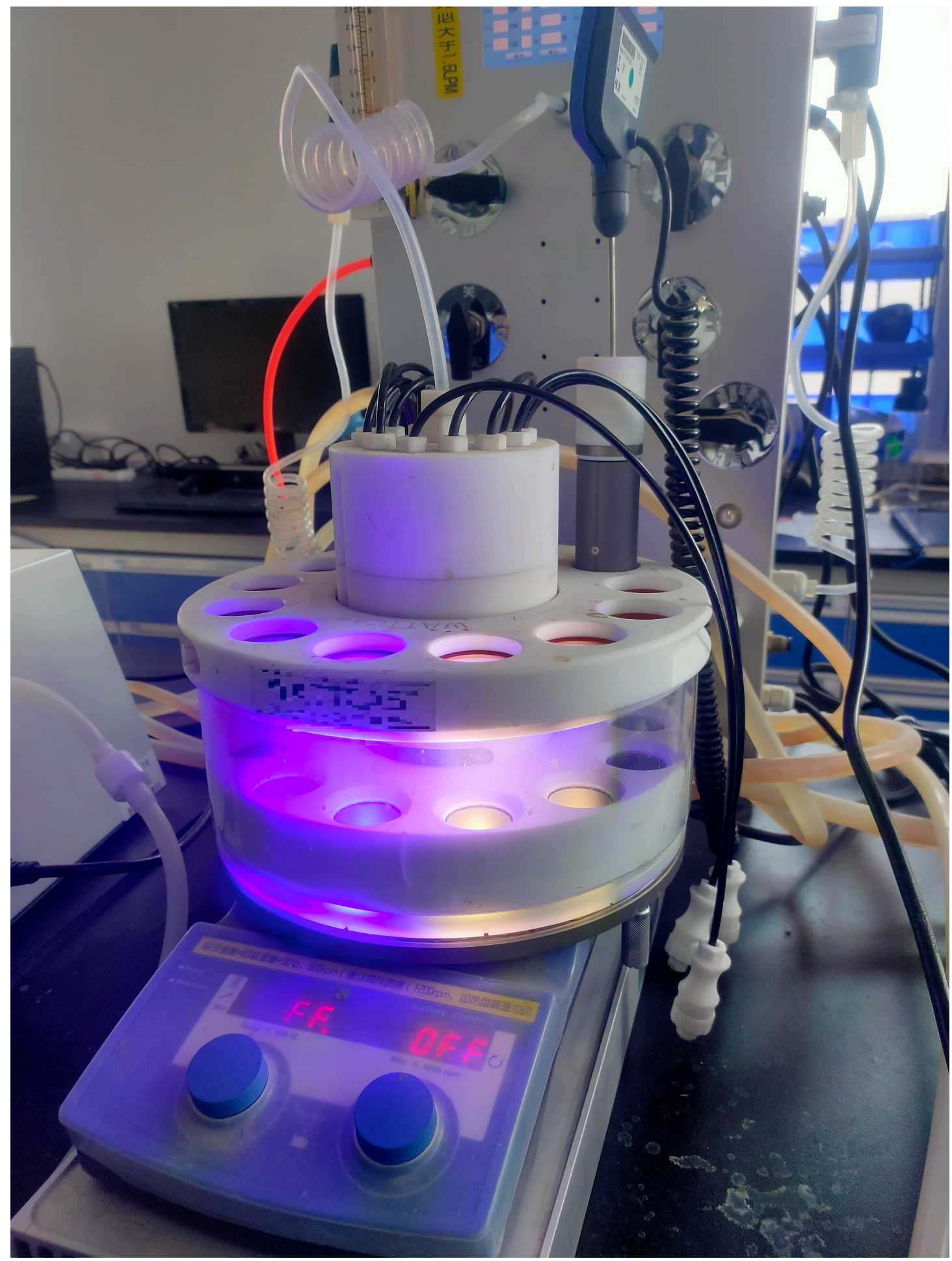




\section{S4 Quantum Yield}

The photocatalytic reactions were performed on WATTCAS Parallel Light Reactor (WP - TEC - 1020SL) with blue COB LED $450-455 \mathrm{~nm}$. After irradiation, the yield of alcohol $2 \mathrm{e}$ was determined by ${ }^{1} \mathrm{H}$ NMR based on a 1,3,5 - trimethoxybenzene standard and the yield was $11 \%$ after 3.5 hrs.

The energy of a photon $E_{p h}$ at a wavelength $\lambda$ is calculated as

$E_{p h}=\mathrm{hc} / \lambda=4.388 \times 10^{-19} \mathrm{~J}$

$\lambda$ : wavelength $\left(\lambda=4.53 \times 10^{-7} \mathrm{~m}\right)$

$\mathrm{h}$ : planck constant $\left(\mathrm{h}=6.626 \times 10^{-34} \mathrm{~J}^{*} \mathrm{~s}\right)$

c:velocity of light $\left(\mathrm{c}=3 \times 10^{8} \mathrm{~m} / \mathrm{s}\right)$

The total photon flux $F_{p h}$ is calculated as

$F_{p h}=\mathrm{fP} / E_{p h}=4.44 \times 10^{18} \mathrm{~S}^{-1}$

$\mathrm{f}: 1-10^{-\mathrm{A}}$ considering that in the reaction condition the number of transmitted photons is negligible in the whole range of emission of the lamp, it can be assumed that all photons are absorbed by the photocatalyst.

$P: E^{\star} S$ (E: illumination intensity, $E=0.975 \mathrm{~W} / \mathrm{cm}^{2}$; $S$ : the area irradiated, $S=\pi r^{2}=2.0 \mathrm{~cm}^{2}$

Quantum yield was determined as:

$\boldsymbol{\phi}=$ Mole number for the product/Mole number photons absorbed

$=0.11 \times 10^{-3} \times 6.02 \times 10^{23} / F_{p h} \times 1260 s=0.012$

This result revealed that the main pathway of this reaction was not a photo - initiated radical chain process, but a photocatalyzed process. 
CLED Test Report

\begin{tabular}{|l|l|}
\hline Name & Value \\
\hline Dominant(nm) & 458.5 \\
\hline Purity(\%) & 99 \\
\hline HalfWidth(nm) & 22.2 \\
\hline Peak(nm) & 454.6 \\
\hline Center(nm) & 454.6 \\
\hline Color Ratio(RGB) & $0.0,7.5,92.5$ \\
\hline Ee(nW/cm $\left.{ }^{2}\right)$ & 975.913 \\
\hline
\end{tabular}


S5 General Procedure of Allylation Reacrion<smiles>[R]C=O</smiles>

1<smiles>C=CCOC(C)=O</smiles><smiles>C[14CH2]</smiles><smiles>[R]C(O)CC=C</smiles>

2

Following the standard procedure, a solution of 1 ( $0.5 \mathrm{mmol}, 1 \mathrm{eq}$ ), allyl acetate $(1.5 \mathrm{mmol}, 3$ eq.), DIPEA (1.5 mmol, 3 eq.), $\mathrm{CoSO}_{4} \bullet \mathrm{H}_{2} \mathrm{O}$ (10 mol\%), dtbbpy $(10 \mathrm{~mol} \%)$ and $\operatorname{Ir}(\mathrm{ppy})_{2}(\mathrm{dtbbpy}) \mathrm{PF}_{6}(1 \mathrm{~mol} \%)$ in DMF $(0.2 \mathrm{M})$. The reaction mixture was stirred at room temperature with irradiation of $10 \mathrm{~W} 450 \mathrm{~nm}$ LED for $32 \mathrm{~h}$. The ${ }^{1} \mathrm{H}$ NMR analysis of the crude product was calculated using 1,3,5 - Trimethyoxybenzene as the internal standard. Yields are shown in manuscripts table 2 and table 3. 
S6 Reaction Optimizations

Table S1 Screen of solvents

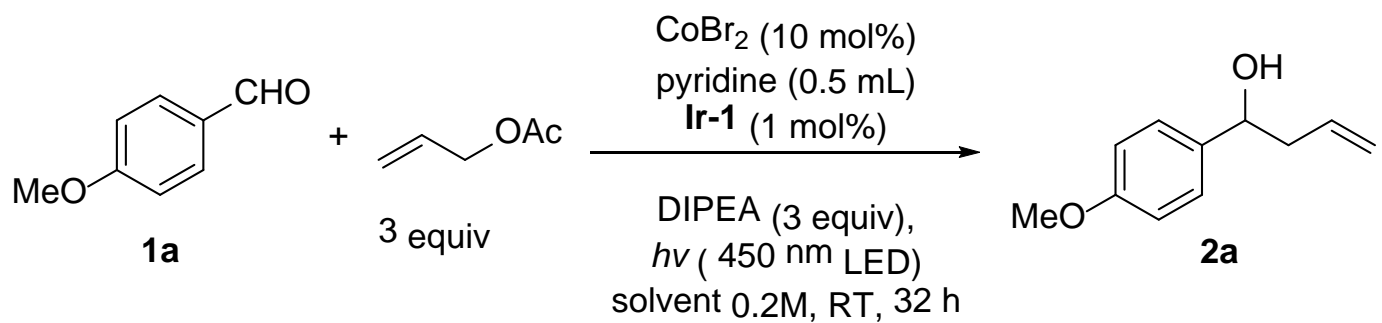

\begin{tabular}{|c|c|c|}
\hline \multirow{2}{*}{ Entry } & Solvents & Yield(\%) \\
\cline { 3 - 3 } & & $\mathbf{2 a}$ \\
\hline 1 & $\mathrm{CH}_{3} \mathrm{CN}$ & 16 \\
\hline 2 & $\mathrm{CH}_{2} \mathrm{Cl}_{2}$ & trace \\
\hline 3 & $\mathrm{THF}$ & 19 \\
\hline 4 & $\mathrm{DCE}$ & 12 \\
\hline 5 & $\mathrm{DMF}$ & 29 \\
\hline
\end{tabular}


Table S2 Solution Concentration

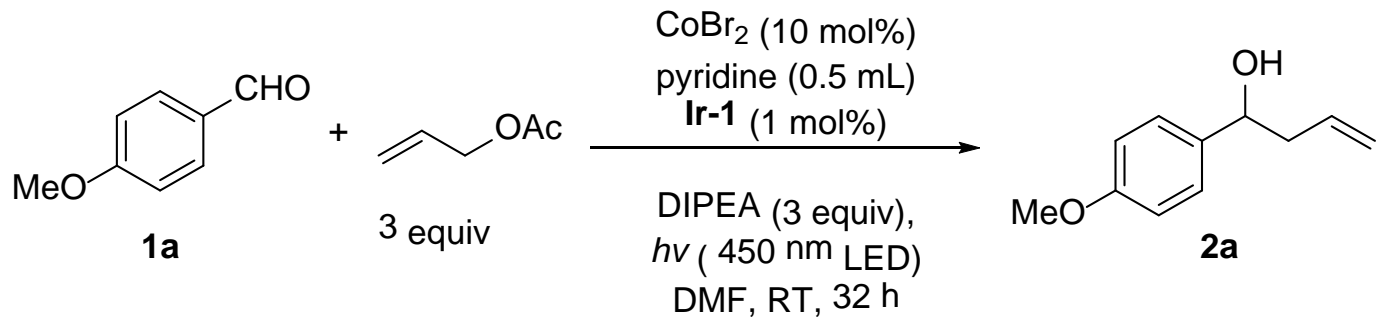

\begin{tabular}{|c|c|c|}
\hline \multirow{2}{*}{ Entry } & Concentration & Yield(\%) \\
\cline { 3 - 3 } & & $2 \mathrm{a}$ \\
\hline 1 & $0.1 \mathrm{M}$ & 22 \\
\hline 2 & $0.3 \mathrm{M}$ & 19 \\
\hline 3 & $0.5 \mathrm{M}$ & 18 \\
\hline
\end{tabular}


Table S3 Screen of ligands

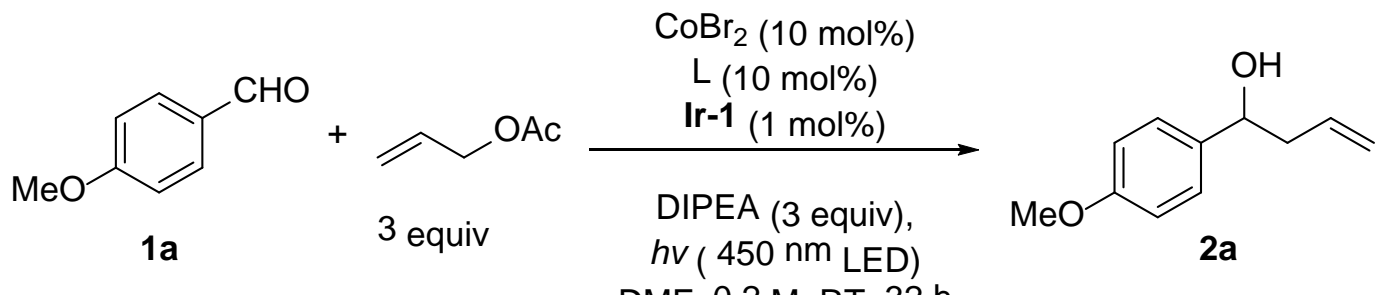

DMF, $0.2 \mathrm{M}, \mathrm{RT}, 32 \mathrm{~h}$

\begin{tabular}{|c|c|c|}
\hline \multirow{2}{*}{ Entry } & \multirow{2}{*}{ Ligands } & Yield(\%) \\
\hline & & $2 a$ \\
\hline 1 & No ligand & trace \\
\hline 2 & L1 & 34 \\
\hline 3 & L2 & 37 \\
\hline 4 & L3 & 58 \\
\hline 5 & L4 & 15 \\
\hline 6 & L8 & 25 \\
\hline 7 & L6 & 14 \\
\hline 8 & L7 & trace \\
\hline 9 & L8 & trace \\
\hline 10 & L9 & trace \\
\hline
\end{tabular}




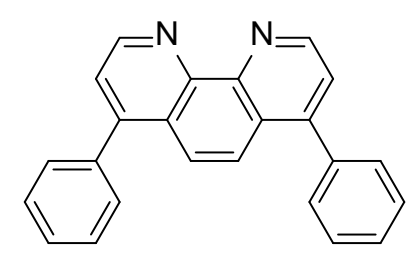

L1

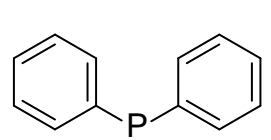<smiles>Cc1ccccc1</smiles>

L4

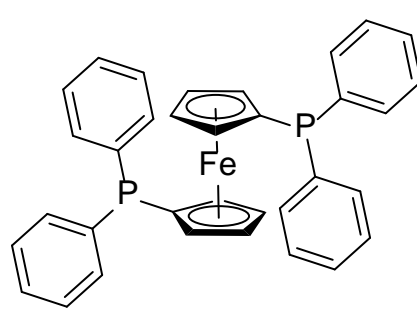

L7

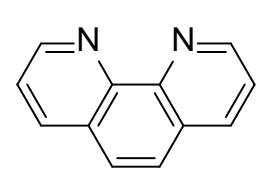

L2<smiles>c1ccc(P(CCP(c2ccccc2)c2ccccc2)c2ccccc2)cc1</smiles>

L5

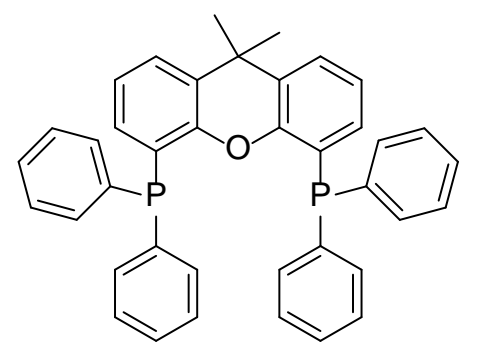

L8
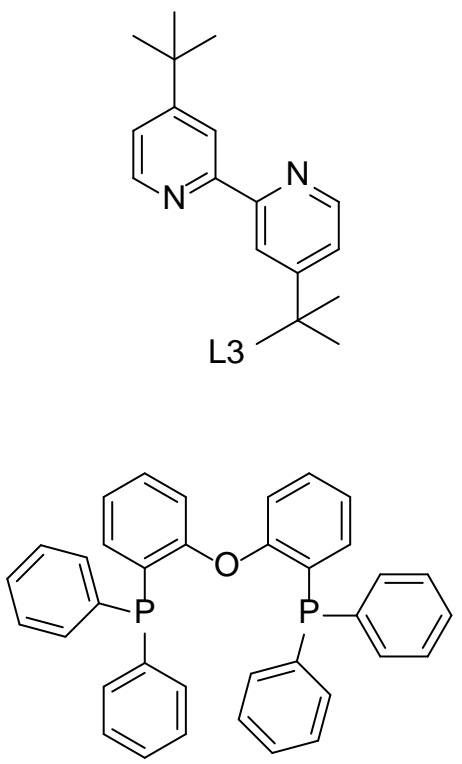

L6

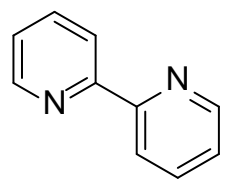

L9 
Table S4 Screen of photocatalysts

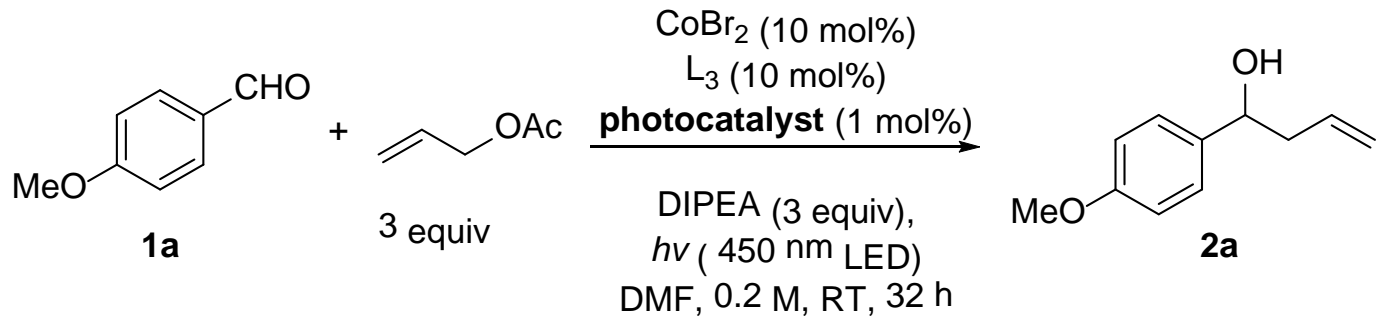

\begin{tabular}{|c|c|c|}
\hline \multirow{2}{*}{ Entry } & Photocatalysts & Yield(\%) \\
\cline { 2 - 3 } & & $\mathbf{2 a}$ \\
\hline 1 & $4 \mathrm{C}_{Z}$ IPN & trace \\
\hline 2 & $\mathbf{R u}-\mathbf{1}$ & $<5$ \\
\hline 3 & $\mathbf{I r}-\mathbf{2}$ & 14 \\
\hline
\end{tabular}

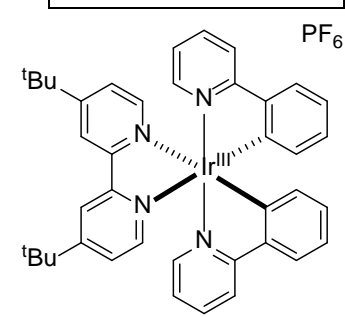

$\operatorname{Ir}($ ppy) 2 (dtbbpy)PF 6 Ir-1

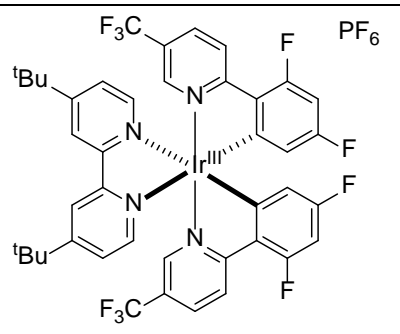

$\operatorname{Ir}\left[\mathrm{dF}\left(\mathrm{CF}_{3}\right)\right.$ ppy2](dtbbpy)PF 6 Ir-2

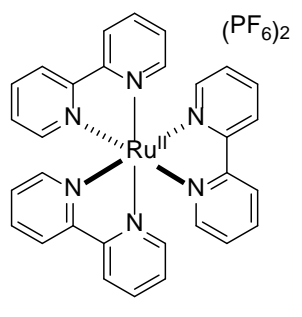

$\mathrm{Ru}(\mathrm{bpy})_{3}\left(\mathrm{PF}_{6}\right)_{2}$ Ru-1

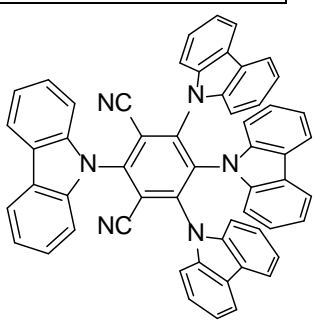

4CZIPN 
Table S5 Screen of various cobalt salts

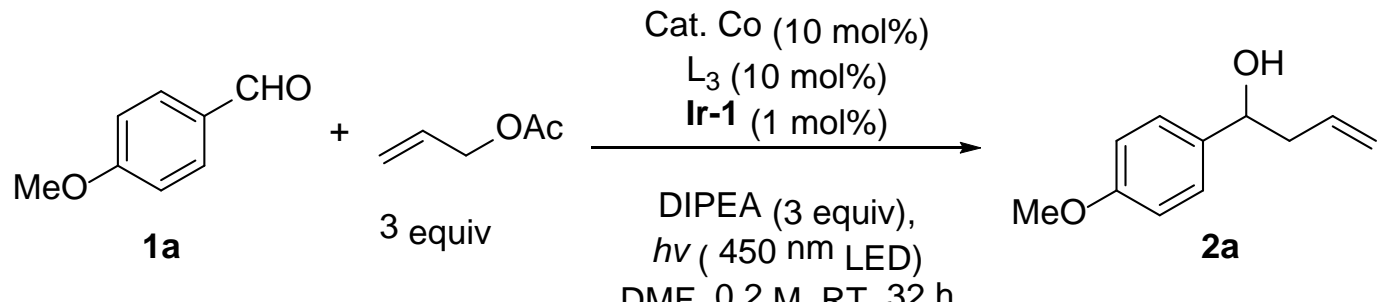

DMF, 0.2 M, RT, $32 \mathrm{~h}$

\begin{tabular}{|c|c|c|}
\hline \multirow{2}{*}{ Entry } & Cobalt Salts & Yield(\%) \\
\cline { 3 - 3 } & & $\mathbf{2 a}$ \\
\hline 1 & No cobalt salt & 0 \\
\hline 2 & $\mathrm{CoCl}_{2}$ & 55 \\
\hline 3 & $\mathrm{Co}\left(\mathrm{BF}_{4}\right)_{2} \bullet 6 \mathrm{H}_{2} \mathrm{O}$ & 61 \\
\hline 4 & $\mathrm{CoSO}_{4} \bullet \mathrm{H}_{2} \mathrm{O}$ & 80 \\
\hline 5 & $\mathrm{Co}(\mathrm{OAc})_{2} \bullet 4 \mathrm{H}_{2} \mathrm{O}$ & 75 \\
\hline 6 & $\mathrm{Co}\left(\mathrm{NO}_{3}\right)_{2} \bullet 6 \mathrm{H}_{2} \mathrm{O}$ & $<5$ \\
\hline 7 & $\mathrm{Co}(\mathrm{acac})_{3}$ & 17 \\
\hline
\end{tabular}


Table S6 Screen of organic electron donors

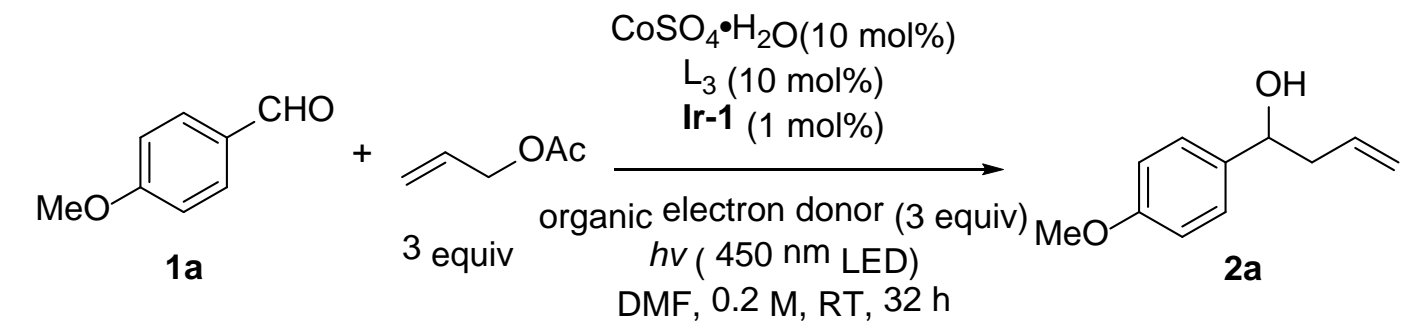

\begin{tabular}{|c|c|c|}
\hline \multirow{2}{*}{ Entry } & Organic Electron Donors & Yield(\%) \\
\cline { 3 - 3 } & & $\mathbf{2 a}$ \\
\hline 1 & No organic electron donors & 0 \\
\hline 2 & $\mathrm{HE}$ & 0 \\
\hline
\end{tabular}


Table S7 Screen of additives

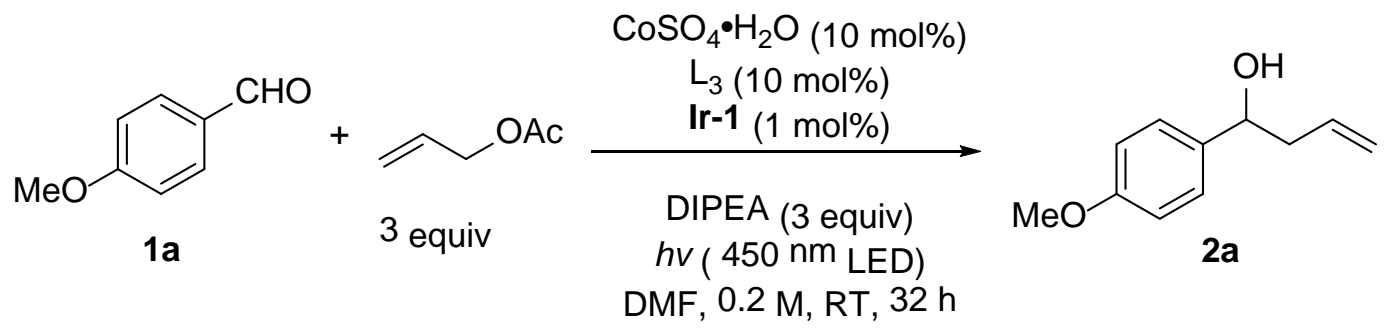

\begin{tabular}{|c|c|c|}
\hline \multirow{2}{*}{ Entry } & \multirow{2}{*}{ Additives } & Yield(\%) \\
\cline { 2 - 3 } & & $\mathbf{2 a}$ \\
\hline 1 & $\mathrm{CF}_{3} \mathrm{COOH}$ & 42 \\
\hline 2 & $\mathrm{~K}_{2} \mathrm{CO}_{3}$ & 40 \\
\hline 3 & $\mathrm{ZnCl}_{2}$ & 30 \\
\hline
\end{tabular}




\section{S7 Condition-based Sensitivity Screen}

Results of the Sensitivity Assessment of Reaction p-anisaldehyde with tert-butyl cinnamyl carbonate:<smiles>COc1ccc(C=O)cc1</smiles>

3 eq.

$$
\mathrm{CoSO}_{4} \cdot \mathrm{H}_{2} \mathrm{O}(10 \mathrm{~mol} \%)
$$

$$
\mathrm{L}_{3}(10 \mathrm{~mol} \%)
$$

Ir-1 (1 mol\%)

DIPEA (3 eq.)

hv (450 nm LED)

DMF, RT, $32 \mathrm{~h}$<smiles>C=CC(c1ccccc1)[C@H](O)c1ccc(OC)cc1</smiles>

Standard conditions: $\mathrm{n}=0.5 \mathrm{mmol}, \mathrm{c}=0.2 \mathrm{M}, \mathrm{V}=2.5 \mathrm{~mL}$, inert atmosphere, $\mathrm{T}=25^{\circ} \mathrm{C}, \mathrm{I}=10$ W

Stock solution: $\mathrm{n}=0.5 \mathrm{mmol}, \mathrm{c}=0.222 \mathrm{M}, \mathrm{V}=2.25 \mathrm{~mL}$, $\mathrm{p}$-anisaldehyde: $68 \mathrm{mg}$, tert-butyl cinnamyl carbonate: $351 \mathrm{mg}, \mathrm{Ir}-1: 4.57 \mathrm{mg}, \mathrm{CoSO}_{4} \bullet \mathrm{H}_{2} \mathrm{O}: 8.65 \mathrm{mg}, \mathrm{L}_{3}: 13.42 \mathrm{mg}$, DIPEA: 100 $\mu L$, DMF: $2.25 \mathrm{~mL}$

Stock solution 'big scale': $\mathrm{n}=7.34 \mathrm{mmol}, \mathrm{c}=0.2 \mathrm{M}, \mathrm{V}=36.7 \mathrm{~mL}, \mathrm{p}$-anisaldehyde: $1.0 \mathrm{~g}$, tert-butyl cinnamyl carbonate: $5.16 \mathrm{~g}$, Ir-1: $67 \mathrm{mg}, \mathrm{CoSO}_{4} \bullet \mathrm{H}_{2} \mathrm{O}: 127 \mathrm{mg}, \mathrm{L}_{3}: 197 \mathrm{mg}$, DIPEA: $3.64 \mathrm{~mL}$, DMF: $36.7 \mathrm{~mL}$

Table S8 Preparation of sensitivity Assessment of Reaction p-anisaldehyde with tert-butyl

\begin{tabular}{|c|c|c|}
\hline Number & Experiment & Preparation \\
\hline 1 & High c & $2.25 \mathrm{~mL}$ stock sol. \\
\hline 2 & Low c & $2.25 \mathrm{~mL}$ stock sol. + $0.5 \mathrm{~mL}$ DMF \\
\hline 3 & High $\mathrm{H}_{2} \mathrm{O}$ & $2.25 \mathrm{~mL}$ stock sol. $+0.25 \mathrm{~mL} \mathrm{DMF}+25 \mu \mathrm{L} \mathrm{H} \mathrm{H}_{2} \mathrm{O}$ \\
\hline 4 & Low $\mathrm{O}_{2}$ & $2.25 \mathrm{~mL}$ stock sol. + $0.25 \mathrm{~mL}$ DMF + degas \\
\hline 5 & High $\mathrm{O}_{2}$ & $2.25 \mathrm{~mL}$ stock sol. $+0.25 \mathrm{~mL} \mathrm{DMF}+25 \mathrm{~mL}$ air \\
\hline 6 & Low T & $2.25 \mathrm{~mL}$ stock sol. $+0.25 \mathrm{~mL} \mathrm{DMF}, \mathrm{T}=15^{\circ} \mathrm{C}$ \\
\hline 7 & High T & $2.25 \mathrm{~mL}$ stock sol. $+0.25 \mathrm{~mL}$ DMF, $\mathrm{T}=35^{\circ} \mathrm{C}$ \\
\hline 8 & Low I & $2.25 \mathrm{~mL}$ stock sol. $+0.25 \mathrm{~mL}$ DMF, I = $1 \mathrm{~W}$ \\
\hline 9 & High I & $2.25 \mathrm{~mL}$ stock sol. $+0.25 \mathrm{~mL}$ DMF, I $=10 \mathrm{~W}$ \\
\hline 10 & Control & $2.25 \mathrm{~mL}$ stock sol. + $0.25 \mathrm{~mL}$ DMF \\
\hline 11 & Big scale & 36.7 mL stock solution 'big scale' \\
\hline
\end{tabular}
cinnamyl carbonate.

Table S9 Results of the Sensitivity Assessment of Reaction p-anisaldehyde with tert-butyl cinnamyl carbonate.

\begin{tabular}{|c|c|c|c|c|c|}
\hline Number & Experiment & Yield 1/\% & Yield 2/\% & Average Y/\% & Deviation/\% \\
\hline 1 & High c & 86 & 88 & 87 & -2 \\
\hline 2 & Low C & 88 & 84 & 86 & -3 \\
\hline 3 & High $\mathrm{H}_{2} \mathrm{O}$ & 13 & 15 & 14 & -75 \\
\hline 4 & Low O $_{2}$ & 83 & 91 & 87 & -2 \\
\hline
\end{tabular}




\begin{tabular}{|c|c|c|c|c|c|}
\hline 5 & High $\mathrm{O}_{2}$ & 0 & 0 & 0 & -89 \\
\hline 6 & Low T & 36 & 44 & 40 & -49 \\
\hline 7 & High T & 88 & 82 & 85 & -4 \\
\hline 8 & Low I & 37 & 35 & 36 & -53 \\
\hline 9 & High I & 89 & 87 & 88 & -1 \\
\hline 10 & Control & 88 & 90 & 89 & \\
\hline 11 & Big scale & 81 & 85 & 83 & -6 \\
\hline
\end{tabular}

Radar diagram:

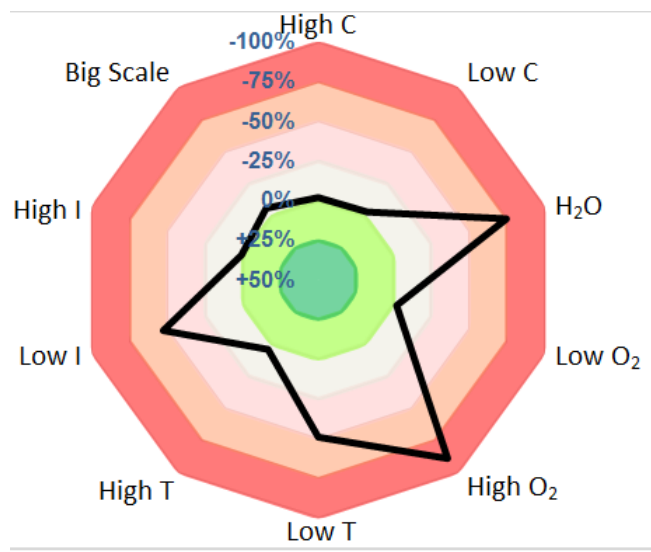

Figure S3. Condition-based Sensitivity Screen 


\section{S8 Mechanism Studies}

Step 1: Firstly, $\mathrm{CoBr}_{2}$ (10 mol\%), $\mathbf{L}_{3}(10 \mathrm{~mol} \%)$, and Ir-1 (1 mol\%) were added to a solution of DIPEA in DMF ( $5 \mathrm{~mL}, 0.2 \mathrm{M})$ resulting in a green mixture.

Step 2: The reaction mixture was stirred at room temperature under irradiation of $10 \mathrm{~W} 450 \mathrm{~nm}$ LED. After 10h, the mixture was turned to blue indicating the formation of the Co' 7 species.

Step 3: Then, the allyl acetate (3 eq.) was added. The reaction mixture was stirred at room temperature without light for $3 \mathrm{~h}$. The mixture was turned to brown. It was speculated that the $\pi$ - allylcobalt"II 8 species was generated via the oxidative addition of allyl acetate to a low-valent Co' complex 7.

To this reaction mixture was added $1 \mathrm{a}$ ( $1 \mathrm{mmol}, 1$ eq.) and stirred in dark. After $20 \mathrm{~h}$, the color of the reaction mixture did not change and no product $2 \mathbf{a}$ was observed. This result indicated that $\pi$ - allylcobalt"l 8 species could not react with aldehyde.

Step 4: For the same time, to another reaction mixture (same as above) was added 1a (1 mmol, 1 eq.). The mixture was stirred at room temperature with irradiation of $10 \mathrm{~W} 450 \mathrm{~nm}$ LED for $20 \mathrm{~h}$. The ${ }^{1} \mathrm{H}$ NMR analysis of the crude product indicated that the yield of $\mathbf{2 a}$ was $50 \%$.

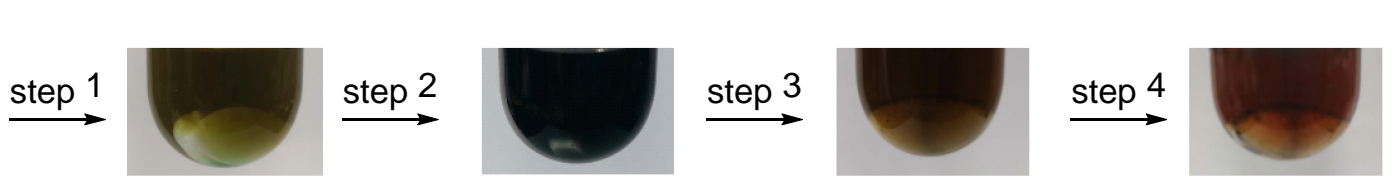<smiles></smiles>

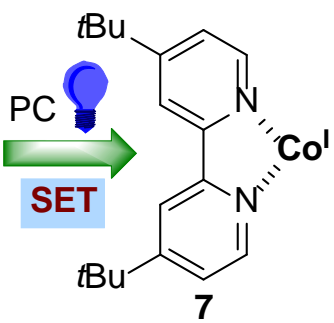

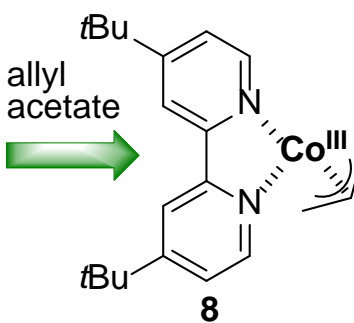

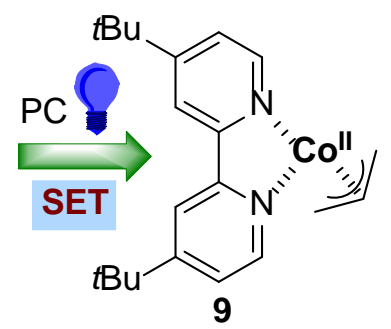

$1 \mathrm{a}$<smiles>COc1ccc(C=O)cc1</smiles>

$1 \mathbf{a}$<smiles>C=CCC(O)c1ccc(OC)cc1</smiles>

$2 a$ 


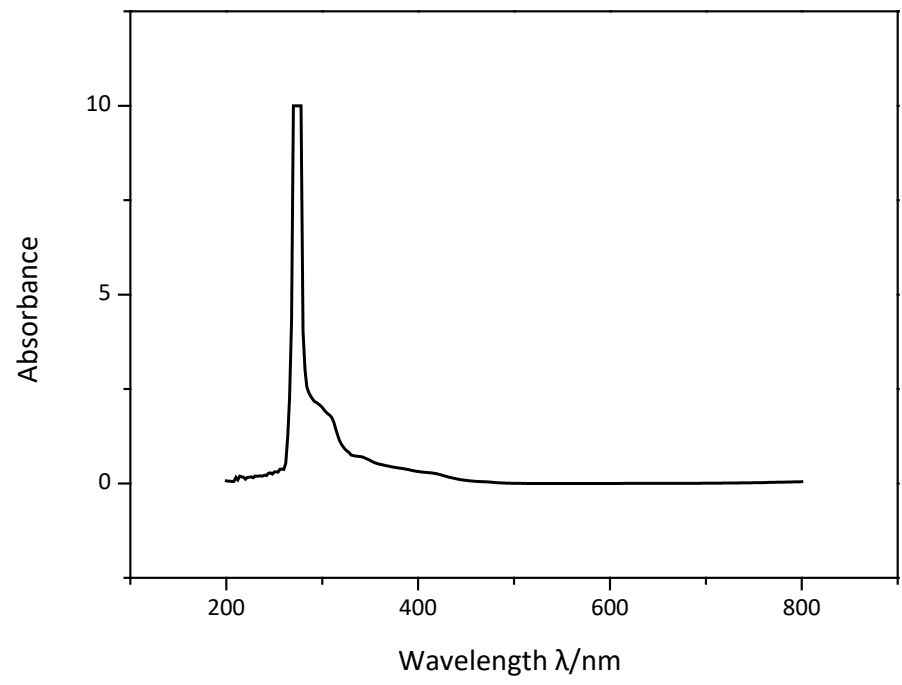

Figure S4. Uv-vis spectrum of Ir-1 (0.2 mM) in DMF

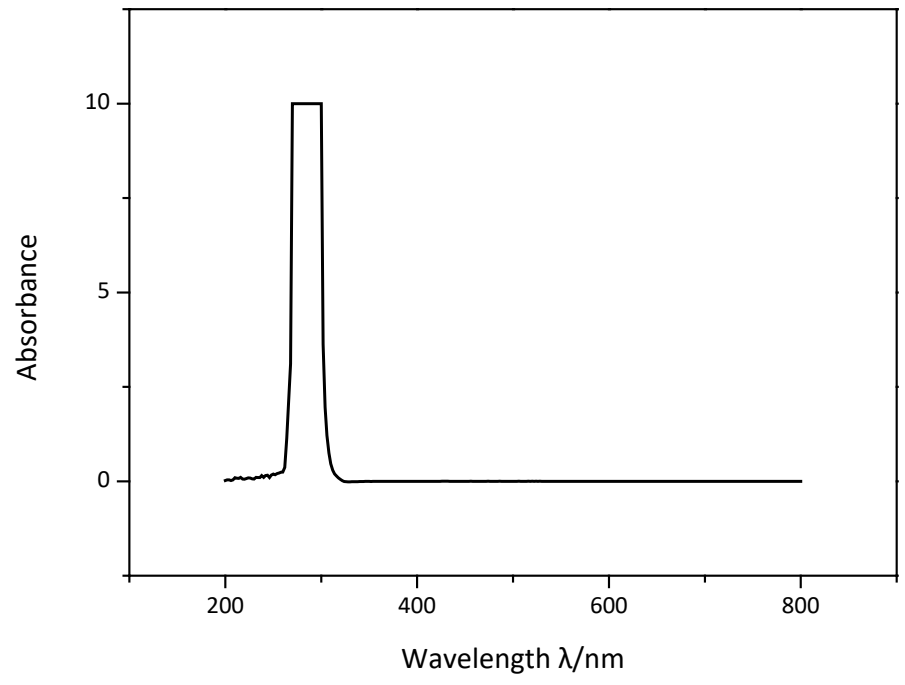

Figure S5. Uv-vis spectrum of $L_{3}(2 \mathrm{mM})$ in DMF 


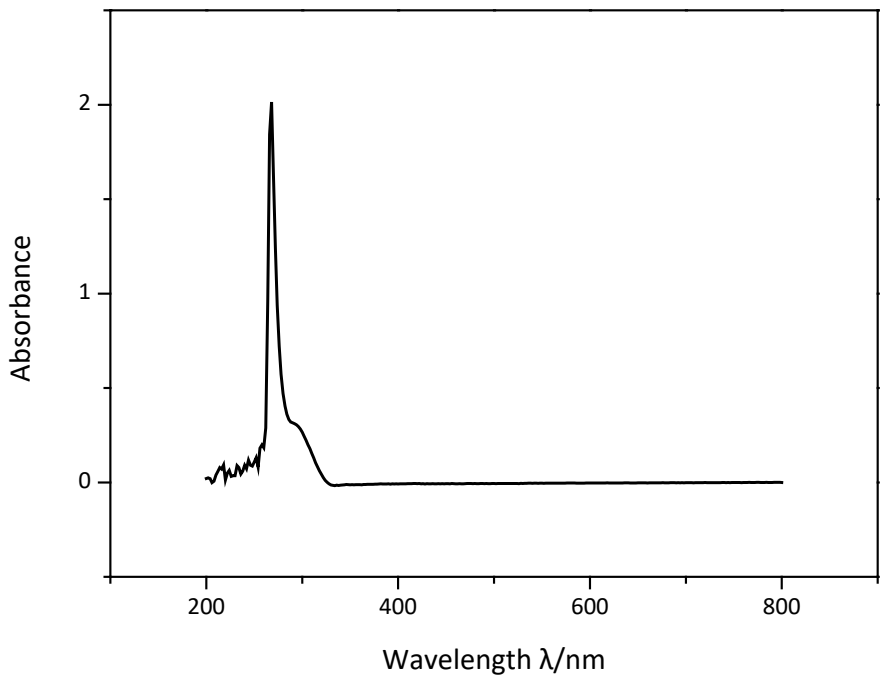

Figure S6. Uv-vis spectrum of DIPEA (0.06 M) in DMF

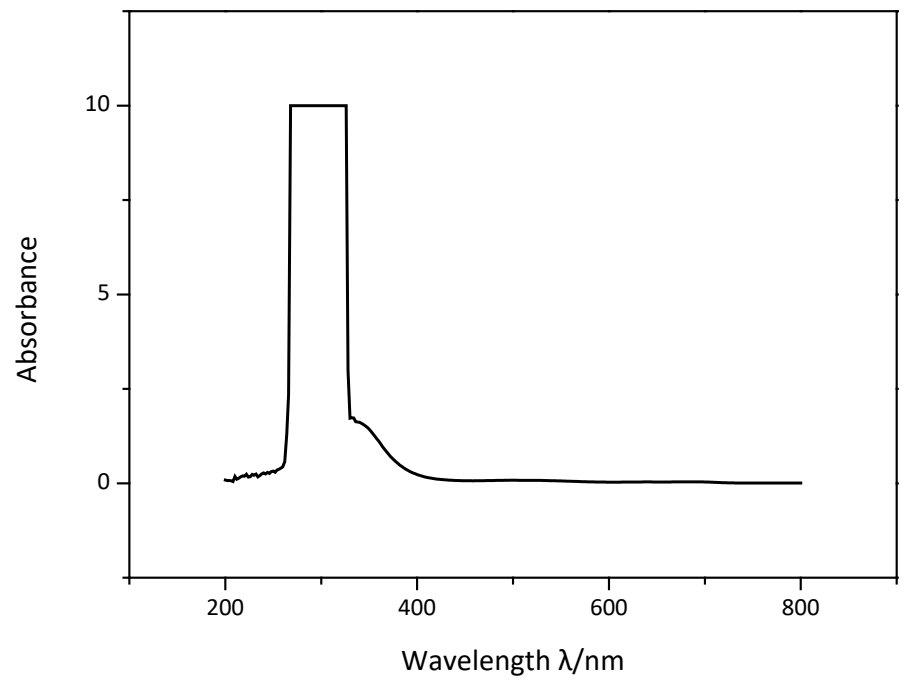

Figure S7. Uv-vis spectrum of $\mathrm{CoBr}_{2}(2 \mathrm{mM})+\mathrm{L}_{3}(2 \mathrm{mM})$ in DMF 


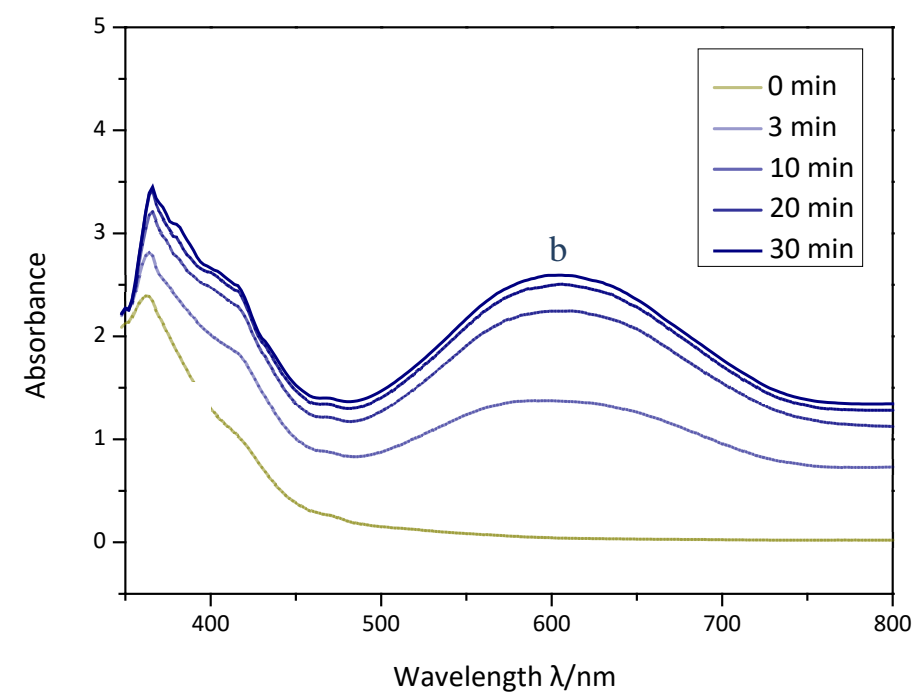

Figure S8. Uv-vis spectra for the generation of $\mathrm{Co}(\mathrm{I})$ from $\mathrm{Co}(\mathrm{II})$

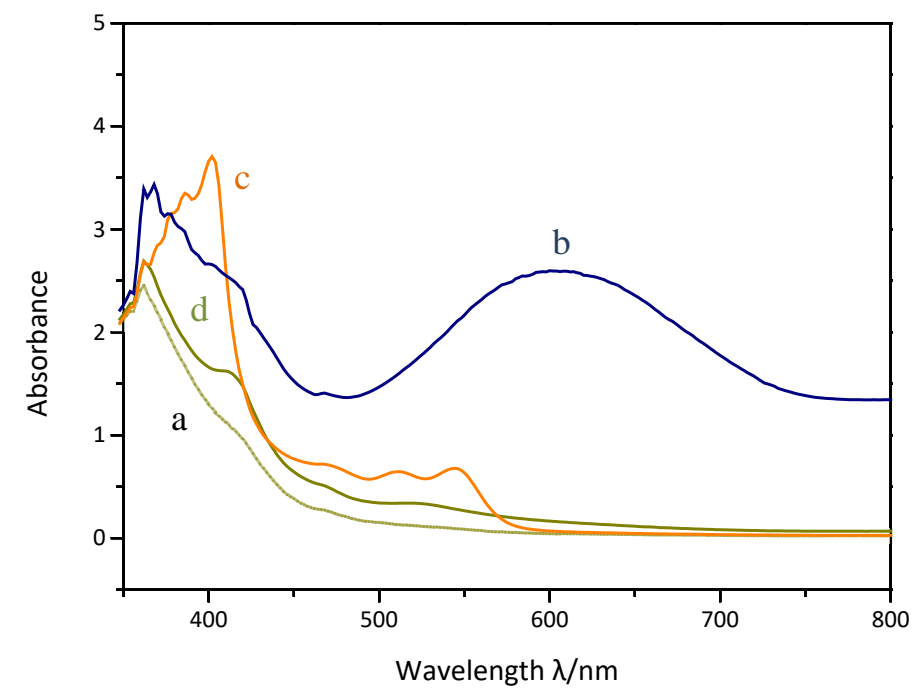

Figure S9. Uv-vis spectra of proposed a Co", b Co', c allyl-Co"', d allyl-Co"

All spectra are zeroed with respect to a straight-DMF blank. Under $\mathrm{N}_{2}$, the Ir-1 (4.57 mg, 1 mol\%), $\mathrm{CoBr}_{2}$ (10.9 mg, $\left.10 \mathrm{~mol} \%\right)$, and DIPEA (0.25 mL, 3 eq.) were added into DMF (25 mL) and $3 \mathrm{~mL}$ solution was injected into a cuvette. Ultraviolet-visible spectroscopy was used to monitor the redox changes of cobalt in the reaction under the standard conditions. Figure S8 shows the photoreduction of $\mathrm{Co}^{\prime \prime}$ and the generation of $\mathrm{Co}^{\prime}$ (absorption band $\mathrm{b}$ ). Then the allyl acetate was added into the reaction mixture in the dark and Uv-vis spectrum was collected as shown in Figure S9, absorption band C. Subsequently, exposing the reaction mixture to irradiating and Uv-vis spectrum was collected as shown in Figure S9, absorption band d. 
<smiles>C=CCC(O)c1ccc(OC)cc1</smiles>

$2 a$

2a, $\mathrm{Rf}=0.5$ (PE:EA = 3:1), column solvent: $\mathrm{PE}: \mathrm{EtOAC}=20: 1.80 \%$, colorless oil, ${ }^{1} \mathrm{H}$ NMR $(400$ $\left.\mathrm{MHz}, \mathrm{CDCl}_{3}\right) \delta 7.28(\mathrm{~d}, \mathrm{~J}=8.8 \mathrm{~Hz}, 2 \mathrm{H}), 6.88(\mathrm{~d}, \mathrm{~J}=8.6 \mathrm{~Hz}, 2 \mathrm{H}), 5.90-5.62(\mathrm{~m}, 1 \mathrm{H}), 5.23-$ $5.07(\mathrm{~m}, 2 \mathrm{H}), 4.68(\mathrm{t}, \mathrm{J}=6.5 \mathrm{~Hz}, 1 \mathrm{H}), 3.80(\mathrm{~s}, 3 \mathrm{H}), 2.53-2.46(\mathrm{~m}, 2 \mathrm{H}), 2.05(\mathrm{~s}, 1 \mathrm{H}) .{ }^{13} \mathrm{C} N M R$ $\left(101 \mathrm{MHz}, \mathrm{CDCl}_{3}\right) \delta 158.98,136.02,134.58,127.04,118.16,113.75,72.95,55.24,43.71$. Organic Letters, 16(16), $4190-4193 ; 2014 .{ }^{1} \mathrm{H}$ NMR $\left(400 \mathrm{MHz}, \mathrm{CDCl}_{3}\right) \delta 7.30-7.26(\mathrm{~m}, 2 \mathrm{H})$, $6.90-6.86(\mathrm{~m}, 2 \mathrm{H}), 5.85-5.74(\mathrm{~m}, 1 \mathrm{H}), 5.18-5.10(\mathrm{~m}, 2 \mathrm{H}), 4.68(\mathrm{t}, \mathrm{J}=6.5 \mathrm{~Hz}, 1 \mathrm{H}), 3.80(\mathrm{~s}$, $3 \mathrm{H}), 2.52-2.47(\mathrm{~m}, 2 \mathrm{H}), 2.04(\mathrm{br}, \mathrm{s}, 1 \mathrm{H}) .{ }^{13} \mathrm{C} \mathrm{NMR}\left(100 \mathrm{MHz}, \mathrm{CDCl}_{3}\right) \delta$ 159.0, 136.1, 134.6, $127.1,118.2,113.8,73.0,55.3,43.7$.<smiles>C=CCC(O)c1ccccc1</smiles>

2b

2b, $\mathrm{Rf}=0.5$ (PE:EA = 3:1), column solvent: PE:EtOAc $=20: 1.68 \%$, colorless oil, ${ }^{1} \mathrm{H}$ NMR $(400$ $\mathrm{MHz}, \mathrm{CDCl}_{3}$ ) $\delta 7.40-7.24$ (m, 5H), 5.81 (ddt, $\left.\mathrm{J}=17.2,10.2,7.1 \mathrm{~Hz}, 1 \mathrm{H}\right), 5.22-5.09(\mathrm{~m}, 2 \mathrm{H})$, $4.73(\mathrm{dd}, \mathrm{J}=7.5,5.5 \mathrm{~Hz}, 1 \mathrm{H}), 2.58-2.45(\mathrm{~m}, 2 \mathrm{H}), 2.15(\mathrm{~s}, 1 \mathrm{H}) .{ }^{13} \mathrm{C} \mathrm{NMR}\left(101 \mathrm{MHz}, \mathrm{CDCl}_{3}\right) \delta$ 143.83, 134.42, 128.36, 127.48, 125.77, 118.31, 73.26, 43.75. Organic Letters, 21(10), 3834 3837; 2019. ${ }^{1} \mathrm{H}$ NMR $\left(\mathrm{CDCl}_{3}\right) \delta 7.38-7.34(\mathrm{~m}, 4 \mathrm{H}), 7.31-7.28(\mathrm{~m}, 1 \mathrm{H}), 5.86-5.78(\mathrm{~m}, 1 \mathrm{H})$, $5.20-5.14(\mathrm{~m}, 2 \mathrm{H}), 4.76-4.73(\mathrm{~m}, 1 \mathrm{H}), 2.57-2.46(\mathrm{~m}, 2 \mathrm{H}), 2.05(\mathrm{~d}, \mathrm{~J}=3.5 \mathrm{~Hz}, 1 \mathrm{H}) .{ }^{13} \mathrm{C}$ $\operatorname{NMR}\left(\mathrm{CDCl}_{3}\right) \delta 143.8,134.4,128.4,127.5,125.8,118.5,73.2,43.9$.<smiles>C=CCC(O)c1ccc(C)cc1</smiles>

2c

2c, $\mathrm{Rf}=0.5$ (PE:EA = 3:1), column solvent: $\mathrm{PE}:$ EtOAc $=20: 1.81 \%$, colorless oil, ${ }^{1} \mathrm{H}$ NMR $(400$ $\left.\mathrm{MHz}, \mathrm{CDCl}_{3}\right) \delta 7.25$ (d, $J=7.9 \mathrm{~Hz}, 2 \mathrm{H}$ ), $7.16(\mathrm{~d}, J=7.9 \mathrm{~Hz}, 2 \mathrm{H}$ ), 5.81 (ddt, $J=17.2,10.2,7.1$ $\mathrm{Hz}, 1 \mathrm{H}$ ), 5.15 (dd, $\mathrm{J}=12.2,11.1 \mathrm{~Hz}, 2 \mathrm{H}), 4.70(\mathrm{t}, \mathrm{J}=6.5 \mathrm{~Hz}, 1 \mathrm{H}), 2.57-2.42(\mathrm{~m}, 2 \mathrm{H}), 2.35(\mathrm{~s}$, 3H), 1.95 (s, 1H). $\left.{ }^{13} \mathrm{C} \mathrm{NMR} \mathrm{(101} \mathrm{MHz,} \mathrm{CDCl}_{3}\right) \delta$ 140.89, 137.16, 134.58, 129.05, 125.74, 118.18, 73.17, 43.71, 21.07. Organic \& Biomolecular Chemistry, 11, $8387-8394 ; 2013 .{ }^{1} \mathrm{H}$ NMR (400 MHz, $\left.\mathrm{CDCl}_{3}\right): \delta 7.24$ (d, J = 8.0, 2H), $7.15(\mathrm{~d}, \mathrm{~J}=8.0,2 \mathrm{H}), 5.75-5.86(\mathrm{~m}, 1 \mathrm{H}), 5.12$ $-5.18(\mathrm{~m}, 2 \mathrm{H}), 4.70$ (brs., $1 \mathrm{H}), 2.48-2.52(\mathrm{~m}, 2 \mathrm{H}), 2.34$ (s, 3H), 1.99 (brs., $1 \mathrm{H}) .{ }^{13} \mathrm{C}$ NMR $(100$ $\left.\mathrm{MHz}, \mathrm{CDCl}_{3}\right): \delta 141.0,137.2,134.6,129.1,125.8,118.1,73.2,43.8,21.1$. 
<smiles>C=CCC(O)c1ccccc1-c1ccccc1</smiles>

2d

2d, $\mathrm{Rf}=0.6$ (PE:EA = 5:1), column solvent: PE:EtOAc $=30: 1.74 \%$, yellow oil, ${ }^{1} \mathrm{H}$ NMR $(400$ $\left.\mathrm{MHz}, \mathrm{CDCl}_{3}\right) \delta 7.65(\mathrm{dd}, \mathrm{J}=7.8,1.1 \mathrm{~Hz}, 1 \mathrm{H}), 7.47-7.29(\mathrm{~m}, 7 \mathrm{H}), 7.23(\mathrm{dd}, \mathrm{J}=7.6,1.3 \mathrm{~Hz}$, $1 \mathrm{H}), 5.78-5.60(\mathrm{~m}, 1 \mathrm{H}), 5.14-5.00(\mathrm{~m}, 2 \mathrm{H}), 4.90-4.78(\mathrm{~m}, 1 \mathrm{H}), 2.52-2.31(\mathrm{~m}, 2 \mathrm{H}), 1.98(\mathrm{~s}$, 1H). ${ }^{13} \mathrm{C}$ NMR $\left(101 \mathrm{MHz}, \mathrm{CDCl}_{3}\right) \delta 141.06,140.86,140.69,134.67,129.98,129.28,128.14$, $127.82,127.19,127.09,125.80,118.19,69.34,43.28 . \mathrm{HRMS}^{\mathrm{p} E S I}(\mathrm{~m} / \mathrm{z})[\mathrm{M}-\mathrm{OH}]^{+}$calculated for $\mathrm{C}_{16} \mathrm{H}_{17}, 207.1168$, found 207.1167.<smiles>C=CCC(O)c1c(C)cc(C)cc1C</smiles>

2e, $\mathrm{Rf}=0.5$ (PE:EA = 3:1), column solvent: PE:EtOAc $=20: 1.90 \%$, colorless oil, ${ }^{1} \mathrm{H}$ NMR $(400$ $\left.\mathrm{MHz}, \mathrm{CDCl}_{3}\right) \delta 6.85(\mathrm{~s}, 2 \mathrm{H}), 5.99-5.78(\mathrm{~m}, 1 \mathrm{H}), 5.29-5.10(\mathrm{~m}, 3 \mathrm{H}), 2.84-2.65(\mathrm{~m}, 2 \mathrm{H}), 2.43$ (s, 6H), $2.28(\mathrm{~s}, 3 \mathrm{H}), 1.99(\mathrm{~s}, 1 \mathrm{H}) .{ }^{13} \mathrm{C}$ NMR $\left(101 \mathrm{MHz}, \mathrm{CDCl}_{3}\right) \delta$ 136.49, 135.92, 135.92, 135.24, 130.08, 117.60, 70.65, 40.27, 20.69, 20.66. Organic \& Biomolecular Chemistry, 11(48), 8387 - 8394; 2013. ${ }^{1} \mathrm{H}$ NMR (400 MHz, $\left.\mathrm{CDCl}_{3}\right): \delta 6.81$ (s, 2H), $5.78-5.89(\mathrm{~m}, 1 \mathrm{H}), 5.10-$ $5.19(\mathrm{~m}, 3 \mathrm{H}), 2.66-2.74(\mathrm{~m}, 1 \mathrm{H}), 2.46-2.50(\mathrm{~m}, 1 \mathrm{H}), 2.40(\mathrm{~s}, 6 \mathrm{H}), 2.24(\mathrm{~s}, 3 \mathrm{H}), 1.86$ (brs., 1H). ${ }^{13} \mathrm{C}$ NMR $\left(100 \mathrm{MHz}, \mathrm{CDCl}_{3}\right)$ : $\delta 136.6,136.0,135.9,135.3,130.1,117.7,70.7,40.3,20.73$, 20.7 .<smiles>C=CCC(O)c1ccccc1C</smiles>

\section{$2 f$}

2f, $\mathrm{Rf}=0.5$ (PE:EA = 3:1), column solvent: PE:EtOAc $=20: 1.69 \%$, colorless oil, ${ }^{1} \mathrm{H}$ NMR $(400$ $\left.\mathrm{MHz}, \mathrm{CDCl}_{3}\right) \delta 7.51(\mathrm{~d}, \mathrm{~J}=7.5 \mathrm{~Hz}, 1 \mathrm{H}), 7.33-7.05(\mathrm{~m}, 3 \mathrm{H}), 5.98-5.71(\mathrm{~m}, 1 \mathrm{H}), 5.29-5.12$ $(\mathrm{m}, 2 \mathrm{H}), 4.99(\mathrm{dd}, \mathrm{J}=8.2,4.5 \mathrm{~Hz}, 1 \mathrm{H}), 2.62-2.42(\mathrm{~m}, 2 \mathrm{H}), 2.37(\mathrm{~s}, 3 \mathrm{H}), 2.05(\mathrm{~s}, 1 \mathrm{H}) .{ }^{13} \mathrm{C} N M R$ $\left(101 \mathrm{MHz}, \mathrm{CDCl}_{3}\right) \delta 141.91,134.70,134.31,130.31,127.19,126.21,125.14,118.22,69.64$, 42.58, 19.01. Tetrahedron Letters, 58(26), 2592 - 2595; 2017. ${ }^{1} \mathrm{H} \mathrm{NMR}\left(400 \mathrm{MHz}, \mathrm{CDCl}_{3}\right.$, TMS): $\delta 7.49$ (d, J = 8.0 Hz, 1H), $7.23(\mathrm{t}, \mathrm{J}=7.2 \mathrm{~Hz}, 1 \mathrm{H}), 7.17$ (td, J = 7.2, $1.2 \mathrm{~Hz}, 1 \mathrm{H}), 7.14(\mathrm{~d}$, $\mathrm{J}=6.4 \mathrm{~Hz}, 1 \mathrm{H}), 5.90-5.80(\mathrm{~m}, 1 \mathrm{H}), 5.20-5.14(\mathrm{~m}, 2 \mathrm{H}), 4.98(\mathrm{q}, \mathrm{J}=4.8 \mathrm{~Hz}, 1 \mathrm{H}), 2.53-2.38$ (m, 2H), 2.33 (s, 3H), 2.05 (s, br, 1H). ${ }^{13} \mathrm{C}$ NMR (100 MHz, CDCl 3 , TMS): $\delta$ 142.06, 134.85, $134.43,130.42,127.31,126.34,125.28,118.31,69.74,42.71,19.15$. 
<smiles>C=CCC(O)c1ccc2c(c1)OCO2</smiles>

$2 \mathrm{~g}$

2g, Rf $=0.4$ (PE:EA = 5:1), column solvent: PE:EtOAc $=20: 1.58 \%$, colorless oil, ${ }^{1} \mathrm{H}$ NMR $(400$ $\left.\mathrm{MHz}, \mathrm{CDCl}_{3}\right) \delta 6.85(\mathrm{~d}, \mathrm{~J}=1.5 \mathrm{~Hz}, 1 \mathrm{H}), 6.83-6.70(\mathrm{~m}, 2 \mathrm{H}), 5.93(\mathrm{~s}, 2 \mathrm{H}), 5.77(\mathrm{~d}, J=7.0 \mathrm{~Hz}$, $1 \mathrm{H}), 5.22-5.03(\mathrm{~m}, 2 \mathrm{H}), 4.62(\mathrm{~d}, J=2.7 \mathrm{~Hz}, 1 \mathrm{H}), 2.45(\mathrm{t}, \mathrm{J}=6.8 \mathrm{~Hz}, 2 \mathrm{H}), 2.21(\mathrm{~d}, J=2.9 \mathrm{~Hz}$, 1H). ${ }^{13} \mathrm{C}$ NMR $\left(101 \mathrm{MHz}, \mathrm{CDCl}_{3}\right) \delta 147.63,146.80,137.94,134.38,119.15,118.21,107.96$, 106.32, 100.90, 73.15, 43.72. Chemical Communications, 55(48), $6838-6841 ; 2019 .{ }^{1} \mathrm{H}$ NMR $\left(400 \mathrm{MHz}, \mathrm{CDCl}_{3}, 25^{\circ} \mathrm{C}\right): \delta 6.85(\mathrm{~d}, \mathrm{~J}=1.5 \mathrm{~Hz}, 1 \mathrm{H}), 6.80-6.72(\mathrm{~m}, 2 \mathrm{H}), 5.92(\mathrm{~s}, 2 \mathrm{H}), 5.82-$ $5.70(\mathrm{~m}, 1 \mathrm{H}), 5.16-5.09(\mathrm{~m}, 2 \mathrm{H}), 4.62(\mathrm{t}, \mathrm{J}=6.5 \mathrm{~Hz}, 1 \mathrm{H}), 2.48-2.42(\mathrm{~m}, 2 \mathrm{H}) .{ }^{13} \mathrm{C}$ NMR $(100$ $\left.\mathrm{MHz}, \mathrm{CDCl}_{3}, 25^{\circ} \mathrm{C}\right): \delta 147.7,146.8,138.0,134.4,119.2,118.3,108.0,106.4,100.9,73.2$, 43.8 .<smiles>C=CCC(O)c1ccc(OC)c(OC)c1</smiles>

$2 \mathrm{~h}$

2h, $\mathrm{Rf}=0.5$ (PE:EA = 2:1), column solvent: $\mathrm{PE}: \mathrm{EtOAc}=10: 1.51 \%$, colorless oil, ${ }^{1} \mathrm{H}$ NMR $(400$ $\left.\mathrm{MHz}, \mathrm{CDCl}_{3}\right) \delta 6.92(\mathrm{~d}, \mathrm{~J}=1.8 \mathrm{~Hz}, 1 \mathrm{H}), 6.90-6.85(\mathrm{~m}, 1 \mathrm{H}), 6.83(\mathrm{~d}, \mathrm{~J}=8.2 \mathrm{~Hz}, 1 \mathrm{H}), 5.90-$ $5.70(\mathrm{~m}, 1 \mathrm{H}), 5.22-5.07(\mathrm{~m}, 2 \mathrm{H}), 4.68(\mathrm{t}, \mathrm{J}=6.5 \mathrm{~Hz}, 1 \mathrm{H}), 3.89(\mathrm{~s}, 3 \mathrm{H}), 3.87(\mathrm{~s}, 3 \mathrm{H}), 2.50(\mathrm{t}, \mathrm{J}$ $=7.1 \mathrm{~Hz}, 2 \mathrm{H}), 2.05(\mathrm{~s}, 1 \mathrm{H}) \cdot{ }^{13} \mathrm{C}$ NMR $\left(101 \mathrm{MHz}, \mathrm{CDCl}_{3}\right) \delta 148.97,148.36,136.53,134.54$, 118.27, 118.02, 110.87, 108.90, 73.15, 55.88, 55.82, 43.79. HRMS+pESI (m/z) $[\mathrm{M}-\mathrm{OH}]^{+}$ calculated for $\mathrm{C}_{12} \mathrm{H}_{15} \mathrm{O}_{2}, 191.1067$, found 191.1067.<smiles>C=CCC(O)c1ccc(OC)c(OC)c1OC</smiles>

2i

2i, $\mathrm{Rf}=0.6(\mathrm{PE}: \mathrm{EA}=2: 1)$, column solvent: $\mathrm{PE}: \mathrm{EtOAc}=10: 1.53 \%$, colorless oil, ${ }^{1} \mathrm{H}$ NMR $(400$ $\left.\mathrm{MHz}, \mathrm{CDCl}_{3}\right) \delta 7.02(\mathrm{~d}, \mathrm{~J}=8.6 \mathrm{~Hz}, 1 \mathrm{H}), 6.65(\mathrm{~d}, \mathrm{~J}=8.6 \mathrm{~Hz}, 1 \mathrm{H}), 5.93-5.75(\mathrm{~m}, 1 \mathrm{H}), 5.19-$ $5.05(\mathrm{~m}, 2 \mathrm{H}), 4.95-4.85(\mathrm{~m}, 1 \mathrm{H}), 3.93(\mathrm{~s}, 3 \mathrm{H}), 3.84(\mathrm{~s}, 6 \mathrm{H}), 2.50(\mathrm{t}, \mathrm{J}=6.9 \mathrm{~Hz}, 2 \mathrm{H}), 2.45(\mathrm{~s}$, 1H). ${ }^{13} \mathrm{C}$ NMR $\left(101 \mathrm{MHz}, \mathrm{CDCl}_{3}\right) \delta 152.99,150.95,141.80,135.00,129.37,121.05,117.70$, 107.04, 69.00, 61.08, 60.65, 55.91, 42.70. Organic Letters, 16(16), $4190-4193 ; 2014 .{ }^{1} \mathrm{H}$ $\operatorname{NMR}\left(400 \mathrm{MHz}, \mathrm{CDCl}_{3}\right) \delta 7.03(\mathrm{~d}, \mathrm{~J}=8.6 \mathrm{~Hz}, 1 \mathrm{H}), 6.66(\mathrm{~d}, \mathrm{~J}=8.6 \mathrm{~Hz}, 1 \mathrm{H}), 5.90-5.79(\mathrm{~m}, 1 \mathrm{H})$ $5.17-5.09(\mathrm{~m}, 2 \mathrm{H}), 4.90(\mathrm{dd}, \mathrm{J}=7.6,5.5 \mathrm{~Hz}, 2 \mathrm{H}), 3.93(\mathrm{~s}, 3 \mathrm{H}), 3.86(\mathrm{~s}, 3 \mathrm{H}), 3.85(\mathrm{~s}, 3 \mathrm{H}), 2.59$ $-2.43(\mathrm{~m}, 3 \mathrm{H}) .{ }^{13} \mathrm{C}$ NMR $\left(100 \mathrm{MHz}, \mathrm{CDCl}_{3}\right) \delta 153.0,150.9,141.8,135.0,129.4,121.0,117.7$, 107.1, 69.0, 61.1, 60.6, 55.9, 42.7. 
<smiles>C=CCC(O)c1ccc(O)cc1</smiles>

2j

2j, $\mathrm{Rf}=0.5$ (PE:EA = 2:1), column solvent: PE:EtOAc $=10: 1.78 \%$, yellow oil, ${ }^{1} \mathrm{H}$ NMR $(400$ $\left.\mathrm{MHz}, \mathrm{CDCl}_{3}\right) \delta 7.22(\mathrm{~d}, \mathrm{~J}=8.5 \mathrm{~Hz}, 2 \mathrm{H}), 6.79(\mathrm{~d}, \mathrm{~J}=8.6 \mathrm{~Hz}, 2 \mathrm{H}), 5.92-5.68(\mathrm{~m}, 1 \mathrm{H}), 5.32(\mathrm{~s}$, $1 \mathrm{H}), 5.21-5.08(\mathrm{~m}, 2 \mathrm{H}), 4.68(\mathrm{t}, J=6.5 \mathrm{~Hz}, 1 \mathrm{H}), 2.50(\mathrm{t}, J=6.8 \mathrm{~Hz}, 2 \mathrm{H}), 2.10(\mathrm{~s}, 1 \mathrm{H}) .{ }^{13} \mathrm{C}$ NMR $\left(101 \mathrm{MHz}, \mathrm{CDCl}_{3}\right) \delta 155.11,135.86,134.47,127.31,118.38,115.26,73.05,43.65$. European Journal of Organic Chemistry, 15, $3123-3130 ; 2013 .{ }^{1} \mathrm{H}$ NMR $(400 \mathrm{MHz}$, $\mathrm{CDCl}_{3}$ ): $\delta 2.41-2.52\left(\mathrm{~m}, 2 \mathrm{H},-\mathrm{CH}_{2}-\right), 2.95$ (br. s, $\left.1 \mathrm{H}, \mathrm{OH}\right), 4.61$ (dd, J = 6.8 Hz, J = 6.4 Hz, $1 \mathrm{H},-\mathrm{CH}-), 5.05-5.11\left(\mathrm{~m}, 2 \mathrm{H},=\mathrm{CH}_{2}\right), 5.66-5.76(\mathrm{~m}, 1 \mathrm{H},=\mathrm{CH}), 6.71(\mathrm{~d}, \mathrm{~J}=8.4 \mathrm{~Hz}, 2 \mathrm{H}, \mathrm{Ar})$, $7.11(\mathrm{~d}, \mathrm{~J}=8.4 \mathrm{~Hz}, 2 \mathrm{H}, \mathrm{Ar}) \mathrm{ppm} .{ }^{13} \mathrm{C}$ NMR $\left(100 \mathrm{MHz}, \mathrm{CDCl}_{3}\right)$ : $\delta$ 153.4, 135.1, 134.4, 127.5, 118.4, 115.5, 73.6, 43.3 ppm.<smiles>C=CCC(O)c1ccc(Br)cc1</smiles>

2k, Rf $=0.5$ (PE:EA = 3:1), column solvent: PE:EtOAc $=20: 1.52 \%$, colorless oil, ${ }^{1} \mathrm{H}$ NMR (400 $\left.\mathrm{MHz}, \mathrm{CDCl}_{3}\right) \delta 7.47(\mathrm{~d}, \mathrm{~J}=8.4 \mathrm{~Hz}, 2 \mathrm{H}), 7.23(\mathrm{~d}, \mathrm{~J}=8.3 \mathrm{~Hz}, 2 \mathrm{H}), 5.85-5.70(\mathrm{~m}, 1 \mathrm{H}), 5.19-$ $5.10(\mathrm{~m}, 2 \mathrm{H}), 4.69(\mathrm{dd}, \mathrm{J}=7.7,5.1 \mathrm{~Hz}, 1 \mathrm{H}), 2.56-2.38(\mathrm{~m}, 2 \mathrm{H}), 2.00(\mathrm{~s}, 1 \mathrm{H}) .{ }^{13} \mathrm{C}$ NMR $(101$ $\left.\mathrm{MHz}, \mathrm{CDCl}_{3}\right) \delta 142.79,133.90,131.44,127.53,121.22,118.82,72.56,43.77$. Organic Letters, 21(10), 3834 - 3837; 2019. ${ }^{1} \mathrm{H}$ NMR $\left(\mathrm{CDCl}_{3}\right) \delta 7.50$ (dd, J = 3.0, $\left.1.5 \mathrm{~Hz}, 1 \mathrm{H}\right), 7.48$ (dd, J = 2.5, $2.0 \mathrm{~Hz}, 1 \mathrm{H}), 7.26-7.25(\mathrm{~m}, 1 \mathrm{H}), 7.25-7.23(\mathrm{~m}, 1 \mathrm{H}), 5.83-5.75(\mathrm{~m}, 1 \mathrm{H}), 5.20-5.18(\mathrm{~m}, 1 \mathrm{H})$, $5.17-5.15(\mathrm{~m}, 1 \mathrm{H}), 4.72(\mathrm{t}, \mathrm{J}=4.0 \mathrm{~Hz}, 1 \mathrm{H}), 2.54-2.42(\mathrm{~m}, 2 \mathrm{H}), 2.11(\mathrm{~s}, 1 \mathrm{H}) .{ }^{13} \mathrm{C} \mathrm{NMR}\left(\mathrm{CDCl}_{3}\right)$ $\delta 142.7,133.9,131.4,127.5,121.2,119.0,72.5,43.9$.<smiles>C=CCC(O)c1ccc(F)cc1</smiles>

2I

2I, $\mathrm{Rf}=0.5(\mathrm{PE}: \mathrm{EA}=3: 1)$, column solvent: $\mathrm{PE}: \mathrm{EtOAC}=20: 1.43 \%$, colorless oil, ${ }^{1} \mathrm{H}$ NMR $(400$ $\left.\mathrm{MHz}, \mathrm{CDCl}_{3}\right) \delta 7.32(\mathrm{dd}, \mathrm{J}=8.6,5.5 \mathrm{~Hz}, 2 \mathrm{H}), 7.03(\mathrm{t}, \mathrm{J}=8.7 \mathrm{~Hz}, 2 \mathrm{H}), 5.88-5.70(\mathrm{~m}, 1 \mathrm{H}), 5.24$ $-5.06(\mathrm{~m}, 2 \mathrm{H}), 4.71(\mathrm{t}, \mathrm{J}=6.4 \mathrm{~Hz}, 1 \mathrm{H}), 2.58-2.38(\mathrm{~m}, 2 \mathrm{H}), 2.13(\mathrm{~s}, 1 \mathrm{H}) .{ }^{13} \mathrm{C} \mathrm{NMR}(101 \mathrm{MHz}$, $\left.\mathrm{CDCl}_{3}\right) \delta 134.13,127.47,127.39,118.65,115.28,115.07,72.61,43.91 . \mathrm{HRMS}+\mathrm{pESI}(\mathrm{m} / \mathrm{z})$ $[\mathrm{M}-\mathrm{OH}]^{+}$calculated for $\mathrm{C}_{10} \mathrm{H}_{10} \mathrm{~F}, 149.30761$, found 149.0759 .<smiles>C=CCC(O)c1ccc2ccccc2c1</smiles>

$2 \mathrm{~m}$ 
2m, Rf $=0.4$ (PE:EA $=3: 1$ ), column solvent: PE:EtOAc $=20: 1.77 \%$, colorless oil, ${ }^{1} \mathrm{H}$ NMR $\left(400 \mathrm{MHz}, \mathrm{CDCl}_{3}\right) \delta 7.91-7.75(\mathrm{~m}, 4 \mathrm{H}), 7.57-7.43(\mathrm{~m}, 3 \mathrm{H}), 5.95-5.76(\mathrm{~m}, 1 \mathrm{H}), 5.27-5.09$ $(\mathrm{m}, 2 \mathrm{H}), 4.90(\mathrm{t}, \mathrm{J}=6.3 \mathrm{~Hz}, 1 \mathrm{H}), 2.68-2.53(\mathrm{~m}, 2 \mathrm{H}), 2.35(\mathrm{~s}, 1 \mathrm{H}) .{ }^{13} \mathrm{C} \mathrm{NMR}\left(101 \mathrm{MHz}, \mathrm{CDCl}_{3}\right)$ $\delta 141.21,134.33,133.23,132.92,128.18,127.93,127.65,126.10,125.78,124.48,123.97$, 118.51, 73.36, 43.70. Chemical Communications, 55(48), $6838-6841 ; 2019 .{ }^{1} \mathrm{H}$ NMR (400 $\left.\mathrm{MHz}, \mathrm{CDCl}_{3}, 25^{\circ} \mathrm{C}\right): \delta 7.85-7.78(\mathrm{~m}, 4 \mathrm{H}), 7.51-7.42(\mathrm{~m}, 3 \mathrm{H}), 5.88-5.77(\mathrm{~m}, 1 \mathrm{H}), 5.21-$ $5.12(\mathrm{~m}, 2 \mathrm{H}), 4.89(\mathrm{dd}, \mathrm{J}=7.2,5.9 \mathrm{~Hz}, 1 \mathrm{H}), 2.66-2.52(\mathrm{~m}, 2 \mathrm{H}) .{ }^{13} \mathrm{C} \mathrm{NMR}\left(100 \mathrm{MHz}, \mathrm{CDCl}_{3}\right.$, $\left.25^{\circ} \mathrm{C}\right)$ : $\delta 141.2,134.3,133.2,132.9,128.2,127.9,127.6,126.1,125.8,124.4,124.0,118.5$, 73.3, 43.7 .<smiles>C=CCC(O)c1ccc(N(C)C)cc1</smiles>

2n

2n, $\mathrm{Rf}=0.4$ (PE:EA = 2:1), column solvent: PE:EtOAc $=10: 1.43 \%$, red oil, ${ }^{1} \mathrm{H}$ NMR $(400 \mathrm{MHz}$, $\left.\mathrm{CDCl}_{3}\right) \delta 7.24(\mathrm{~d}, \mathrm{~J}=8.6 \mathrm{~Hz}, 2 \mathrm{H}), 6.73(\mathrm{~d}, \mathrm{~J}=8.8 \mathrm{~Hz}, 2 \mathrm{H}), 5.92-5.70(\mathrm{~m}, 1 \mathrm{H}), 5.22-5.05(\mathrm{~m}$, 2H), $4.65(\mathrm{~s}, 1 \mathrm{H}), 2.95(\mathrm{~s}, 6 \mathrm{H}), 2.52(\mathrm{~d}, \mathrm{~J}=5.3 \mathrm{~Hz}, 2 \mathrm{H}), 1.87(\mathrm{~s}, 1 \mathrm{H}) .{ }^{13} \mathrm{C}$ NMR $(101 \mathrm{MHz}$, $\left.\mathrm{CDCl}_{3}\right) \delta 150.19,135.01,131.76,126.82,117.79,112.47,73.24,43.47,40.63$. HRMS+pESI $(\mathrm{m} / \mathrm{z})[\mathrm{M}-\mathrm{OH}]^{+}$calculated for $\mathrm{C}_{12} \mathrm{H}_{11} \mathrm{~N}, 174.1277$, found 174.1278 .<smiles>C=CCC(O)c1ccc(Cl)cc1</smiles>

2o, $\mathrm{Rf}=0.5$ (PE:EA = 3:1), column solvent: $\mathrm{PE}: \mathrm{EtOAC}=20: 1.68 \%$, colorless oil, ${ }^{1} \mathrm{H}$ NMR $(400$ $\left.\mathrm{MHz}, \mathrm{CDCl}_{3}\right) \delta 7.31-7.10(\mathrm{~m}, 4 \mathrm{H}), 5.77-5.58(\mathrm{~m}, 1 \mathrm{H}), 5.12-5.00(\mathrm{~m}, 2 \mathrm{H}), 4.61$ (ddd, $\mathrm{J}=7.7$, 5.2, $2.5 \mathrm{~Hz}, 1 \mathrm{H}), 2.46-2.29(\mathrm{~m}, 2 \mathrm{H}), 2.18(\mathrm{~s}, 1 \mathrm{H}) .{ }^{13} \mathrm{C} \mathrm{NMR}\left(101 \mathrm{MHz}, \mathrm{CDCl}_{3}\right) \delta 142.25$, 133.92, 133.07, 128.46, 127.16, 118.73, 72.51, 43.76. Tetrahedron Letters, 60(3), $276-283$; 2019. ${ }^{1} \mathrm{H}$ NMR $\left(\mathrm{CDCl}_{3}, 400 \mathrm{MHz}\right): \delta 7.28-7.22(\mathrm{~m}, 4 \mathrm{H}), 5.78-5.68(\mathrm{~m}, 1 \mathrm{H}), 5.13-5.09(\mathrm{~m}$, $2 \mathrm{H}), 4.67-4.64(\mathrm{~m}, 1 \mathrm{H}), 2.45-2.40(\mathrm{~m}, 2 \mathrm{H}), 2.21-2.20(\mathrm{~m}, 1 \mathrm{H}) .{ }^{13} \mathrm{C} \mathrm{NMR}\left(\mathrm{CDCl}_{3}, 100 \mathrm{MHz}\right)$ : ठ 142.4, 134.1, 133.3, 128.6, 127.3, 118.9, 72.7, 43.9.<smiles>C=CCC(O)c1cccs1</smiles>

$2 p$

2p, Rf $=0.5$ (PE:EA = 3:1), column solvent: PE:EtOAc $=20: 1.54 \%$, colorless oil, ${ }^{1} \mathrm{H}$ NMR $(400$ $\left.\mathrm{MHz}, \mathrm{CDCl}_{3}\right) \delta 7.37-7.27(\mathrm{~m}, 1 \mathrm{H}), 7.09-6.97(\mathrm{~m}, 2 \mathrm{H}), 6.03-5.78(\mathrm{~m}, 1 \mathrm{H}), 5.34-5.19(\mathrm{~m}$, $2 \mathrm{H}), 5.04(\mathrm{t}, J=6.7 \mathrm{~Hz}, 1 \mathrm{H}), 2.77-2.65(\mathrm{~m}, 2 \mathrm{H}), 2.61(\mathrm{~s}, 1 \mathrm{H}) .{ }^{13} \mathrm{C} \mathrm{NMR}\left(101 \mathrm{MHz}, \mathrm{CDCl}_{3}\right) \delta$ 147.76, 133.80, 126.61, 124.55, 123.67, 118.82, 69.34, 43.76. Chemistry A European Journal, 19(41), 13859 - 13864; 2013. ${ }^{1} \mathrm{H}$ NMR (400 MHz, CDCl $)$ ): $\delta 7.25$ (dd, $\mathrm{J}_{1}=5.1 \mathrm{~Hz}, \mathrm{~J}_{2}=1.6 \mathrm{~Hz}$, 1H), $7.00-6.96(\mathrm{~m}, 2 \mathrm{H}), 5.89-5.79(\mathrm{~m}, 1 \mathrm{H}), 5.23-5.15(\mathrm{~m}, 2 \mathrm{H}), 5.01-4.98(\mathrm{~m}, 1 \mathrm{H}), 2.65-$ 
$2.61(\mathrm{~m}, 2 \mathrm{H}), 2.17$ (br s, $1 \mathrm{H}) .{ }^{13} \mathrm{C}$ NMR $\left(100 \mathrm{MHz}, \mathrm{CDCl}_{3}\right): \delta$ 148.0, 134.0, 126.8, 124.7, 123.8, $119.0,69.5,43.9$.<smiles>C=CCC(O)c1ccco1</smiles>

$2 q$

2q, $\mathrm{Rf}=0.5$ (PE:EA = 3:1), column solvent: $\mathrm{PE}: \mathrm{EtOAc}=20: 1.53 \%$, colorless oil, ${ }^{1} \mathrm{H} \mathrm{NMR}(400$ $\left.\mathrm{MHz}, \mathrm{CDCl}_{3}\right) \delta 7.36(\mathrm{~s}, 1 \mathrm{H}), 6.32(\mathrm{~s}, 1 \mathrm{H}), 6.24(\mathrm{~d}, \mathrm{~J}=3.5 \mathrm{~Hz}, 1 \mathrm{H}), 5.89-5.65(\mathrm{~m}, 1 \mathrm{H}), 5.21-$ $5.07(\mathrm{~m}, 2 \mathrm{H}), 4.79-4.64(\mathrm{~m}, 1 \mathrm{H}), 2.71-2.48(\mathrm{~m}, 2 \mathrm{H}), 2.37(\mathrm{~s}, 1 \mathrm{H}) .{ }^{13} \mathrm{C}$ NMR $(101 \mathrm{MHz}$, $\left.\mathrm{CDCl}_{3}\right) \delta 155.98,141.95,133.66,118.54,110.11,106.07,66.90,40.04$. Chemistry A European Journal, 19(41), 13859 - 13864; 2013. ${ }^{1} \mathrm{H}$ NMR $\left(400 \mathrm{MHz}, \mathrm{CDCl}_{3}\right): \delta 7.38$ (dd, $\mathrm{J}_{1}=$ $\left.1.8 \mathrm{~Hz}, \mathrm{~J}_{2}=0.8 \mathrm{~Hz}, 1 \mathrm{H}\right), 6.33\left(\mathrm{dd}, \mathrm{J}_{1}=3.2 \mathrm{~Hz}, \mathrm{~J}_{2}=1.8 \mathrm{~Hz}, 1 \mathrm{H}\right), 6.26-6.25(\mathrm{~m}, 1 \mathrm{H}), 5.87-$ $5.76(\mathrm{~m}, 1 \mathrm{H}), 5.22-5.13(\mathrm{~m}, 2 \mathrm{H}), 4.78-4.73(\mathrm{~m}, 1 \mathrm{H}), 2.69-2.57(\mathrm{~m}, 2 \mathrm{H}), 2.01(\mathrm{~d}, \mathrm{~J}=5.0 \mathrm{~Hz}$, 1H). ${ }^{13} \mathrm{C} \mathrm{NMR}\left(100 \mathrm{MHz}, \mathrm{CDCl}_{3}\right): \delta 156.0,142.0,133.7,118.6,110.1,106.1,66.9,40.1$.<smiles>C=CCC(O)c1ccc(C)o1</smiles>

$2 r$

2r, $\mathrm{Rf}=0.5$ (PE:EA = 5:1), column solvent: PE:EtOAc $=30: 1.64 \%$, colorless oil, ${ }^{1} \mathrm{H}$ NMR $(400$ $\left.\mathrm{MHz}, \mathrm{CDCl}_{3}\right) \delta 6.12(\mathrm{~d}, \mathrm{~J}=3.0 \mathrm{~Hz}, 1 \mathrm{H}), 5.90(\mathrm{~s}, 1 \mathrm{H}), 5.88-5.75(\mathrm{~m}, 1 \mathrm{H}), 5.22-5.11(\mathrm{~m}, 2 \mathrm{H})$, $4.67(\mathrm{q}, \mathrm{J}=6.2 \mathrm{~Hz}, 1 \mathrm{H}), 2.70-2.52(\mathrm{~m}, 2 \mathrm{H}), 2.28(\mathrm{~s}, 3 \mathrm{H}), 2.05(\mathrm{~d}, \mathrm{~J}=4.7 \mathrm{~Hz}, 1 \mathrm{H}) .{ }^{13} \mathrm{C} \mathrm{NMR}$ $\left(101 \mathrm{MHz}, \mathrm{CDCl}_{3}\right) \delta$ 154.12, 151.68, 133.94, 118.27, 106.90, 105.94, 66.87, 39.94, 13.48. Journal of Organic Chemistry, 75(5), $1795-1798 ; 2010 .{ }^{1} \mathrm{H}$ NMR $\left(400 \mathrm{MHz}, \mathrm{CDCl}_{3}\right): 6.09$ (d, J $=3.1 \mathrm{~Hz}, 1 \mathrm{H}), 5.87(\mathrm{~d}, \mathrm{~J}=2.9 \mathrm{~Hz}, 1 \mathrm{H}), 5.79$ (dddd, $\mathrm{J}=17.2,10.2,7.2,6.9 \mathrm{~Hz}, 1 \mathrm{H}), 5.12(\mathrm{~m}$, $2 \mathrm{H}), 4.65(\mathrm{t}, \mathrm{J}=6.8 \mathrm{~Hz}, 1 \mathrm{H}), 2.58(\mathrm{~m}, 2 \mathrm{H}), 2.25(\mathrm{~s}, 3 \mathrm{H}), 2.10(\mathrm{~s}, 1 \mathrm{H}) \cdot{ }^{13} \mathrm{C}$ NMR $(100 \mathrm{MHz}$, $\left.\mathrm{CDCl}_{3}\right): 154.3,151.9,134.2,118.5,107.1,106.2,67.1,40.2,13.7$.<smiles>C=CCC(O)CCN1C(=O)c2ccccc2C1=O</smiles>

2s

2s, $\mathrm{Rf}=0.5$ (PE:EA = 2:1), column solvent: $\mathrm{PE}: \mathrm{EtOAc}=10: 1.31 \%$, colorless oil, ${ }^{1} \mathrm{H}$ NMR $(400$ $\left.\mathrm{MHz}, \mathrm{CDCl}_{3}\right) \delta 7.85(\mathrm{dd}, \mathrm{J}=5.4,3.1 \mathrm{~Hz}, 2 \mathrm{H}), 7.72(\mathrm{dd}, \mathrm{J}=5.5,3.0 \mathrm{~Hz}, 2 \mathrm{H}), 5.88-5.74(\mathrm{~m}$, $1 \mathrm{H}), 5.15-5.02(\mathrm{~m}, 2 \mathrm{H}), 3.87(\mathrm{dd}, J=7.8,5.2 \mathrm{~Hz}, 2 \mathrm{H}), 3.61(\mathrm{dd}, J=6.3,3.6 \mathrm{~Hz}, 1 \mathrm{H}), 2.68(\mathrm{~d}$, $\mathrm{J}=4.3 \mathrm{~Hz}, 1 \mathrm{H}), 2.25(\mathrm{t}, \mathrm{J}=6.9 \mathrm{~Hz}, 2 \mathrm{H}), 1.94-1.65(\mathrm{~m}, 2 \mathrm{H}) .{ }^{13} \mathrm{C} \mathrm{NMR}\left(101 \mathrm{MHz}, \mathrm{CDCl}_{3}\right) \delta$ $168.83,134.53,134.05,132.02,123.33,117.88,67.76,41.62,35.62$, 34.69. Journal of the American Chemical Society, 130(44), $14891-14899$; 2008. ${ }^{1} \mathrm{H}$ NMR $\left(400 \mathrm{MHz}, \mathrm{CDCl}_{3}\right): \delta$ $7.87-7.81(\mathrm{~m}, 2 \mathrm{H}), 7.74-7.69(\mathrm{~m}, 2 \mathrm{H}), 5.86-5.75(\mathrm{~m}, 1 \mathrm{H}), 5.12-5.06(\mathrm{~m}, 2 \mathrm{H}), 3.85(\mathrm{t}, \mathrm{J}=$ $7.6 \mathrm{~Hz}, 2 \mathrm{H}), 3.64-3.59(\mathrm{~m}, 1 \mathrm{H}), 2.71(\mathrm{br}, 1 \mathrm{H}), 2.28(\mathrm{t}, \mathrm{J}=7.2 \mathrm{~Hz}, 2 \mathrm{H}), 1.88-1.66(\mathrm{~m}, 2 \mathrm{H})$. ${ }^{13} \mathrm{C} \mathrm{NMR}\left(100 \mathrm{MHz}, \mathrm{CDCl}_{3}\right): \delta 169.1,134.8,134.3,132.3,123.6,118.1,68.0,41.9,35.9,34.9$. 
<smiles>C=CCC(O)C1CCCCC1</smiles>

2t

2t, $\mathrm{Rf}=0.5$ (PE:EA = 5:1), column solvent: PE:EtOAc $=30: 1.42 \%$, colorless oil, ${ }^{1} \mathrm{H}$ NMR $(400$ $\left.\mathrm{MHz}, \mathrm{CDCl}_{3}\right) \delta 5.98-5.68(\mathrm{~m}, 1 \mathrm{H}), 5.27-5.00(\mathrm{~m}, 2 \mathrm{H}), 3.48-3.26(\mathrm{~m}, 1 \mathrm{H}), 2.39-2.23(\mathrm{~m}$, $1 \mathrm{H}), 2.11(\mathrm{dt}, J=14.9,8.4 \mathrm{~Hz}, 1 \mathrm{H}), 1.93-1.79(\mathrm{~m}, 1 \mathrm{H}), 1.81-1.55(\mathrm{~m}, 5 \mathrm{H}) .1 .45-0.90(\mathrm{~m}$, $6 \mathrm{H}) .{ }^{13} \mathrm{C}$ NMR $\left(101 \mathrm{MHz}, \mathrm{CDCl}_{3}\right) \delta 135.45,117.87,77.32,43.03,38.77,29.04,28.07,26.48$, 26.24, 26.09. Chemical Communications, 55(48), 6838 - 6841; 2019. ${ }^{1} \mathrm{H}$ NMR $(400 \mathrm{MHz}$, $\left.\mathrm{CDCl}_{3}, 25^{\circ} \mathrm{C}\right): \delta 5.82(\mathrm{dddd}, \mathrm{J}=12.5,9.2,8.0,6.4 \mathrm{~Hz}, 1 \mathrm{H}), 5.15-5.09(\mathrm{~m}, 2 \mathrm{H}), 3.39-3.35(\mathrm{~m}$, $1 \mathrm{H}), 2.35-2.28(\mathrm{~m}, 1 \mathrm{H}), 2.11(\mathrm{dt}, \mathrm{J}=14,8.4 \mathrm{~Hz}, 1 \mathrm{H}), 1.84(\mathrm{~d}, \mathrm{~J}=12.6 \mathrm{~Hz}, 1 \mathrm{H}), 1.77-1.63(\mathrm{~m}$, $4 \mathrm{H}), 1.54(\mathrm{~d}, \mathrm{~J}=16 \mathrm{~Hz}, 1 \mathrm{H}), 1.38-0.95(\mathrm{~m}, 6 \mathrm{H}) .{ }^{13} \mathrm{C} \mathrm{NMR}\left(100 \mathrm{MHz}, \mathrm{CDCl}_{3}, 25^{\circ} \mathrm{C}\right): \delta 135.4$, $117.9,74.7,43.0,38.8,29.1,28.1,26.5,26.2,26.1$.<smiles>C=CCC(O)C1CCOCC1</smiles>

$2 \mathrm{u}$

2u, $\mathrm{Rf}=0.5$ (PE:EA = 2:1), column solvent: PE:EtOAc = 10:1. 51\%, colorless oil, ${ }^{1} \mathrm{H}$ NMR (400 $\mathrm{MHz}, \mathrm{CDCl}_{3}$ ) $\delta 5.77$ (dddd, $\mathrm{J}=17.5,9.5,7.8,6.5 \mathrm{~Hz}, 1 \mathrm{H}$ ), $5.12-4.98(\mathrm{~m}, 2 \mathrm{H}), 3.91$ (dt, $\mathrm{J}=$ 10.9, $5.7 \mathrm{~Hz}, 2 \mathrm{H}), 3.33-3.30(\mathrm{~m}, 1 \mathrm{H}), 3.30-3.24(\mathrm{~m}, 2 \mathrm{H}), 2.40(\mathrm{~s}, 1 \mathrm{H}), 2.33-2.18(\mathrm{~m}, 1 \mathrm{H})$, $2.14-1.95(\mathrm{~m}, 1 \mathrm{H}), 1.68(\mathrm{ddd}, \mathrm{J}=13.0,3.3,1.8 \mathrm{~Hz}, 1 \mathrm{H}), 1.60-1.23(\mathrm{~m}, 4 \mathrm{H}) .{ }^{13} \mathrm{C}$ NMR $(101$ $\left.\mathrm{MHz}, \mathrm{CDCl}_{3}\right) \delta 134.75,118.36,73.89,67.92,67.70,40.37,38.55,28.89,28.48$. HRMS+pESI $(\mathrm{m} / \mathrm{z})[\mathrm{M}-\mathrm{OH}]^{+}$calculated for $\mathrm{C}_{9} \mathrm{H}_{15} \mathrm{O}, 139.1117$, found 139.1116 .<smiles>C=CCC(O)C1CCCC1</smiles>

2v

2v, Rf $=0.5$ (PE:EA = 5:1), column solvent: $\mathrm{PE}: \mathrm{EtOAc}=30: 1.38 \%$, colorless oil, ${ }^{1} \mathrm{H}$ NMR $(400$ $\left.\mathrm{MHz}, \mathrm{CDCl}_{3}\right) \delta 5.85$ (dddd, $\left.\mathrm{J}=17.1,10.7,8.1,6.3 \mathrm{~Hz}, 1 \mathrm{H}\right), 5.23-4.99(\mathrm{~m}, 2 \mathrm{H}), 3.44$ (td, $\mathrm{J}=$ 8.0, 3.3 Hz, $1 \mathrm{H}), 2.47-2.25(\mathrm{~m}, 1 \mathrm{H}), 2.20-2.03(\mathrm{~m}, 1 \mathrm{H}), 1.97-1.74(\mathrm{~m}, 2 \mathrm{H}), 1.75-1.45(\mathrm{~m}$, $6 \mathrm{H}), 1.37(\mathrm{td}, \mathrm{J}=8.2,4.3 \mathrm{~Hz}, 1 \mathrm{H}), 1.28-1.12(\mathrm{~m}, 1 \mathrm{H}) .{ }^{13} \mathrm{C} \mathrm{NMR}\left(101 \mathrm{MHz}, \mathrm{CDCl}_{3}\right) \delta 135.19$, $117.90,74.69,45.74,40.78,29.05,28.75,25.64,25.57$. HRMS+pESI (m/z) $[\mathrm{M}-\mathrm{OH}]^{+}$ calculated for $\mathrm{C}_{9} \mathrm{H}_{15}, 123.1168$, found 123.1168 .

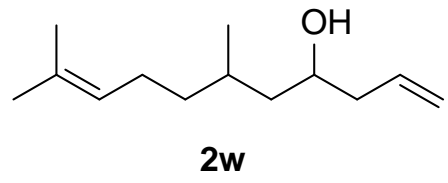

2w, Rf $=0.5$ (PE:EA = 2:1), column solvent: $\mathrm{PE}: \mathrm{EtOAC}=15: 1.68 \%$, colorless oil, ${ }^{1} \mathrm{H}$ NMR $\left(400 \mathrm{MHz}, \mathrm{CDCl}_{3}\right) \delta 5.76(\mathrm{dq}, \mathrm{J}=19.2,8.0,7.6 \mathrm{~Hz}, 1 \mathrm{H}), 5.04(\mathrm{t}, \mathrm{J}=13.0 \mathrm{~Hz}, 3 \mathrm{H}), 3.68$ (dq, $J=$ 8.9, $4.2 \mathrm{~Hz}, 1 \mathrm{H}), 2.37-2.07(\mathrm{~m}, 2 \mathrm{H}), 2.30-1.98(\mathrm{~m}, 2 \mathrm{H}), 1.61(\mathrm{~s}, 3 \mathrm{H}), 1.53(\mathrm{~s}, 3 \mathrm{H}), 1.33(\mathrm{tt}, J$ $=15.2,7.2 \mathrm{~Hz}, 3 \mathrm{H}$ ), 1.09 (dtd, $J=25.4,14.9,12.9,7.3 \mathrm{~Hz}, 2 \mathrm{H}$ ), 0.86 (dd, $J=6.7,2.2 \mathrm{~Hz}, 3 \mathrm{H}$ ). 
${ }^{13} \mathrm{C}$ NMR $\left(101 \mathrm{MHz}, \mathrm{CDCl}_{3}\right) \delta 134.82,131.16,124.73,118.05,68.72,44.28,42.11,36.70$, 29.25, 25.65, 25.31, 20.18, 17.59. Org. Biomol. Chem, 8, 3055; 2010. ${ }^{1} \mathrm{H}$ NMR (400 MHz, $\left.\mathrm{CDCl}_{3}\right) \delta(\mathrm{ppm}): 5.79$ (ddt, J = 16.5, 10.7, $\left.7.4 \mathrm{~Hz}, 1 \mathrm{H}\right), 5.13(\mathrm{bd}, \mathrm{J}=16.5 \mathrm{~Hz}, 1 \mathrm{H}), 5.06(\mathrm{bd}, \mathrm{J}=$ $10.7 \mathrm{~Hz}, 1 \mathrm{H}), 5.06(\mathrm{bt}, \mathrm{J}=6.5 \mathrm{~Hz}, 1 \mathrm{H}), 3.75-3.70(\mathrm{~m}, 1 \mathrm{H}), 2.30-1.94(\mathrm{~m}, 4 \mathrm{H}), 1.66(\mathrm{~s}, 3 \mathrm{H})$, $1.58(\mathrm{~s}, 3 \mathrm{H}), 1.50-1.17(\mathrm{~m}, 5 \mathrm{H}), 0.88(\mathrm{~d}, \mathrm{~J}=6.2 \mathrm{~Hz}, 3 \mathrm{H}, \mathrm{R}, \mathrm{R}-11), 0.84(\mathrm{~d}, \mathrm{~J}=6.2 \mathrm{~Hz}, 3 \mathrm{H}$, $\mathrm{SR}-12) .{ }^{13} \mathrm{C}$ NMR $\left(100 \mathrm{MHz}, \mathrm{CDCl}_{3}\right.$; DEPT) $\delta(\mathrm{ppm}): 134.9(\mathrm{CH}), 131.5(\mathrm{C}), 124.8(\mathrm{CH})$, $118.1\left(\mathrm{CH}_{2}\right), 68.7(\mathrm{CH}), 44.3\left(\mathrm{CH}_{2}\right), 42.2\left(\mathrm{CH}_{2}\right), 37.9\left(\mathrm{CH}_{2}\right), 28.9(\mathrm{CH}), 25.7\left(\mathrm{CH}_{3}\right), 25.4\left(\mathrm{CH}_{2}\right)$, $20.2\left(\mathrm{CH}_{3}\right), 17.7\left(\mathrm{CH}_{3}\right)$.<smiles>C=CCC(O)C1CCN(C(=O)OC(C)(C)C)CC1</smiles>

2x

2x, Rf = 0.6 (PE:EA = 2:1), column solvent: PE:EtOAc $=10: 1.37 \%$, colorless oil, ${ }^{1} \mathrm{H}$ NMR $(400$ $\left.\mathrm{MHz}, \mathrm{CDCl}_{3}\right) \delta 5.91-5.70(\mathrm{~m}, 1 \mathrm{H}), 5.23-5.04(\mathrm{~m}, 2 \mathrm{H}), 4.13(\mathrm{~s}, 2 \mathrm{H}), 3.40(\mathrm{~s}, 1 \mathrm{H}), 2.64(\mathrm{~s}, 2 \mathrm{H})$, $2.34(\mathrm{~d}, \mathrm{~J}=14.0 \mathrm{~Hz}, 1 \mathrm{H}), 2.11(\mathrm{dt}, \mathrm{J}=14.2,8.4 \mathrm{~Hz}, 1 \mathrm{H}), 1.87-1.75(\mathrm{~m}, 1 \mathrm{H}), 1.73(\mathrm{~d}, J=3.7$ $\mathrm{Hz}, 1 \mathrm{H}), 1.58(\mathrm{~s}, 1 \mathrm{H}), 1.44(\mathrm{~s}, 9 \mathrm{H}), 1.23(\mathrm{td}, \mathrm{J}=12.6,4.1 \mathrm{~Hz}, 2 \mathrm{H}) .{ }^{13} \mathrm{C} \mathrm{NMR}\left(101 \mathrm{MHz}, \mathrm{CDCl}_{3}\right)$ $\delta$ 154.79, 134.76, 118.51, 79.27, 73.69, 44.15, 41.45, 38.78, 28.43, 28.08. ACS Catalysis, 9(10), 9158 - 9163; 2019. ${ }^{1} \mathrm{H}$ NMR (500 MHz, $\mathrm{CDCl}_{3}$ ): $\delta 5.76$ (dddd, J = 15.5, 11.5, 8.4, $6.1 \mathrm{~Hz}$, 1H), $5.14-5.05$ (m, 2H), 4.08 (s, 2H), 3.35 (ddd, J = 9.2, 6.1, 3.4 Hz, 1H), $2.59(\mathrm{~s}, 2 \mathrm{H}), 2.29$ (dddd, J = 14.1, 6.3, 3.2, $1.5 \mathrm{~Hz}, 1 \mathrm{H}$ ), 2.06 (dt, J = 14.0, $8.4 \mathrm{~Hz}, 1 \mathrm{H}$ ), 1.75 (dq, J = 11.3, $2.8 \mathrm{~Hz}$, 1H), 1.54 (dt, J = 13.0, 3.0 Hz, 1H), 1.45 (ddq, J = 11.8, 5.7, 3.2 Hz, 1H), 1.39 (s, 9H), $1.26-$ $1.14(\mathrm{~m}, 2 \mathrm{H}) .{ }^{13} \mathrm{C}$ NMR $\left(125 \mathrm{MHz}, \mathrm{CDCl}_{3}\right)$ : $\delta 154.8,134.8,118.6,79.3,73.7,43.8,41.5,38.8$, $28.5,28.1$.

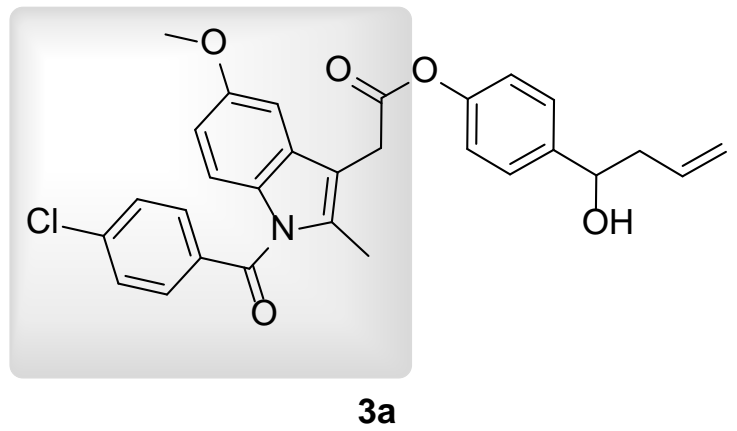

3a, $\mathrm{Rf}=0.4(\mathrm{PE}: \mathrm{EA}=2: 1)$, column solvent: $\mathrm{PE}: \mathrm{EtOAc}=5: 1.44 \%$, white solid, ${ }^{1} \mathrm{H}$ NMR $(400$ $\left.\mathrm{MHz}, \mathrm{CDCl}_{3}\right) \delta 7.67(\mathrm{~d}, \mathrm{~J}=8.3 \mathrm{~Hz}, 2 \mathrm{H}), 7.47(\mathrm{~d}, J=8.3 \mathrm{~Hz}, 2 \mathrm{H}), 7.35(\mathrm{~d}, J=8.2 \mathrm{~Hz}, 2 \mathrm{H}), 7.09$ $-7.02(\mathrm{~m}, 3 \mathrm{H}), 6.90(\mathrm{~d}, \mathrm{~J}=9.0 \mathrm{~Hz}, 1 \mathrm{H}), 6.70(\mathrm{~d}, \mathrm{~J}=9.0 \mathrm{~Hz}, 1 \mathrm{H}), 5.88-5.64(\mathrm{~m}, 1 \mathrm{H}), 5.23-$ $5.06(\mathrm{~m}, 2 \mathrm{H}), 4.81-4.62(\mathrm{~m}, 1 \mathrm{H}), 3.92-3.88(\mathrm{~m}, 2 \mathrm{H}), 3.84(\mathrm{~s}, 3 \mathrm{H}), 2.52-2.48(\mathrm{~m}, 1 \mathrm{H}), 2.45$ (s, 3H), $2.15-2.07(\mathrm{~m}, 1 \mathrm{H}) .{ }^{13} \mathrm{C}$ NMR $\left(101 \mathrm{MHz}, \mathrm{CDCl}_{3}\right) \delta 169.32,168.29,156.07,149.89$, 141.57, 139.32, 136.19, 134.14, 133.77, 131.17, 130.80, 130.46, 129.12, 126.85, 121.27, 118.66, 115.00, 111.96, 111.77, 101.16, 72.63, 55.70, 43.83, 30.52, 13.42. HRMS-ESI (m/z) $[\mathrm{M}+\mathrm{H}]]^{+}$calculated for $\mathrm{C}_{29} \mathrm{H}_{27} \mathrm{ClNO}_{5}, 504.1578$, found 504.1571 . 


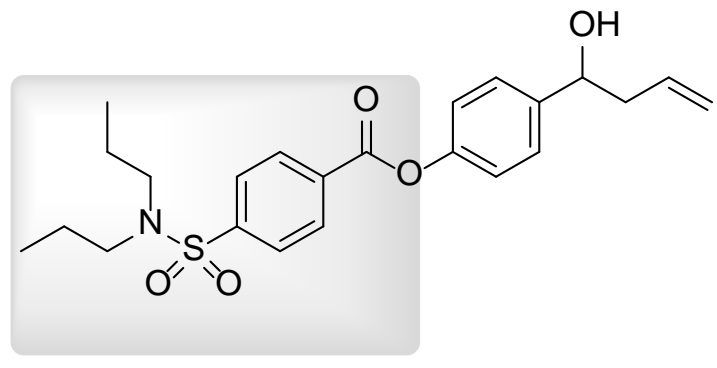

$3 b$

3b, $\mathrm{Rf}=0.4$ (PE:EA = 2:1), column solvent: $\mathrm{PE}: \mathrm{EtOAc}=5: 1.62 \%$, white solid, ${ }^{1} \mathrm{H}$ NMR (400 $\left.\mathrm{MHz}, \mathrm{CDCl}_{3}\right) \delta 8.31(\mathrm{~d}, \mathrm{~J}=8.6 \mathrm{~Hz}, 2 \mathrm{H}), 7.94(\mathrm{~d}, \mathrm{~J}=8.6 \mathrm{~Hz}, 2 \mathrm{H}), 7.44(\mathrm{~d}, \mathrm{~J}=8.5 \mathrm{~Hz}, 2 \mathrm{H}), 7.20$ $(\mathrm{d}, J=8.6 \mathrm{~Hz}, 2 \mathrm{H}), 5.91-5.74(\mathrm{~m}, 1 \mathrm{H}), 5.23-5.10(\mathrm{~m}, 2 \mathrm{H}), 4.78$ (ddd, $\mathrm{J}=8.0,4.9,3.1 \mathrm{~Hz}$, $1 \mathrm{H}), 3.18-3.06(\mathrm{~m}, 4 \mathrm{H}), 2.62-2.42(\mathrm{~m}, 2 \mathrm{H}), 2.18(\mathrm{~d}, \mathrm{~J}=3.2 \mathrm{~Hz}, 1 \mathrm{H}), 1.62-1.50(\mathrm{~m}, 4 \mathrm{H})$, $0.88(\mathrm{t}, \mathrm{J}=7.4 \mathrm{~Hz}, 6 \mathrm{H}) .{ }^{13} \mathrm{C}$ NMR $\left(101 \mathrm{MHz}, \mathrm{CDCl}_{3}\right) \delta 163.87,149.79,144.85,141.92,134.15$, $132.74,130.76,127.12,127.04,121.36,118.71,72.63,49.88,43.88,21.89,11.13$. HRMS$\mathrm{ESI}(\mathrm{m} / \mathrm{z})[\mathrm{M}+\mathrm{H}]^{+}$calculated for $\mathrm{C}_{23} \mathrm{H}_{30} \mathrm{NO}_{5} \mathrm{~S}, 432.1845$, found 432.1845.

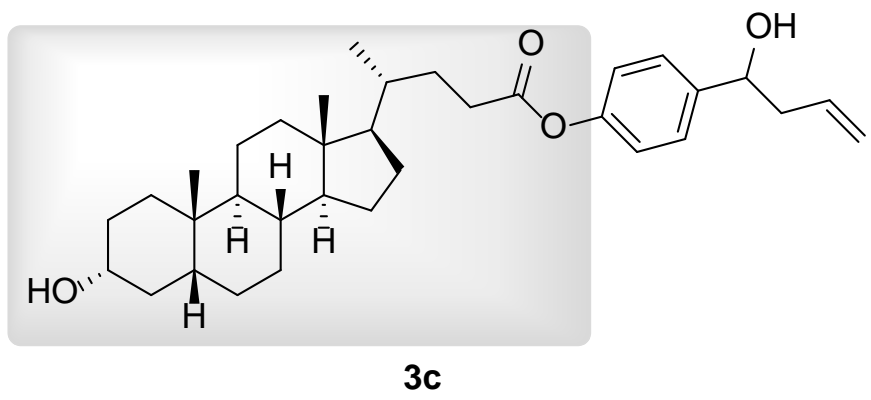

3c, $\mathrm{Rf}=0.4(\mathrm{PE}: \mathrm{EA}=1: 1)$, column solvent: $\mathrm{PE}: \mathrm{EtOAc}=3: 1.69 \%$, white solid, ${ }^{1} \mathrm{H}$ NMR $(400$ $\left.\mathrm{MHz}, \mathrm{CDCl}_{3}\right) \delta 7.35(\mathrm{~d}, \mathrm{~J}=2.7 \mathrm{~Hz}, 2 \mathrm{H}), 7.05(\mathrm{~d}, \mathrm{~J}=8.5 \mathrm{~Hz}, 2 \mathrm{H}), 5.89-5.71(\mathrm{~m}, 1 \mathrm{H}), 5.21-$ $5.07(\mathrm{~m}, 2 \mathrm{H}), 4.78-4.65(\mathrm{~m}, 1 \mathrm{H}), 3.68-3.54(\mathrm{~m}, 1 \mathrm{H}), 2.67-2.40(\mathrm{~m}, 4 \mathrm{H}), 2.02-1.58(\mathrm{~m}$, 10H), $1.56-1.02(\mathrm{~m}, 18 \mathrm{H}), 0.98(\mathrm{~d}, \mathrm{~J}=6.3 \mathrm{~Hz}, 3 \mathrm{H}), 0.92(\mathrm{~s}, 3 \mathrm{H}), 0.66(\mathrm{~s}, 3 \mathrm{H}) .{ }^{13} \mathrm{C}$ NMR $(101$ $\left.\mathrm{MHz}, \mathrm{CDCl}_{3}\right) \delta 172.79,150.01,141.29,134.27,126.83,121.46,118.54,72.74,71.82,56.48$, $55.94,43.81,42.74,42.05,40.40,40.15,36.40,35.82,35.35,35.31,34.54,31.35,30.93$, $30.50,28.22,27.15,26.39,24.18,23.34,22.63,20.80,18.29,14.24,12.04$. HRMS-ESI (m/z) $[\mathrm{M}+\mathrm{Na}]^{+}$calculated for $\mathrm{C}_{34} \mathrm{H}_{50} \mathrm{NaO}_{4}, 545.3607$, found 545.3604 .<smiles>C=CC(c1ccccc1)[C@H](O)c1ccc(OC)cc1</smiles>

$5 a$

5a, $\mathrm{Rf}=0.4$ (PE:EA $=5: 1)$, column solvent: $\mathrm{PE}: \mathrm{EtOAC}=30: 1.89 \%$, colorless oil, ${ }^{1} \mathrm{H}$ NMR $(400$ $\left.\mathrm{MHz}, \mathrm{CDCl}_{3}\right) \delta 7.25-7.17(\mathrm{~m}, 2 \mathrm{H}), 7.15(\mathrm{~d}, \mathrm{~J}=7.4 \mathrm{~Hz}, 1 \mathrm{H}), 7.06(\mathrm{t}, \mathrm{J}=7.1 \mathrm{~Hz}, 4 \mathrm{H}), 6.74(\mathrm{~d}, \mathrm{~J}$ $=8.5 \mathrm{~Hz}, 2 \mathrm{H}), 6.25(\mathrm{dt}, J=17.2,9.6 \mathrm{~Hz}, 1 \mathrm{H}), 5.35-5.18(\mathrm{~m}, 2 \mathrm{H}), 4.80(\mathrm{~d}, J=7.9 \mathrm{~Hz}, 1 \mathrm{H})$, $3.75(\mathrm{~s}, 3 \mathrm{H}), 3.53(\mathrm{t}, \mathrm{J}=8.4 \mathrm{~Hz}, 1 \mathrm{H}), 2.35(\mathrm{~s}, 1 \mathrm{H}) .{ }^{13} \mathrm{C} \mathrm{NMR}\left(101 \mathrm{MHz}, \mathrm{CDCl}_{3}\right) \delta 158.75$, $140.68,138.15,133.95,128.28,127.78,126.46,118.20,113.25,59.23$, 55.10. Organic Letters, 7(12), $2333-2335 ; 2005 .{ }^{1} \mathrm{H}$ NMR $\left(300 \mathrm{MHz}, \mathrm{CDCl}_{3}\right) \delta 7.26-7.04(\mathrm{~m}, 10 \mathrm{H}, \mathrm{Ar}-\mathrm{H})$, 
6.26 (ddd, $\mathrm{J}=16.8,10.2$ and $8.7 \mathrm{~Hz}, 1 \mathrm{H}, \mathrm{CH}), 5.29-5.21\left(\mathrm{~m}, 2 \mathrm{H}, \mathrm{CH}_{2}\right), 4.85$ (dd, $\mathrm{J}=7.8$ and $2.4 \mathrm{~Hz}, 1 \mathrm{H}, \mathrm{CH}), 3.55(\mathrm{t}, \mathrm{J}=8.4 \mathrm{~Hz}, 1 \mathrm{H}, \mathrm{CH}), 2.30(\mathrm{~d}, \mathrm{~J}=2.7 \mathrm{~Hz}, 1 \mathrm{H}, \mathrm{OH}) .{ }^{13} \mathrm{C}$ NMR $(75 \mathrm{MHz}$, $\left.\mathrm{CDCl}_{3}\right) \delta 142.1,140.9,138.1,128.6,128.2,127.7,126.9,118.7,77.5,59.5$.<smiles>C=C[C@H](c1ccccc1)[C@H](O)c1ccccc1-c1ccccc1</smiles>

$5 b$

5b, $\mathrm{Rf}=0.6(\mathrm{PE}: \mathrm{EA}=5: 1)$, column solvent: $\mathrm{PE}:$ EtOAc $=30: 1.73 \%$, yellow oil, ${ }^{1} \mathrm{H}$ NMR $(400$ $\left.\mathrm{MHz}, \mathrm{CDCl}_{3}\right) \delta 7.71(\mathrm{~d}, \mathrm{~J}=7.8 \mathrm{~Hz}, 1 \mathrm{H}), 7.45-7.35(\mathrm{~m}, 4 \mathrm{H}), 7.26(\mathrm{dd}, \mathrm{J}=14.9,1.2 \mathrm{~Hz}, 1 \mathrm{H})$, $7.15-6.99(\mathrm{~m}, 6 \mathrm{H}), 6.72(\mathrm{dd}, J=6.5,3.0 \mathrm{~Hz}, 2 \mathrm{H}), 6.22-6.08(\mathrm{~m}, 1 \mathrm{H}), 5.25-5.09(\mathrm{~m}, 2 \mathrm{H})$, $5.04(\mathrm{dd}, J=7.5,2.3 \mathrm{~Hz}, 1 \mathrm{H}), 3.60-3.51(\mathrm{~m}, 1 \mathrm{H}), 2.23(\mathrm{~d}, \mathrm{~J}=2.6 \mathrm{~Hz}, 1 \mathrm{H}) .{ }^{13} \mathrm{C}$ NMR $(101 \mathrm{MHz}$, $\left.\mathrm{CDCl}_{3}\right) \delta 141.47,140.93,140.64,138.99,137.48,129.63,129.34,128.19,128.03,127.96$, $127.40,127.10,126.86,126.64,126.27,118.35,72.90,57.81 . \mathrm{HRMS}+\mathrm{pESI}(\mathrm{m} / \mathrm{z})[\mathrm{M}-\mathrm{OH}]^{+}$ calculated for $\mathrm{C}_{22} \mathrm{H}_{20} \mathrm{O}, 283.1481$, found 283.1490 .<smiles>C=C[C@@H](c1ccccc1)[C@H](O)c1ccc2ccccc2c1</smiles>

$5 c$

5c, $\mathrm{Rf}=0.4(\mathrm{PE}: \mathrm{EA}=5: 1)$, column solvent: $\mathrm{PE}:$ EtOAc $=30: 1.62 \%$, yellow oil, ${ }^{1} \mathrm{H}$ NMR $(400$ $\left.\mathrm{MHz}, \mathrm{CDCl}_{3}\right) \delta 7.82-7.67(\mathrm{~m}, 3 \mathrm{H}), 7.62(\mathrm{~s}, 1 \mathrm{H}), 7.44(\mathrm{dd}, \mathrm{J}=6.2,3.2 \mathrm{~Hz}, 2 \mathrm{H}), 7.34-7.29(\mathrm{~m}$, 1H), $7.23-7.05(\mathrm{~m}, 5 \mathrm{H}), 6.30$ (ddd, $\mathrm{J}=17.1,10.2,9.0 \mathrm{~Hz}, 1 \mathrm{H}), 5.33-5.20(\mathrm{~m}, 2 \mathrm{H}), 5.03(\mathrm{dd}$, $J=7.6,2.0 \mathrm{~Hz}, 1 \mathrm{H}), 3.68(\mathrm{t}, J=8.3 \mathrm{~Hz}, 1 \mathrm{H}), 2.45(\mathrm{~d}, J=2.5 \mathrm{~Hz}, 1 \mathrm{H}) .{ }^{13} \mathrm{C}$ NMR $(101 \mathrm{MHz}$, $\left.\mathrm{CDCl}_{3}\right) \delta 141.24,140.00,138.41,133.69,133.52,129.08,128.99,128.63,128.24,127.33$, 126.52, 126.35, 125.35, 119.24, 77.93, 59.70. Organic Letters, 22(11), $4418-4423 ; 2020 .{ }^{1} \mathrm{H}$ NMR (400 MHz, CDCl $)$ : $\delta 2.47$ (s, 1H), $3.64(\mathrm{t}, \mathrm{J}=8.0 \mathrm{~Hz}, 1 \mathrm{H}), 4.97(\mathrm{~d}, \mathrm{~J}=7.6 \mathrm{~Hz}, 1 \mathrm{H}), 5.19-$ $5.26(\mathrm{~m}, 2 \mathrm{H}), 6.21-6.30(\mathrm{~m}, 1 \mathrm{H}), 7.04-7.18(\mathrm{~m}, 5 \mathrm{H}), 7.25(\mathrm{dd}, \mathrm{J}=8.8,1.6 \mathrm{~Hz}, 1 \mathrm{H}), 7.38-$ $7.41(\mathrm{~m}, 2 \mathrm{H}), 7.58(\mathrm{~s}, 1 \mathrm{H}), 7.66-7.76(\mathrm{~m}, 3 \mathrm{H}) .{ }^{13} \mathrm{C} \mathrm{NMR}\left(100 \mathrm{MHz}, \mathrm{CDCl}_{3}\right): \delta$ 59.0, 77.2, $118.5,124.6,125.6,125.8,126.6,127.5,127.9,128.3,128.4,132.8,133.0,137.7,139.3$, 140.5 .<smiles>C=C[C@@H](c1ccccc1)[C@H](O)c1ccccc1</smiles>

5d

5d, $\mathrm{Rf}=0.6$ (PE:EA = 5:1), column solvent: PE:EtOAc $=40: 1.54 \%$, yellow oil, ${ }^{1} \mathrm{H}$ NMR $(400$ $\left.\mathrm{MHz}, \mathrm{CDCl}_{3}\right) \delta 7.27-7.12(\mathrm{~m}, 8 \mathrm{H}), 7.11-7.05(\mathrm{~m}, 2 \mathrm{H}), 6.28$ (ddd, $\mathrm{J}=17.1,10.2,8.9 \mathrm{~Hz}, 1 \mathrm{H}$ ), $5.33-5.18(\mathrm{~m}, 2 \mathrm{H}), 4.90-4.81(\mathrm{~m}, 1 \mathrm{H}), 3.57(\mathrm{t}, \mathrm{J}=8.3 \mathrm{~Hz}, 1 \mathrm{H}), 2.39(\mathrm{~d}, J=2.2 \mathrm{~Hz}, 1 \mathrm{H}) .{ }^{13} \mathrm{C}$ NMR $\left(101 \mathrm{MHz}, \mathrm{CDCl}_{3}\right) \delta 141.79,140.58,137.81,128.72,128.28,127.85,127.35,126.61$, 126.53, 118.35, 77.16, 59.14. Organic Letters, 22(11), 4418 - 4423; 2020. ${ }^{1} \mathrm{H}$ NMR (400 MHz, 
$\left.\mathrm{CDCl}_{3}\right)$ : $\delta 2.38(\mathrm{~s}, 1 \mathrm{H}), 3.53(\mathrm{t}, \mathrm{J}=8.0 \mathrm{~Hz}, 1 \mathrm{H}), 4.81(\mathrm{~d}, \mathrm{~J}=7.6 \mathrm{~Hz}, 1 \mathrm{H}), 5.18-5.26(\mathrm{~m}, 2 \mathrm{H})$, $6.19-6.28(\mathrm{~m}, 1 \mathrm{H}), 7.03-7.04(\mathrm{~m}, 2 \mathrm{H}), 7.11-7.21(\mathrm{~m}, 8 \mathrm{H}) .{ }^{13} \mathrm{C} \mathrm{NMR}\left(100 \mathrm{MHz}, \mathrm{CDCl}_{3}\right): \delta$ $59.1,77.1,118.4,126.5,126.6,127.3,127.8,128.3,128.3,137.8,140.6,141.8$.<smiles>C=CC(c1ccccc1)C(O)c1cccs1</smiles>

$5 e$

5e, $\mathrm{Rf}=0.5$ (PE:EA $=5: 1$ ), column solvent: $\mathrm{PE}:$ EtOAc $=30: 1.56 \%$, yellow oil, ${ }^{1} \mathrm{H}$ NMR $(400$ $\left.\mathrm{MHz}, \mathrm{CDCl}_{3}\right) \delta 7.44-7.23(\mathrm{~m}, 5 \mathrm{H}), 7.01-6.92(\mathrm{~m}, 1 \mathrm{H}), 6.82(\mathrm{dd}, \mathrm{J}=5.0,3.5 \mathrm{~Hz}, 1 \mathrm{H}), 6.66(\mathrm{~d}$, $J=3.1 \mathrm{~Hz}, 1 \mathrm{H}$ ), 6.26 (ddd, $J=17.0,10.3,8.8 \mathrm{~Hz}, 1 \mathrm{H}), 5.37-5.25(\mathrm{~m}, 2 \mathrm{H}), 5.14$ (dd, $J=7.9$, $2.8 \mathrm{~Hz}, 1 \mathrm{H}), 3.63(\mathrm{t}, \mathrm{J}=8.3 \mathrm{~Hz}, 1 \mathrm{H}), 2.57(\mathrm{~d}, \mathrm{~J}=2.9 \mathrm{~Hz}, 1 \mathrm{H}) .{ }^{13} \mathrm{C} \mathrm{NMR}\left(101 \mathrm{MHz}, \mathrm{CDCl}_{3}\right) \delta$ 145.58, 140.38, 137.71, 128.61, 128.19, 126.73, 126.21, 124.59, 124.38, 118.71, 73.27, 59.46 HRMS+pESI (m/z) $[\mathrm{M}-\mathrm{OH}]^{+}$calculated for $\mathrm{C}_{14} \mathrm{H}_{13} \mathrm{~S}, 213.0732$, found 213.0735 .<smiles>C=CC(c1ccccc1)C(O)c1ccco1</smiles>

$5 f$

5f, $\mathrm{Rf}=0.5$ (PE:EA $=5: 1)$, column solvent: PE:EtOAc $=30: 1.81 \%$, yellow oil, ${ }^{1} \mathrm{H}$ NMR $(400$ $\left.\mathrm{MHz}, \mathrm{CDCl}_{3}\right) \delta 7.35-7.31(\mathrm{~m}, 1 \mathrm{H}), 7.28-7.24(\mathrm{~m}, 2 \mathrm{H}), 7.21-7.17(\mathrm{~m}, 1 \mathrm{H}), 7.17$ - $7.12(\mathrm{~m}$, $2 \mathrm{H}), 6.29-6.16(\mathrm{~m}, 2 \mathrm{H}), 6.06(\mathrm{~d}, \mathrm{~J}=3.2 \mathrm{~Hz}, 1 \mathrm{H}), 5.32-5.21(\mathrm{~m}, 2 \mathrm{H}), 4.90(\mathrm{dd}, \mathrm{J}=7.9,4.2 \mathrm{~Hz}$ $1 \mathrm{H}), 3.85(\mathrm{t}, \mathrm{J}=8.2 \mathrm{~Hz}, 1 \mathrm{H}), 2.33(\mathrm{~d}, \mathrm{~J}=4.3 \mathrm{~Hz}, 1 \mathrm{H}) .{ }^{13} \mathrm{C} \mathrm{NMR}\left(101 \mathrm{MHz}, \mathrm{CDCl}_{3}\right) \delta 154.11$, $141.76,140.33,137.57,128.37,128.01,126.71,118.43,110.00,107.50,70.89,55.79$. HRMS+pESI (m/z) $[\mathrm{M}-\mathrm{OH}]^{+}$calculated for $\mathrm{C}_{14} \mathrm{H}_{13} \mathrm{O}, 197.0961$, found 197.0961 .<smiles>C=C[C@@H](c1ccccc1)[C@H](O)c1ccc(C)o1</smiles>

$5 \mathrm{~g}$

5g, $\mathrm{Rf}=0.4$ (PE:EA = 5:1), column solvent: $\mathrm{PE}:$ EtOAc $=20: 1.53 \%$, yellow oil, ${ }^{1} \mathrm{H}$ NMR $(400$ $\left.\mathrm{MHz}, \mathrm{CDCl}_{3}\right) \delta 7.32-7.10(\mathrm{~m}, 5 \mathrm{H}), 6.22(\mathrm{dt}, \mathrm{J}=16.9,9.6 \mathrm{~Hz}, 1 \mathrm{H}), 5.91(\mathrm{~d}, \mathrm{~J}=2.8 \mathrm{~Hz}, 1 \mathrm{H})$, $5.77(\mathrm{~s}, 1 \mathrm{H}), 5.34-5.18(\mathrm{~m}, 2 \mathrm{H}), 4.82(\mathrm{dd}, \mathrm{J}=7.9,3.9 \mathrm{~Hz}, 1 \mathrm{H}), 3.83(\mathrm{t}, J=8.3 \mathrm{~Hz}, 1 \mathrm{H}), 2.24$ (s, 4H). ${ }^{13} \mathrm{C}$ NMR $\left(101 \mathrm{MHz}, \mathrm{CDCl}_{3}\right) \delta$ 152.12, 151.53, 140.56, 137.86, 128.36, 128.07, 126.64, $118.37,108.45,105.89,70.85,55.72$, 13.51. HRMS+pESI $(\mathrm{m} / \mathrm{z})[\mathrm{M}-\mathrm{OH}]^{+}$calculated for $\mathrm{C}_{15} \mathrm{H}_{15} \mathrm{O}, 211.1117$, found 211.1120.<smiles>C=C[C@@H](c1ccccc1)[C@H](O)C1CCCCC1</smiles>

$5 \mathrm{~h}$ 
5h, $\mathrm{Rf}=0.6$ (PE:EA = 5:1), column solvent: PE:EtOAc $=30: 1.31 \%$, colorless oil, ${ }^{1} \mathrm{H}$ NMR $(400$ $\left.\mathrm{MHz}, \mathrm{CDCl}_{3}\right) \delta 7.38-7.16(\mathrm{~m}, 5 \mathrm{H}), 6.22-6.07(\mathrm{~m}, 1 \mathrm{H}), 5.27-5.13(\mathrm{~m}, 2 \mathrm{H}), 3.62-3.52(\mathrm{~m}$, $1 \mathrm{H}), 3.51-3.42(\mathrm{~m}, 1 \mathrm{H}), 1.88-1.78(\mathrm{~m}, 1 \mathrm{H}), 1.76-1.69(\mathrm{~m}, 2 \mathrm{H}), 1.68(\mathrm{~s}, 1 \mathrm{H}), 1.60(\mathrm{~s}, 2 \mathrm{H})$, $1.24(\mathrm{~s}, 2 \mathrm{H}), 1.19-1.03(\mathrm{~m}, 4 \mathrm{H}) .{ }^{13} \mathrm{C}$ NMR $\left(101 \mathrm{MHz}, \mathrm{CDCl}_{3}\right) \delta 142.08,138.42,128.69$, $127.93,126.51,117.66,78.08,53.67,39.55,30.18,26.53,26.43,26.33,25.98$. HRMS+pESI $(\mathrm{m} / \mathrm{z})[\mathrm{M}-\mathrm{OH}]^{+}$calculated for $\mathrm{C}_{16} \mathrm{H}_{21}, 213.1638$, found 213.1637 .<smiles>C=C[C@@H](c1ccco1)[C@@H](O)c1ccc(OC)cc1</smiles>

$5 i$

5i, Rf = 0.4 (PE:EA = 5:1), column solvent: PE:EtOAc $=20: 1.76 \%$, colorless oil, ${ }^{1} \mathrm{H}$ NMR $(400$ $\left.\mathrm{MHz}, \mathrm{CDCl}_{3}\right) \delta 7.31(\mathrm{~s}, 1 \mathrm{H}), 7.14(\mathrm{~d}, \mathrm{~J}=8.6 \mathrm{~Hz}, 2 \mathrm{H}), 6.81(\mathrm{~d}, \mathrm{~J}=8.6 \mathrm{~Hz}, 2 \mathrm{H}), 6.26-6.20(\mathrm{~m}$, $1 \mathrm{H}), 6.08(\mathrm{~m}, 1 \mathrm{H}), 5.93(\mathrm{~d}, \mathrm{~J}=3.1 \mathrm{~Hz}, 1 \mathrm{H}), 5.31-5.10(\mathrm{~m}, 2 \mathrm{H}), 4.94(\mathrm{dd}, \mathrm{J}=6.7,2.2 \mathrm{~Hz}, 1 \mathrm{H})$, $3.78(\mathrm{~s}, 3 \mathrm{H}), 3.72-3.61(\mathrm{~m}, 1 \mathrm{H}), 2.29(\mathrm{~d}, \mathrm{~J}=2.6 \mathrm{~Hz}, 1 \mathrm{H}) .{ }^{13} \mathrm{C} \mathrm{NMR}\left(101 \mathrm{MHz}, \mathrm{CDCl}_{3}\right) \delta$ 158.94, 153.99, 141.34, 134.89, 133.83, 127.45, 118.94, 113.40, 110.18, 106.80, 74.99, 55.18, 52.46. HRMS+pESI (m/z) $[\mathrm{M}-\mathrm{OH}]^{+}$calculated for $\mathrm{C}_{15} \mathrm{H}_{15} \mathrm{O}_{2}, 227.1067$, found 227.1067.<smiles>C=CC(c1cccs1)[C@H](O)c1ccc(OC)cc1</smiles>

$5 j$

5j, Rf = 0.4 (PE:EA = 5:1), column solvent: PE:EtOAc $=20: 1.66 \%$, colorless oil, ${ }^{11} \mathrm{H}$ NMR $(400$ $\left.\mathrm{MHz}, \mathrm{CDCl}_{3}\right) \delta 7.19(\mathrm{dd}, \mathrm{J}=21.1,8.6 \mathrm{~Hz}, 5 \mathrm{H}), 6.91(\mathrm{~d}, \mathrm{~J}=3.1 \mathrm{~Hz}, 1 \mathrm{H}), 6.69(\mathrm{~d}, \mathrm{~J}=3.0 \mathrm{~Hz}, 1 \mathrm{H})$, $6.14(\mathrm{dt}, J=18.7,9.5 \mathrm{~Hz}, 1 \mathrm{H}), 5.30-5.16(\mathrm{~m}, 2 \mathrm{H}), 4.85-4.80(\mathrm{~m}, 1 \mathrm{H}), 3.93(\mathrm{t}, J=7.7 \mathrm{~Hz}, 1 \mathrm{H})$, $3.80(\mathrm{~s}, 3 \mathrm{H}), 2.29(\mathrm{~d}, \mathrm{~J}=2.4 \mathrm{~Hz}, 1 \mathrm{H}) .{ }^{13} \mathrm{C}$ NMR $\left(101 \mathrm{MHz}, \mathrm{CDCl}_{3}\right) \delta 159.17,143.49,137.21$, 133.70, 128.03, 126.76, 125.62, 124.49, 118.54, 113.52, 77.00, 55.19, 53.90. HRMS+pESI $(\mathrm{m} / \mathrm{z})[\mathrm{M}-\mathrm{OH}]^{+}$calculated for $\mathrm{C}_{15} \mathrm{H}_{15} \mathrm{OS}, 243.0838$, found 243.0838 .<smiles>C=CC(c1cccs1)[C@H](O)c1ccc(C)cc1</smiles>

$5 k$

5k, Rf $=0.5$ (PE:EA = 5:1), column solvent: PE:EtOAc $=30: 1.62 \%$, colorless oil, ${ }^{1} \mathrm{H}$ NMR $(400$ $\left.\mathrm{MHz}, \mathrm{CDCl}_{3}\right) \delta 7.12(\mathrm{~d}, \mathrm{~J}=13.0 \mathrm{~Hz}, 5 \mathrm{H}), 6.92-6.89(\mathrm{~m}, 1 \mathrm{H}), 6.72(\mathrm{~d}, \mathrm{~J}=3.1 \mathrm{~Hz}, 1 \mathrm{H}), 6.14(\mathrm{dt}$, $J=17.7,9.4 \mathrm{~Hz}, 1 \mathrm{H}), 5.23(\mathrm{dd}, \mathrm{J}=24.9,13.6 \mathrm{~Hz}, 2 \mathrm{H}), 4.85(\mathrm{~d}, \mathrm{~J}=2.3 \mathrm{~Hz}, 1 \mathrm{H}), 3.94-3.84(\mathrm{~m}$, $1 \mathrm{H}), 2.32(\mathrm{~s}, 3 \mathrm{H}), 2.29(\mathrm{~d}, \mathrm{~J}=2.3 \mathrm{~Hz}, 1 \mathrm{H}) .{ }^{13} \mathrm{C} \mathrm{NMR}\left(101 \mathrm{MHz}, \mathrm{CDCl}_{3}\right) \delta$ 143.56, 138.56, 137.46, 137.04, 128.73, 126.78, 126.50, 124.87, 123.87, 118.55, 77.21, 53.71, 21.12. HRMS+pESI (m/z) $[\mathrm{M}-\mathrm{OH}]^{+}$calculated for $\mathrm{C}_{15} \mathrm{H}_{15} \mathrm{~S}, 227.0889$, found 227.0888 .<smiles>C=CC(c1ccccc1)[C@H](O)c1ccccc1</smiles>

$5 \mathbf{l}$ 
5I, Rf $=0.5$ (PE:EA = 5:1), column solvent: PE:EtOAc $=30: 1.50 \%$, colorless oil, ${ }^{1} \mathrm{H}$ NMR $(400$ $\left.\mathrm{MHz}, \mathrm{CDCl}_{3}\right) \delta 7.25(\mathrm{t}, \mathrm{J}=8.2 \mathrm{~Hz}, 5 \mathrm{H}), 7.15-7.11(\mathrm{~m}, 1 \mathrm{H}), 6.89(\mathrm{~d}, \mathrm{~J}=4.1 \mathrm{~Hz}, 1 \mathrm{H}), 6.70(\mathrm{~d}, \mathrm{~J}$ $=3.4 \mathrm{~Hz}, 1 \mathrm{H}), 6.13(\mathrm{ddd}, J=17.1,10.1,8.8 \mathrm{~Hz}, 1 \mathrm{H}), 5.31-5.14(\mathrm{~m}, 2 \mathrm{H}), 4.87(\mathrm{dt}, J=7.0,3.4$ $\mathrm{Hz}, 1 \mathrm{H}), 3.93-3.84(\mathrm{~m}, 1 \mathrm{H}), 2.34(\mathrm{~d}, \mathrm{~J}=2.9 \mathrm{~Hz}, 1 \mathrm{H}) .{ }^{13} \mathrm{C} \mathrm{NMR}\left(101 \mathrm{MHz}, \mathrm{CDCl}_{3}\right) \delta 143.40$, 141.52 , 136.85, 128.02, 127.61, 126.84, 126.57, 124.89, 123.93, 118.69, 77.38, 53.82. HRMS+pESI (m/z) $[\mathrm{M}-\mathrm{OH}]^{+}$calculated for $\mathrm{C}_{14} \mathrm{H}_{13} \mathrm{~S}, 213.0732$, found 213.0733 .<smiles>C=CC(C)(C)C(O)c1ccc(OC)cc1</smiles>

5m, Rf $=0.5$ (PE:EA $=3: 1$ ), column solvent: PE:EtOAc $=30: 1.24 \%$, colorless oil, ${ }^{1} \mathrm{H}$ NMR $\left(400 \mathrm{MHz}, \mathrm{CDCl}_{3}\right) \delta 7.17(\mathrm{~d}, J=8.6 \mathrm{~Hz}, 2 \mathrm{H}), 6.82(\mathrm{~d}, \mathrm{~J}=8.7 \mathrm{~Hz}, 2 \mathrm{H}), 5.90$ (dd, $J=17.5,10.8$ $\mathrm{Hz}, 1 \mathrm{H}), 5.16-4.91(\mathrm{~m}, 2 \mathrm{H}), 4.31(\mathrm{~s}, 1 \mathrm{H}), 3.75(\mathrm{~s}, 3 \mathrm{H}), 2.47(\mathrm{~s}, 1 \mathrm{H}), 0.99(\mathrm{~s}, 3 \mathrm{H}), 0.94(\mathrm{~s}, 3 \mathrm{H})$. ${ }^{13} \mathrm{C}$ NMR $\left(101 \mathrm{MHz}, \mathrm{CDCl}_{3}\right) \delta 158.91,145.22,132.94,128.82,113.74,112.89,80.28,55.22$, 42.35, 24.52, 20.94. Journal of the American Chemical Society, 129(49), 15134 - 15135; 2007. ${ }^{1} \mathrm{H}$ NMR $\left(400 \mathrm{MHz}, \mathrm{CDCl}_{3}\right): 7.22(\mathrm{~d}, \mathrm{~J}=8.7 \mathrm{~Hz}, 2 \mathrm{H}), 6.85(\mathrm{~d}, \mathrm{~J}=8.7 \mathrm{~Hz}, 2 \mathrm{H}), 5.86$ (dd, J = 17.6, $10.8 \mathrm{~Hz}, 1 \mathrm{H}), 5.13(\mathrm{dd}, \mathrm{J}=10.8,1.2 \mathrm{~Hz}, 1 \mathrm{H}), 5.07(\mathrm{dd}, \mathrm{J}=17.6,1.2,1 \mathrm{H}), 4.39(\mathrm{~d}, \mathrm{~J}=2.3,1 \mathrm{H})$, $3.80(\mathrm{~s}, 3 \mathrm{H}), 1.97(\mathrm{~d}, \mathrm{~J}=2.3 \mathrm{~Hz}, 1 \mathrm{H}), 1.00(\mathrm{~s}, 3 \mathrm{H}), 0.94(\mathrm{~s}, 3 \mathrm{H}) .{ }^{13} \mathrm{C} \mathrm{NMR}\left(100 \mathrm{MHz}, \mathrm{CDCl}_{3}\right)$ : 159.1, 145.4, 133.2, 129.0, 114.0, 113.1, 80.5, 55.5, 42.6, 24.8, 21.2. 


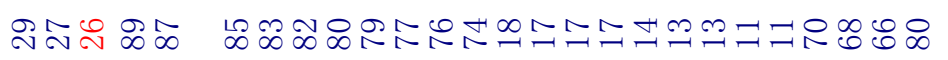

NंN
드웅요 낭

نंi i

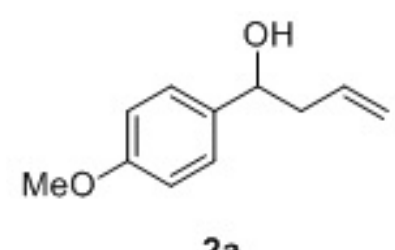

2a

$400 \mathrm{MHz} C D C l_{3}$

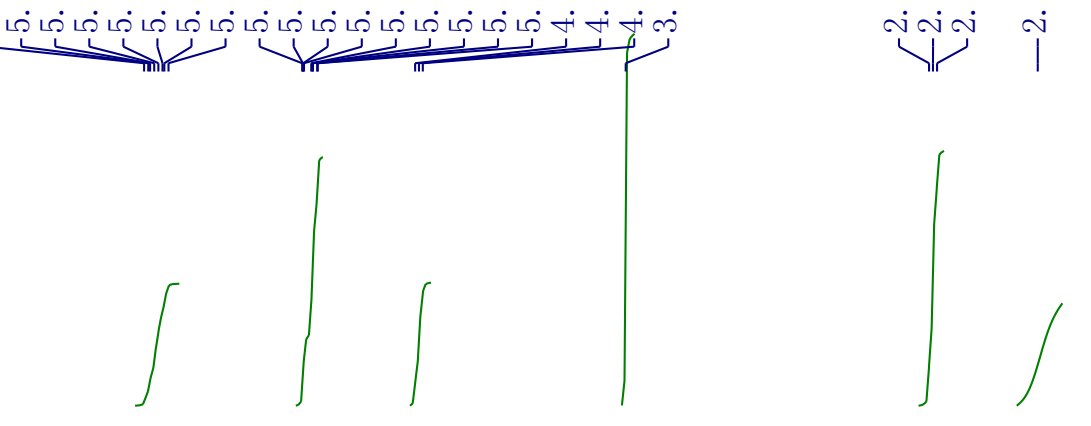

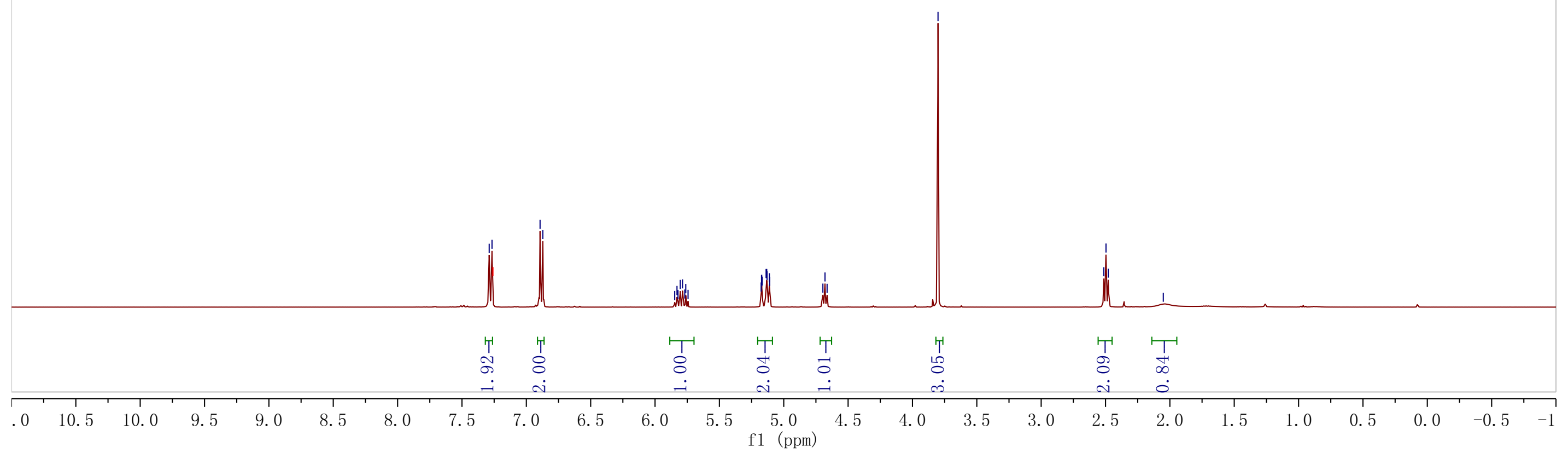




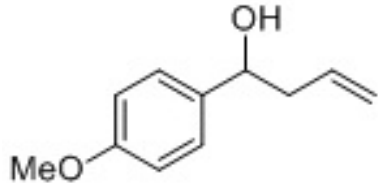

2a

$101 \mathrm{MHz} C D C l_{3}$

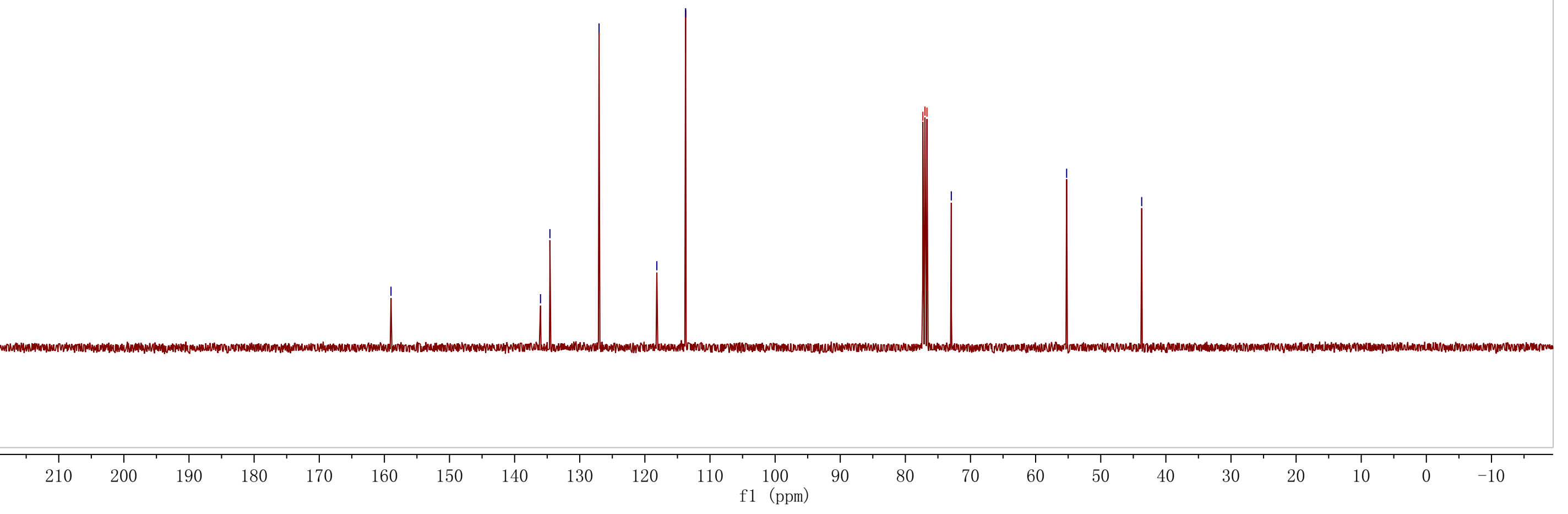




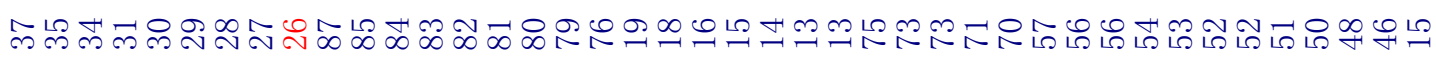

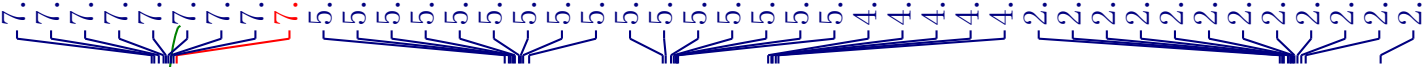

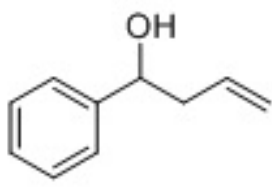

2b

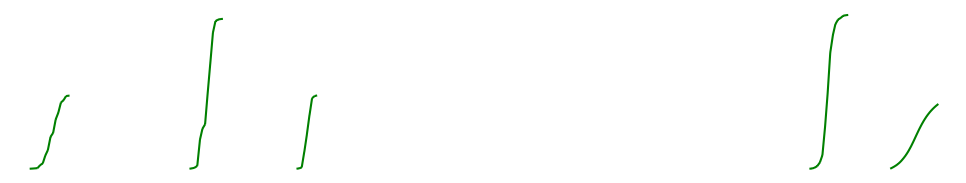

$400 \mathrm{MHz} \mathrm{CDCl}_{3}$

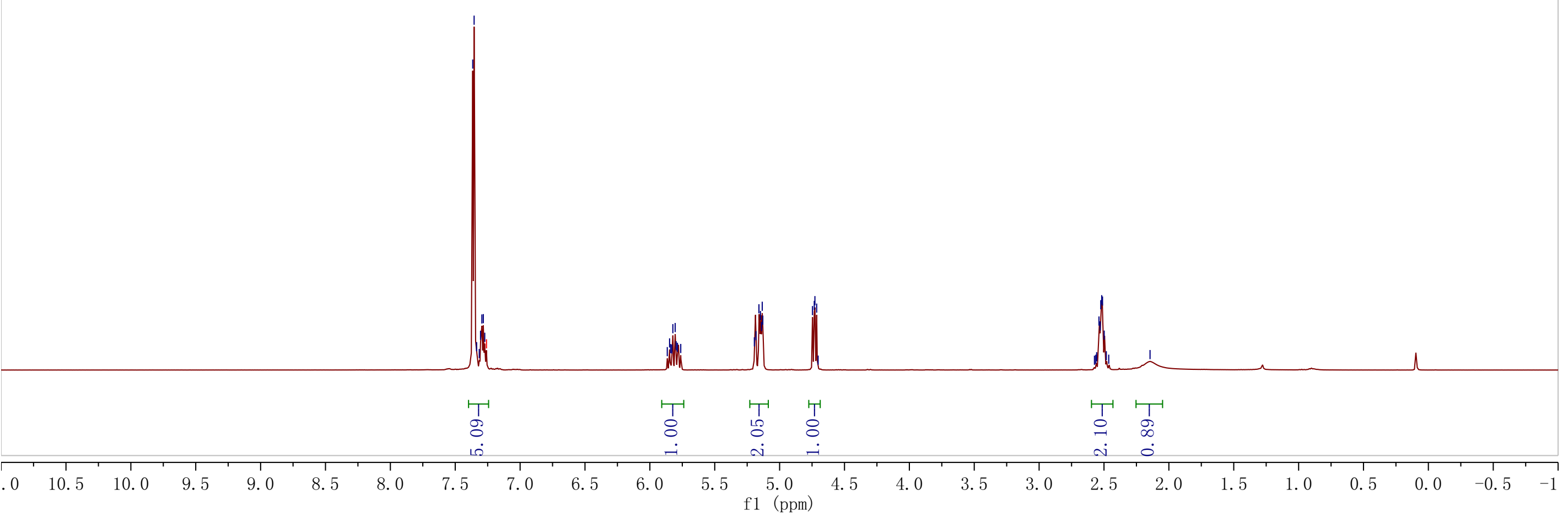




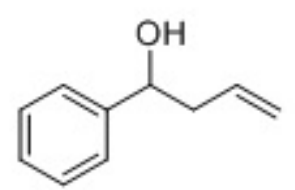

2b

$101 \mathrm{MHz} \mathrm{CDCl}_{3}$

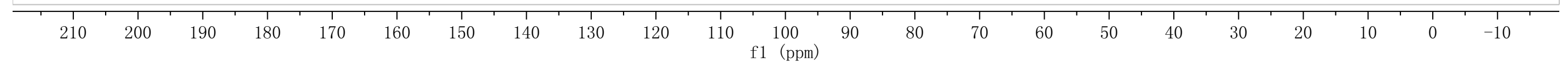




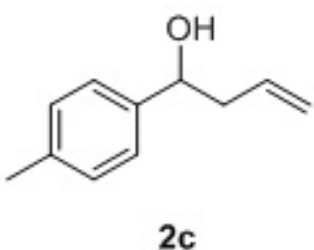

$400 \mathrm{MHz} \mathrm{CDCl}_{3}$
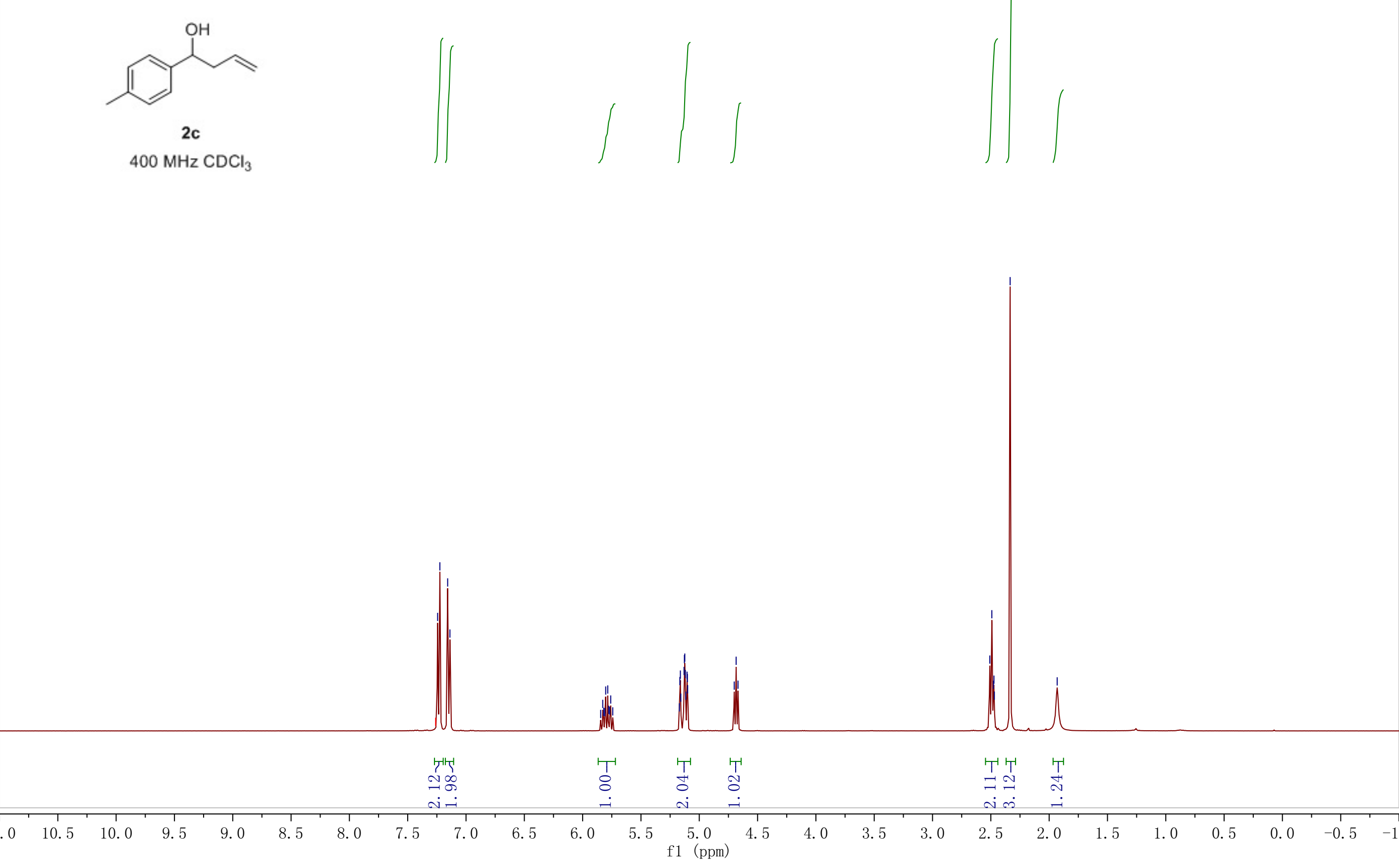


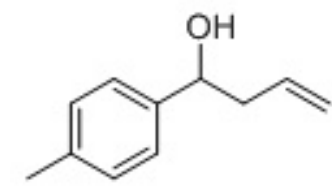

2c

$101 \mathrm{MHz} \mathrm{CDCl}_{3}$

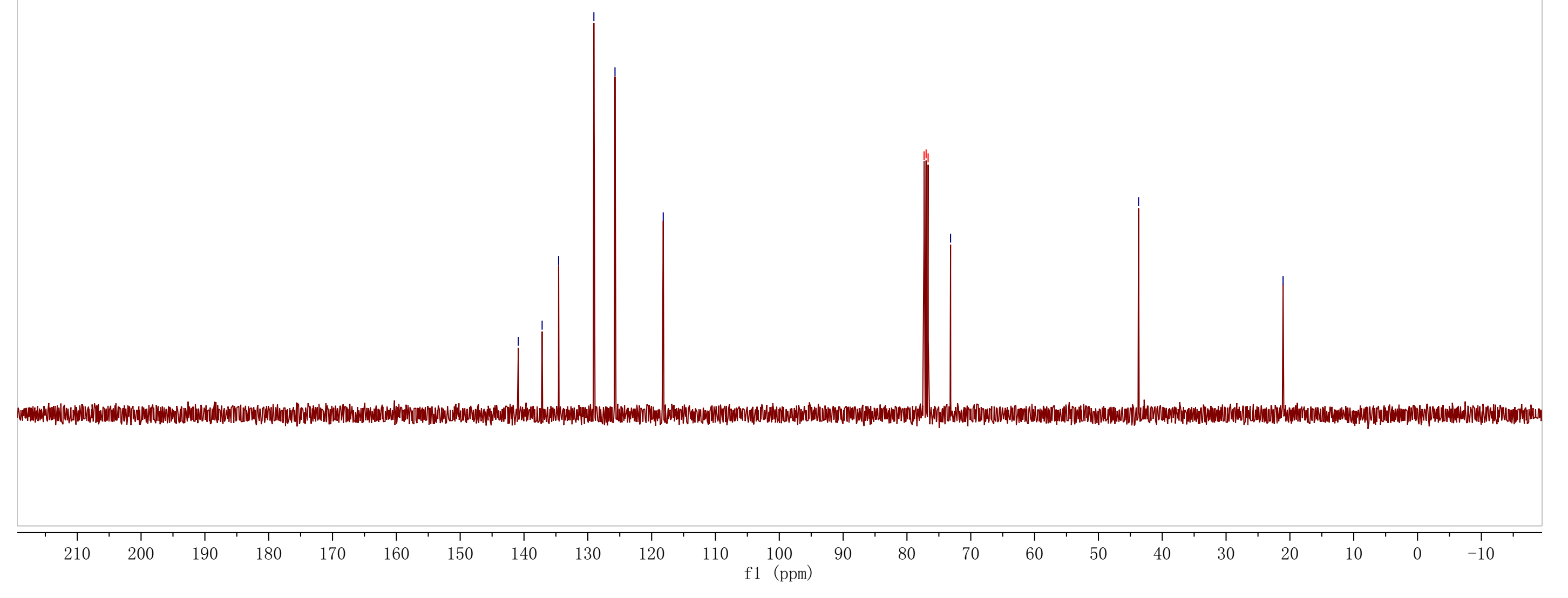



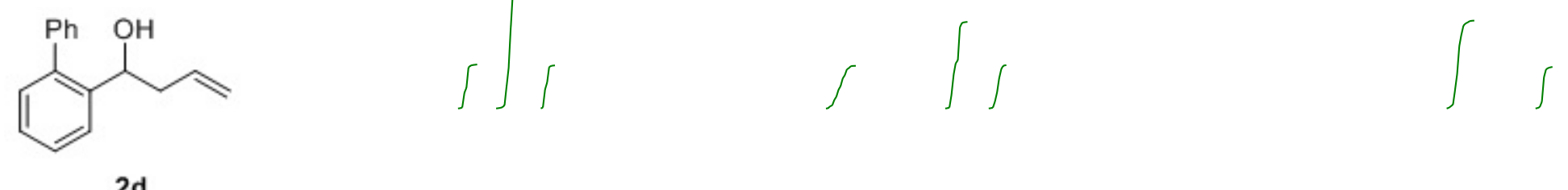

$400 \mathrm{MHz} \mathrm{CDCl}_{3}$

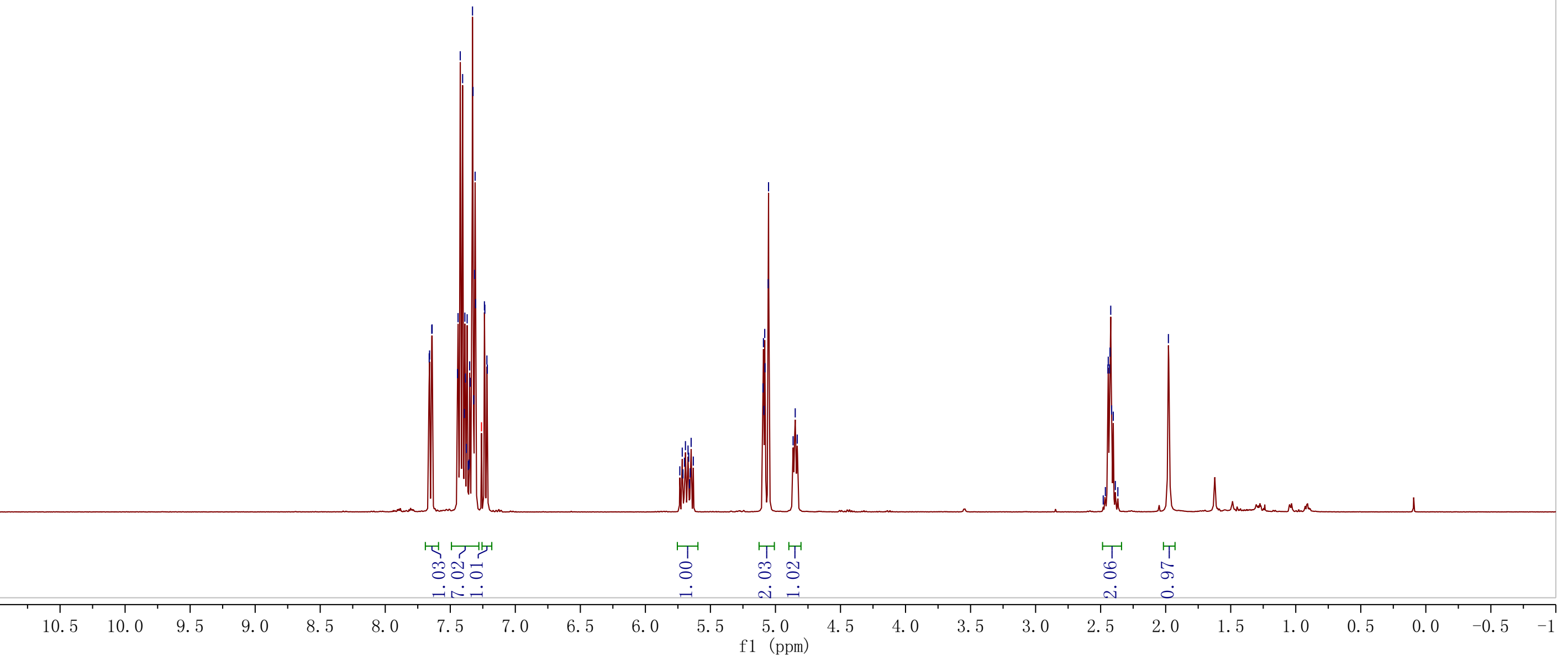



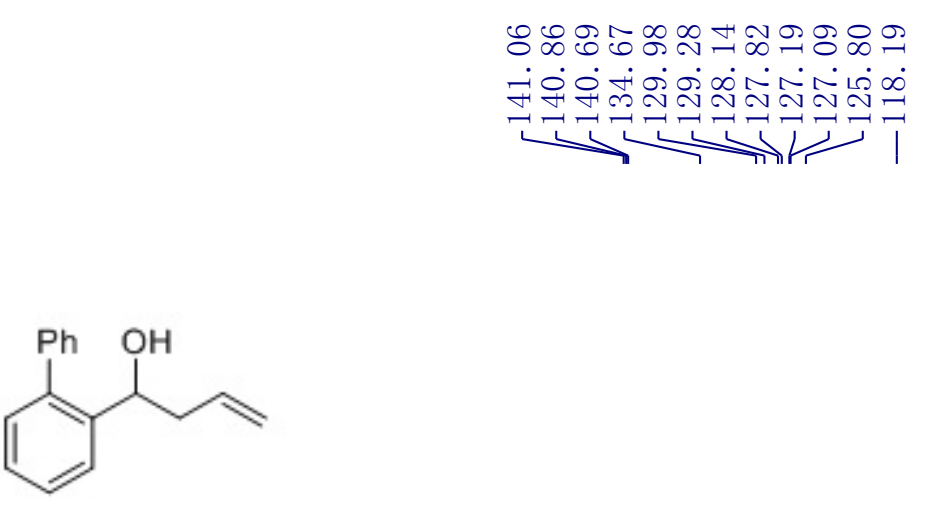

2d

$101 \mathrm{MHz}^{\mathrm{CDCl}} \mathrm{CD}_{3}$

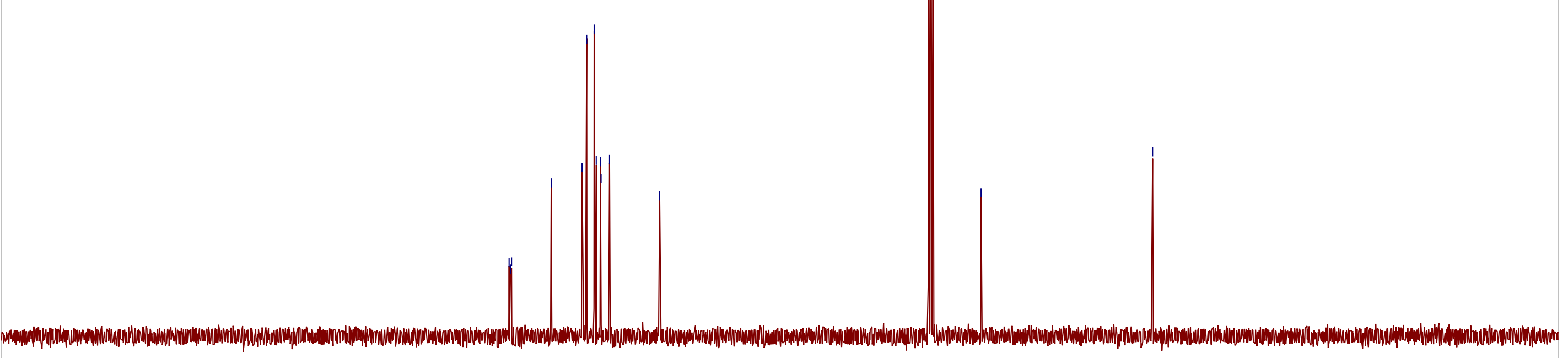

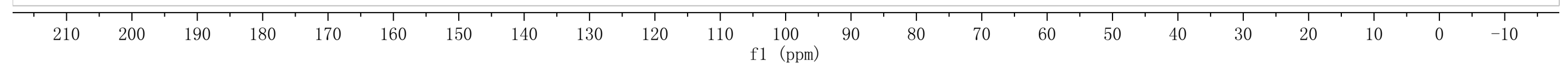




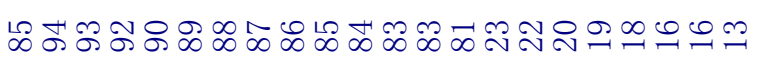

|

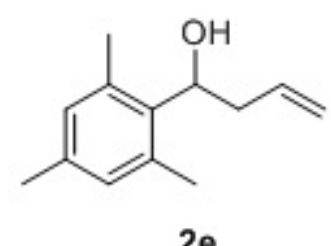

$400 \mathrm{MHz} \mathrm{CDCl}_{3}$

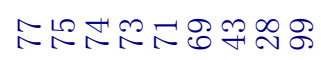

نंivivivini

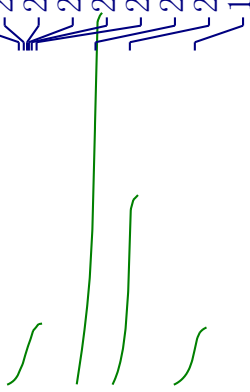

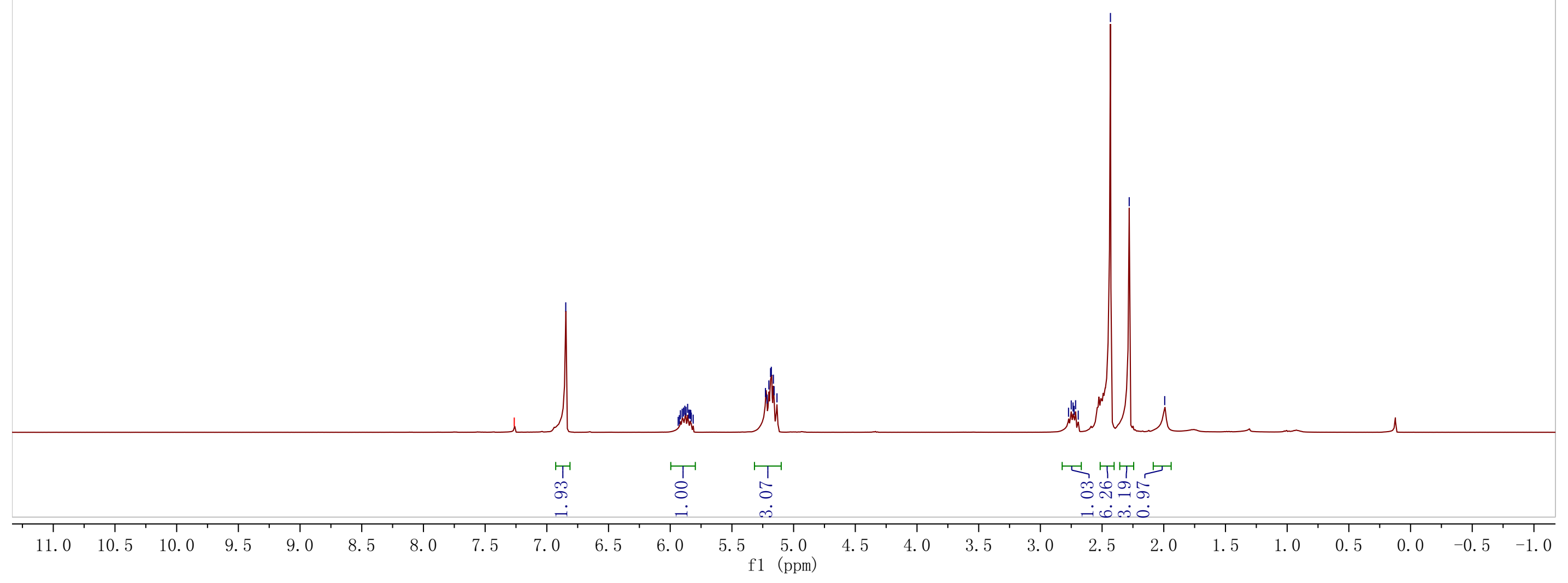




\section{아어에성

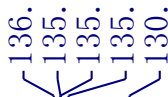 \\ 궁}

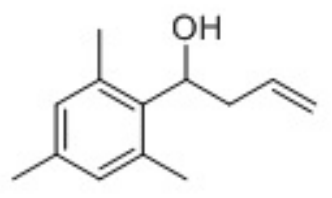

$2 e$

$101 \mathrm{MHz} \mathrm{CDCl}_{3}$

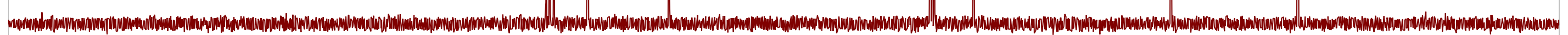

\begin{tabular}{|c|c|c|c|c|c|c|c|c|c|c|c|c|c|c|c|c|c|c|c|c|c|c|}
\hline 210 & 200 & 190 & 180 & 170 & 160 & 150 & 140 & 130 & 120 & 110 & $\begin{array}{c}100 \\
\mathrm{f} 1(\mathrm{ppm})\end{array}$ & 90 & 80 & 70 & 60 & 50 & 40 & 30 & 20 & 10 & 0 & -10 \\
\hline
\end{tabular}




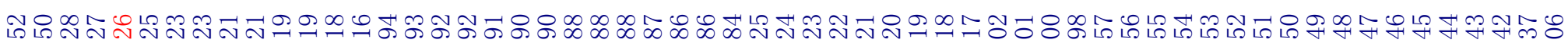

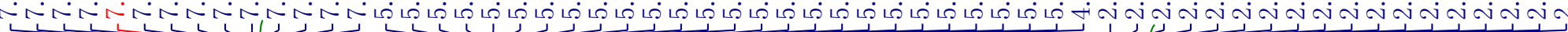
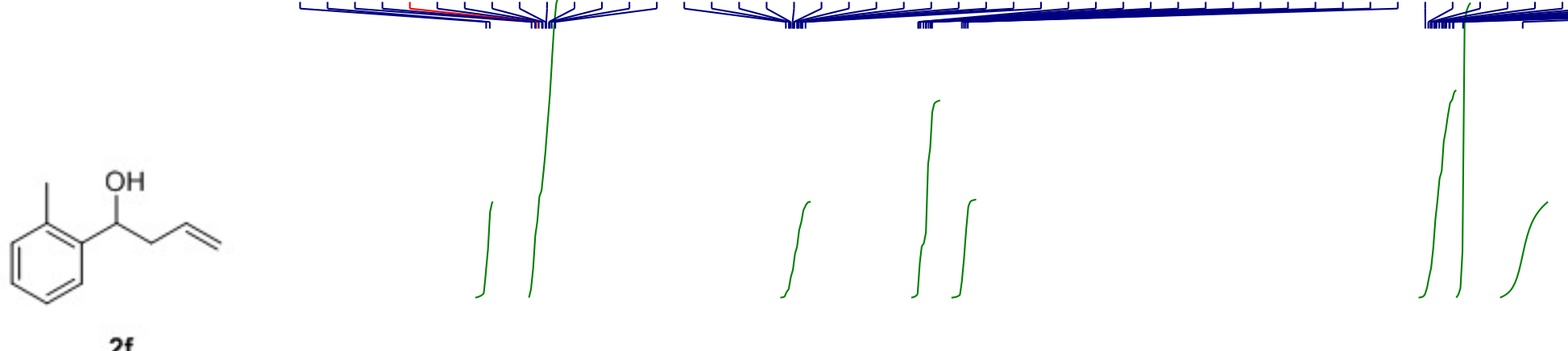

$400 \mathrm{MHz} \mathrm{CDCl}_{3}$

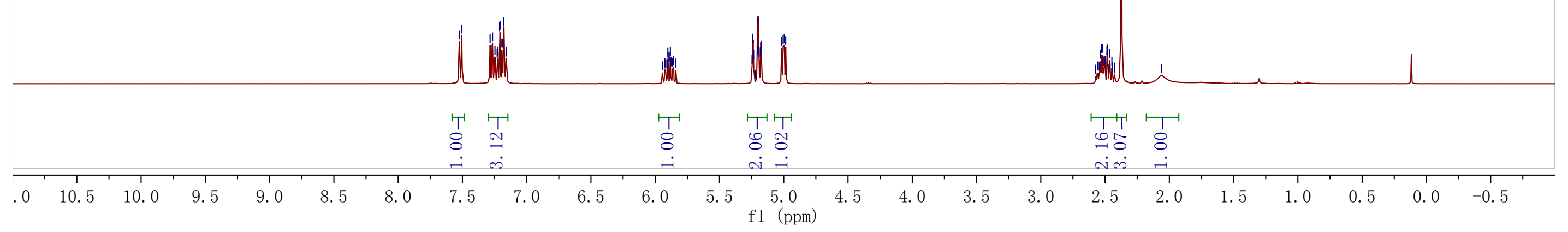




\section{क유 \\ |}

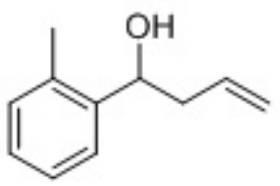

$2 f$

$101 \mathrm{MHz} \mathrm{CDCl}_{3}$

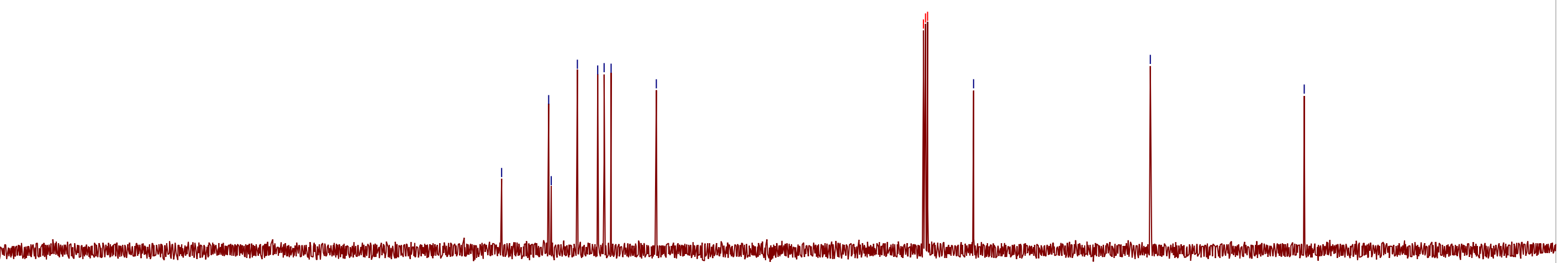

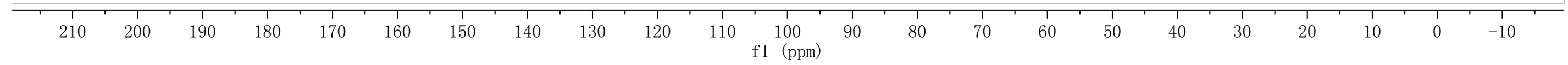




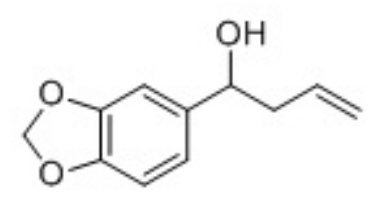

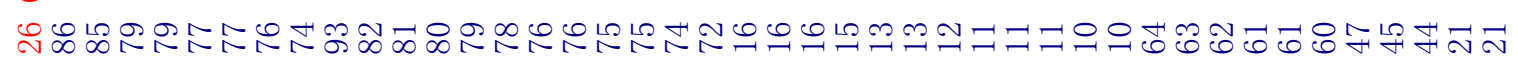

$\sim$ ن

2g

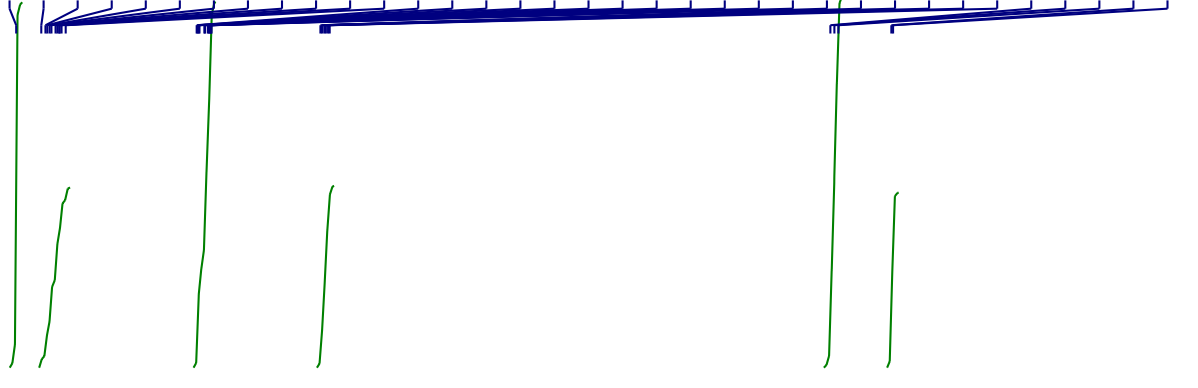

$400 \mathrm{MHz} \mathrm{CDCl}_{3}$

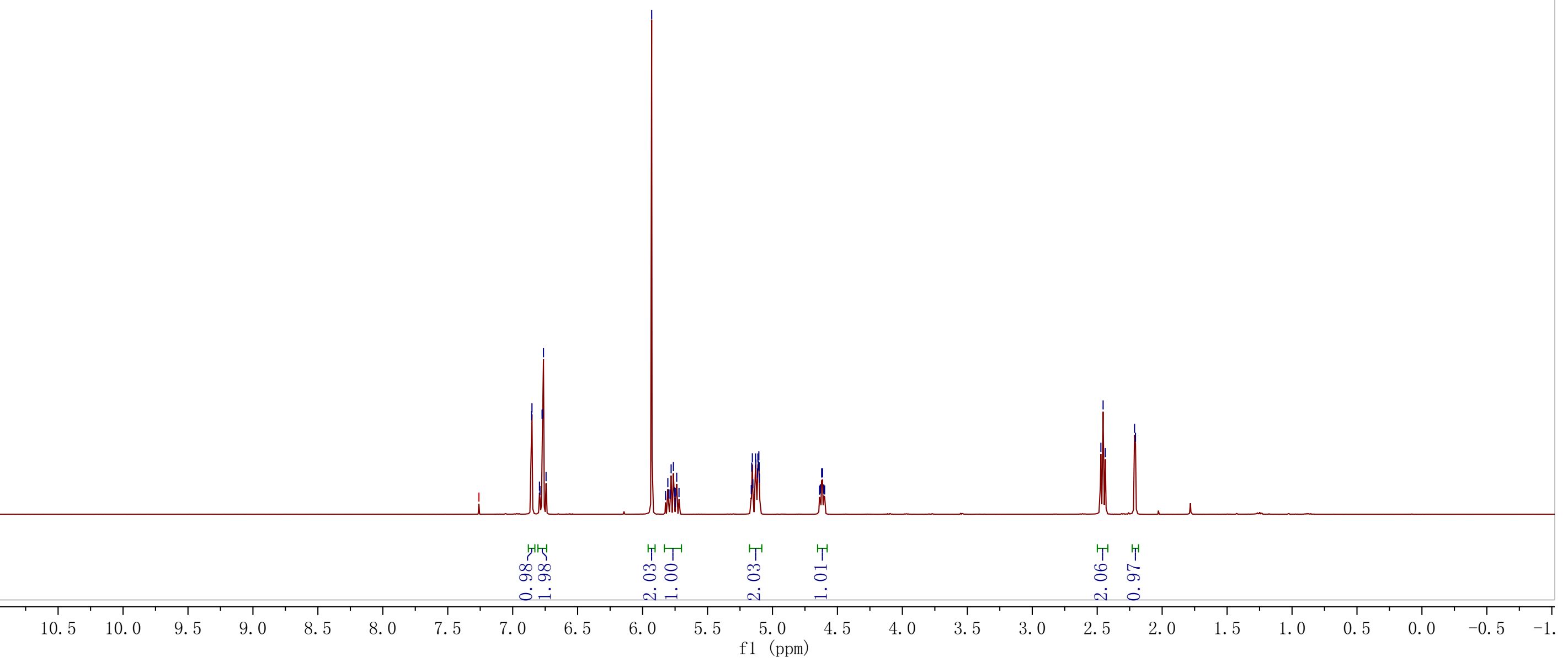




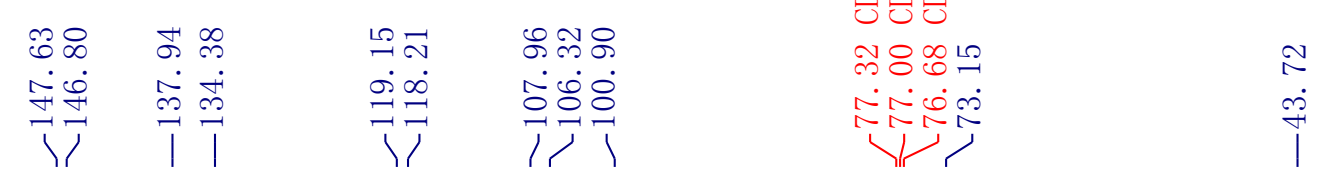

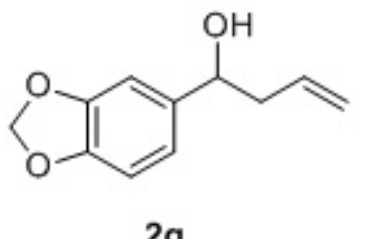

$101 \mathrm{MHz} \mathrm{CDCl}_{3}$

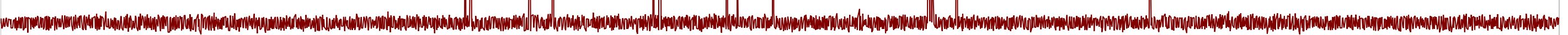




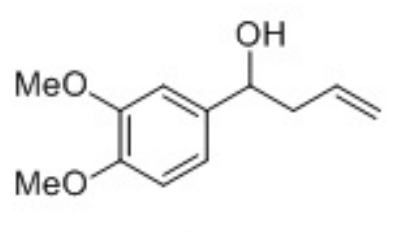

\section{을}

๙ สু

ب ن

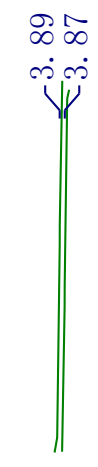

논요

ขึ่

2h

$400 \mathrm{MHz} \mathrm{CDCl}_{3}$

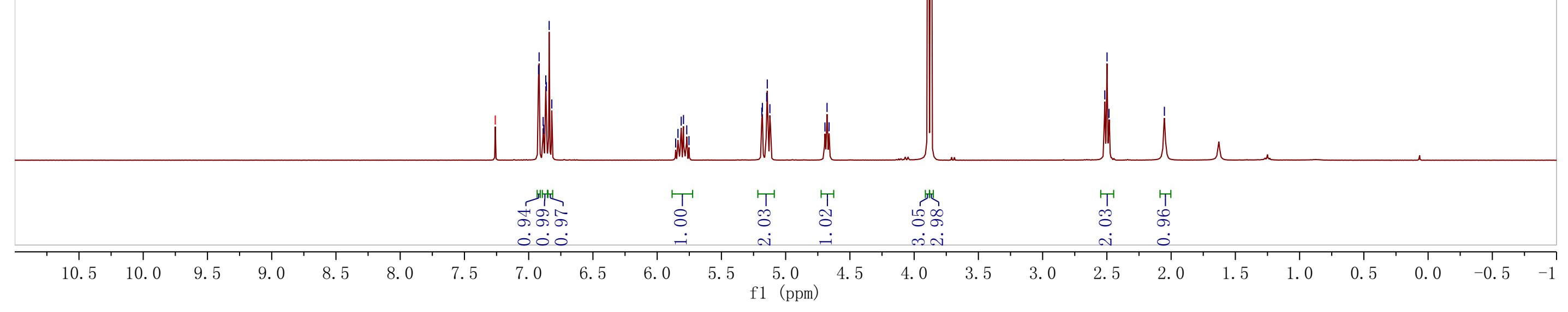




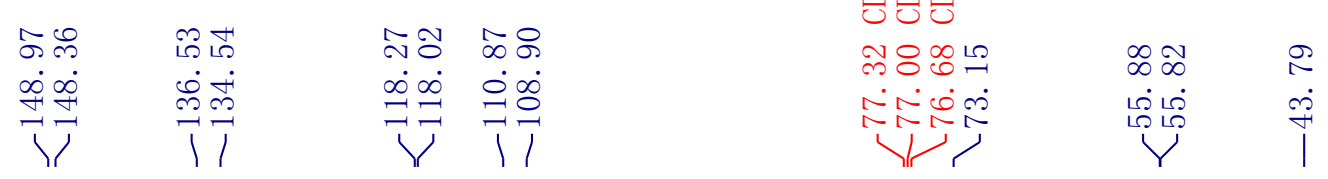

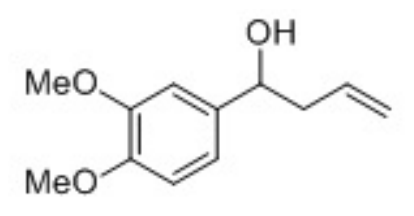

2h

$101 \mathrm{MHz} C D C l_{3}$

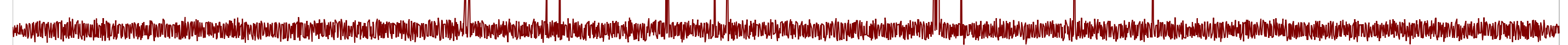

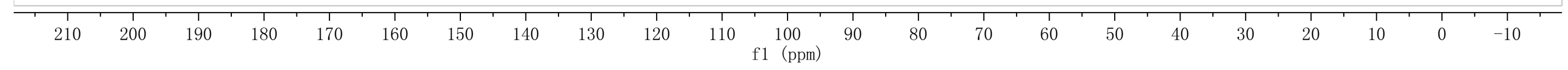



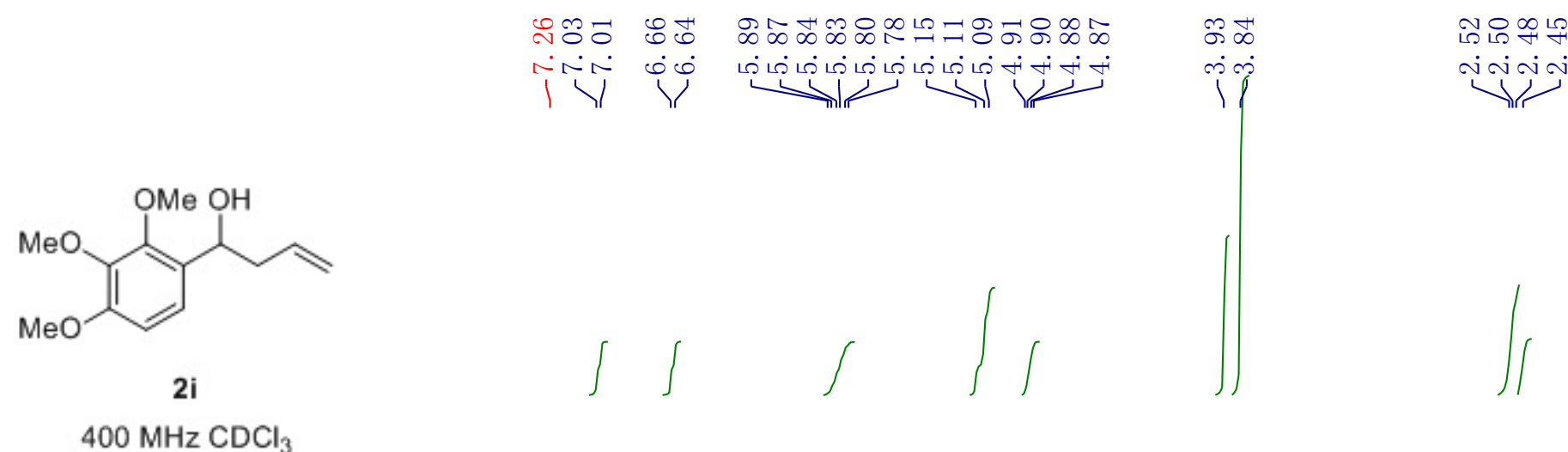

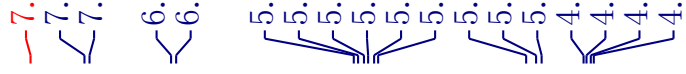

$400 \mathrm{MHz} \mathrm{CDCl}_{3}$
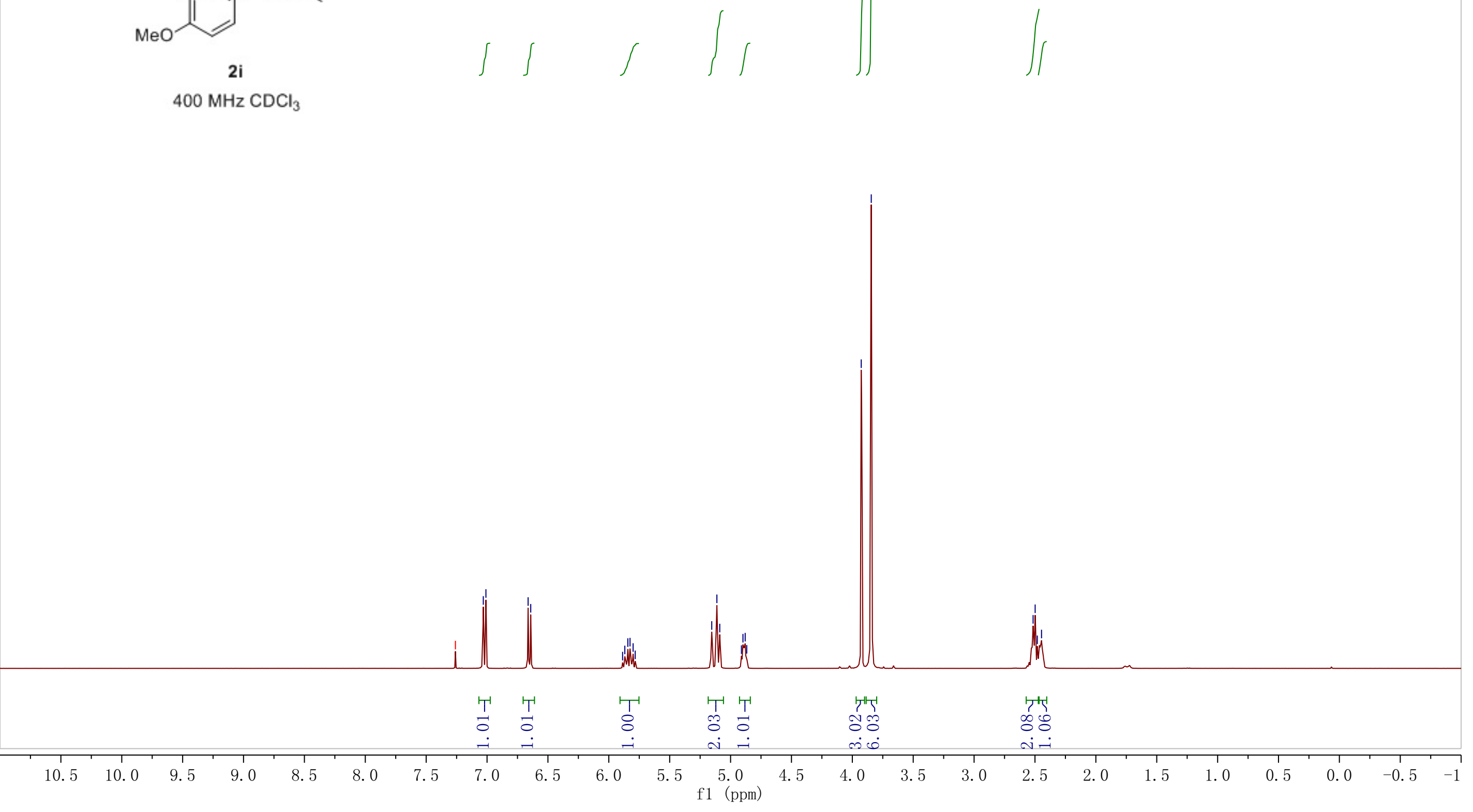


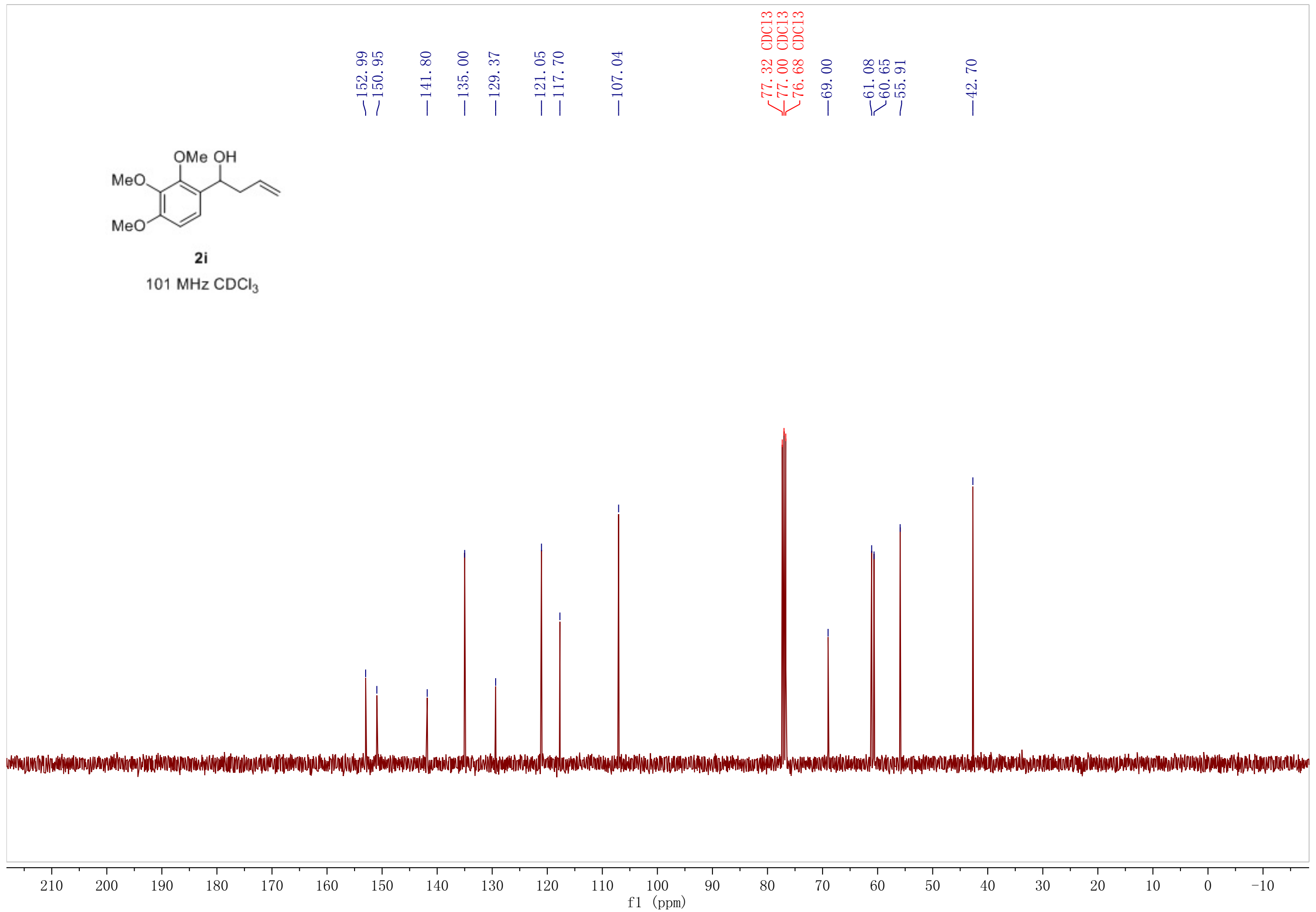




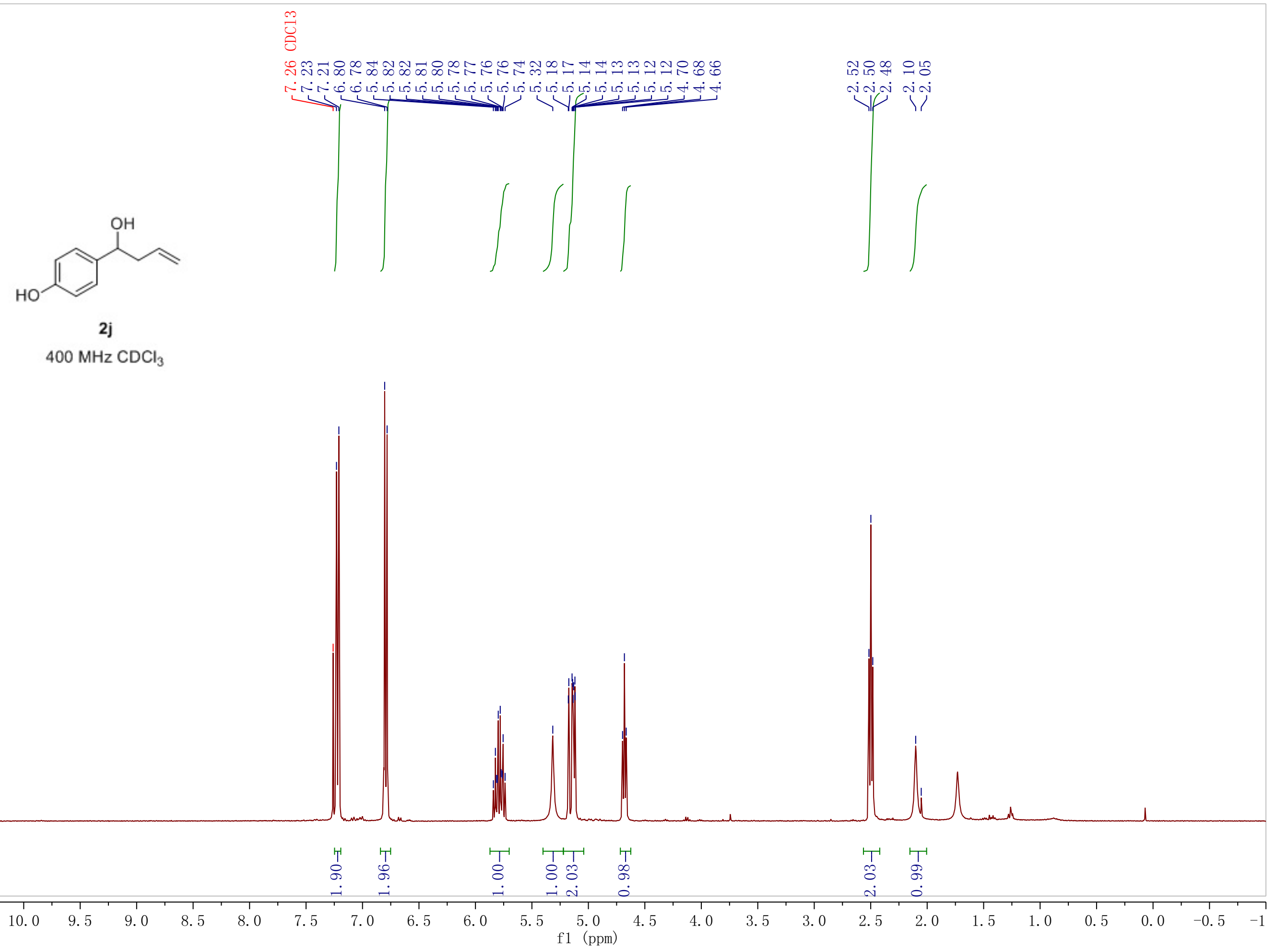




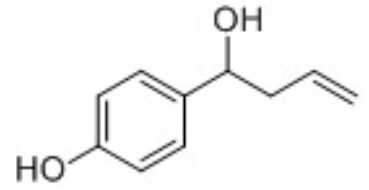

2j

$101 \mathrm{MHz} \mathrm{CDCl}_{3}$

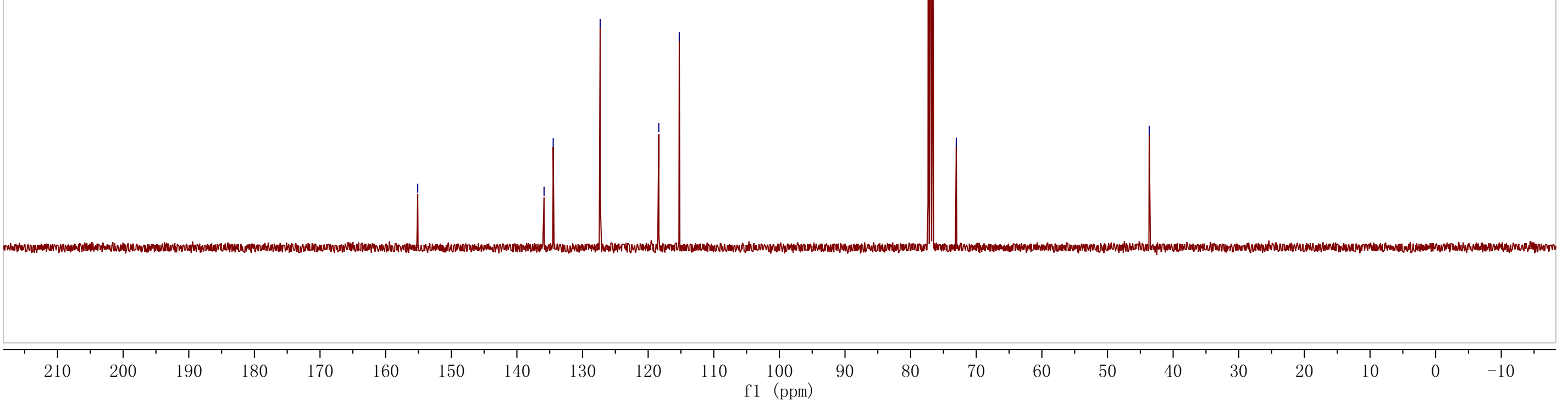




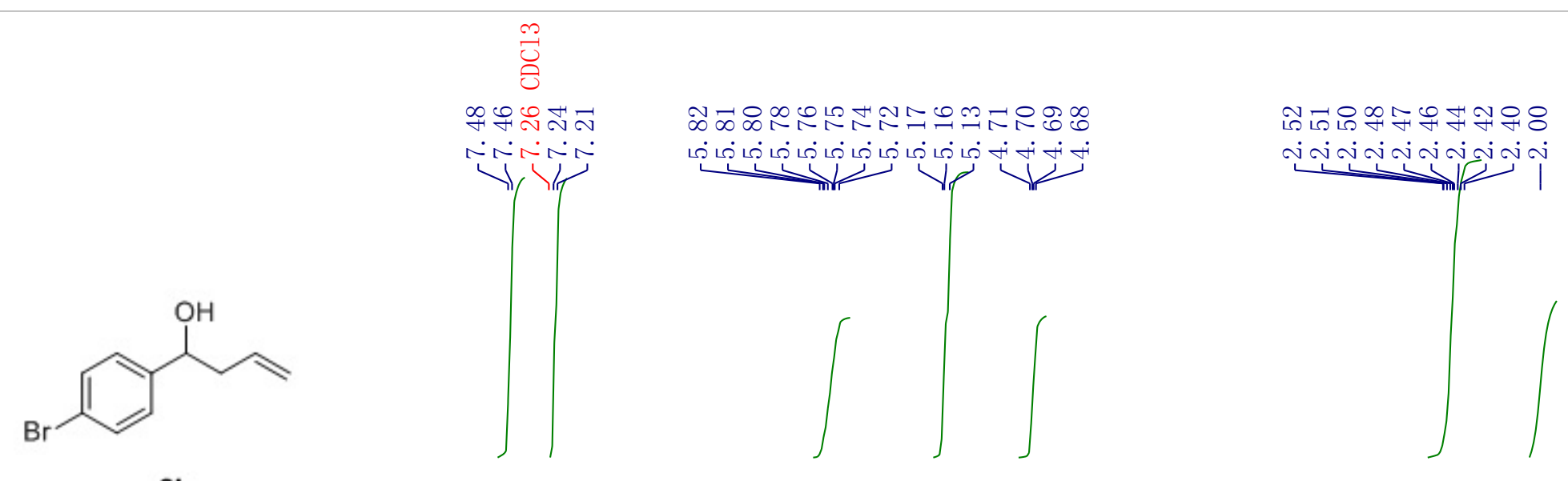

$400 \mathrm{MHz} \mathrm{CDCl}_{3}$

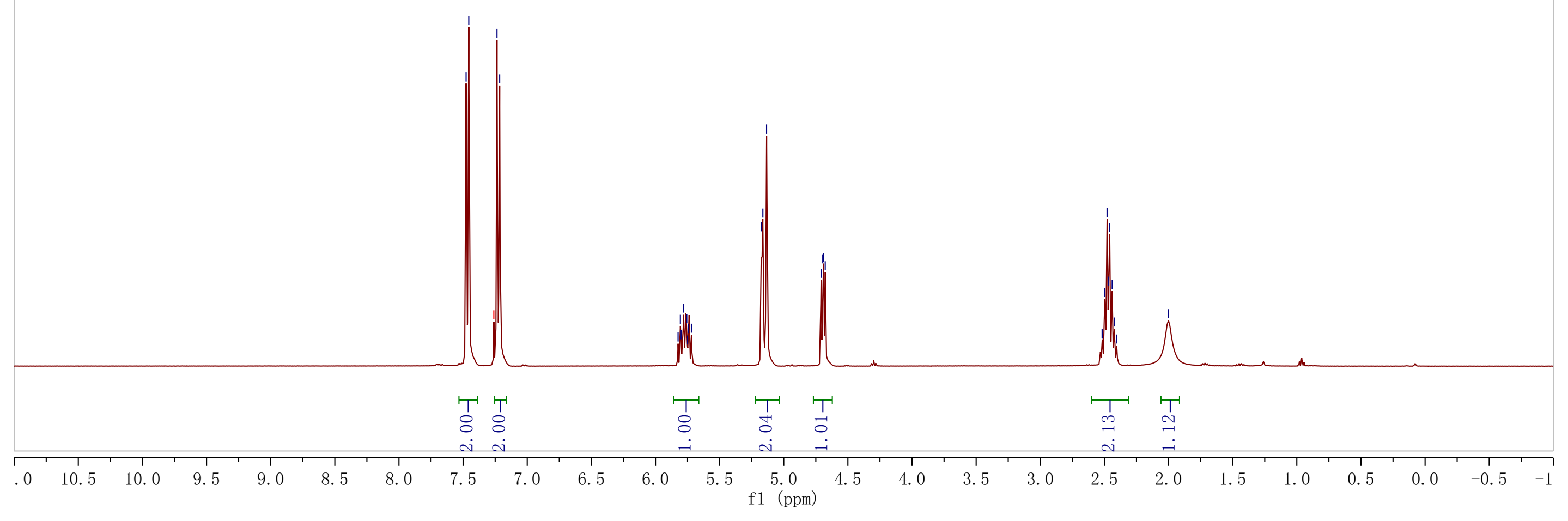




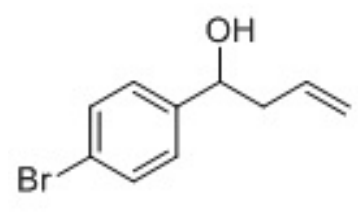

2k

$101 \mathrm{MHz} C D C l_{3}$ 


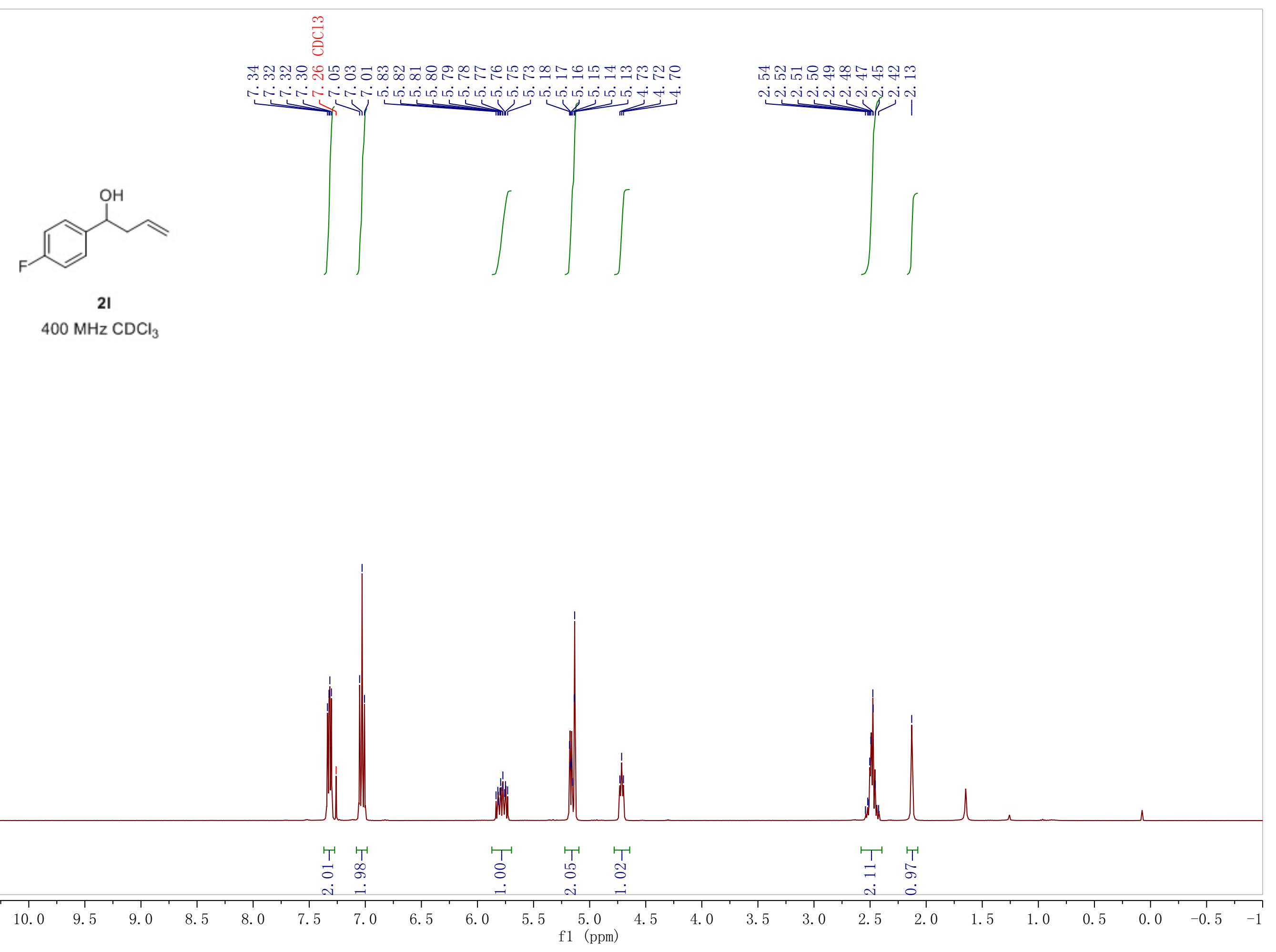




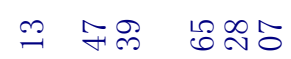

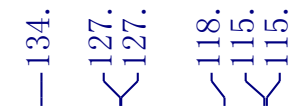

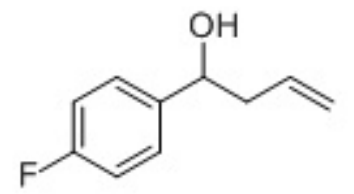

21

$101 \mathrm{MHz} \mathrm{CDCl}_{3}$

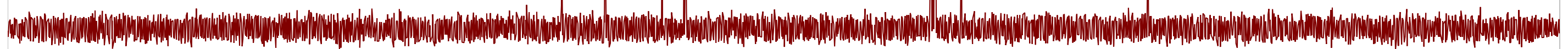

\begin{tabular}{|c|c|c|c|c|c|c|c|c|c|c|c|c|c|c|c|c|c|c|c|c|c|c|}
\hline 210 & 200 & $\begin{array}{c}1 \\
190\end{array}$ & $\begin{array}{l} \\
180\end{array}$ & 170 & $\begin{array}{c}1 \\
160\end{array}$ & $\begin{array}{c}1 \\
150\end{array}$ & 140 & $\begin{array}{l}-1 \\
130\end{array}$ & $\begin{array}{c}1 \\
120\end{array}$ & 110 & $\begin{array}{c}100 \\
\text { f1 }(p p m)\end{array}$ & $\begin{array}{l}1 \\
90\end{array}$ & $\begin{array}{l}1 \\
80\end{array}$ & 70 & 60 & 50 & 40 & $\begin{array}{l}1 \\
30\end{array}$ & 20 & 10 & $\begin{array}{l}1 \\
0\end{array}$ & $\begin{array}{l} \\
-10\end{array}$ \\
\hline
\end{tabular}


டு

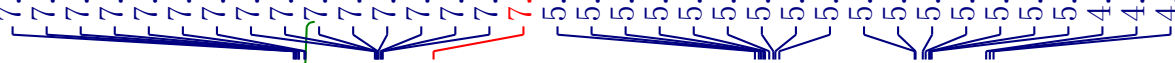

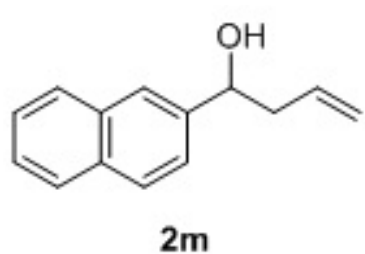

$400 \mathrm{MHz} \mathrm{CDCl}_{3}$

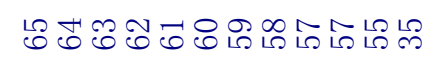

Ninnininivini

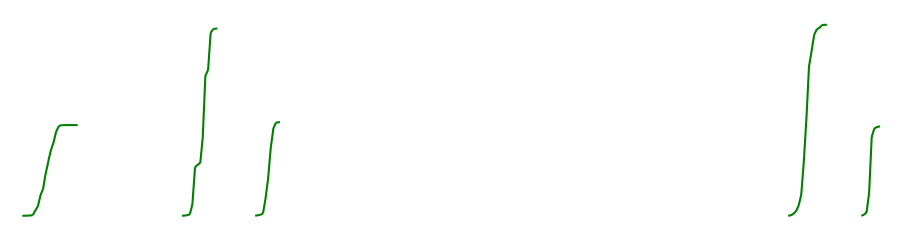

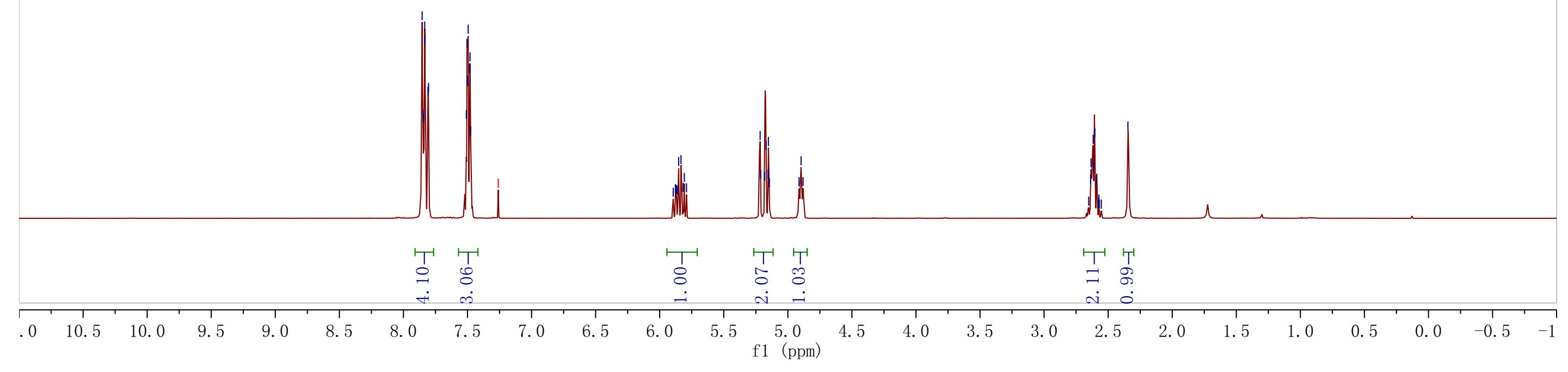




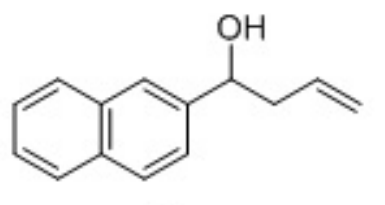

$2 \mathrm{~m}$

$101 \mathrm{MHz} \mathrm{CDCl}_{3}$ 


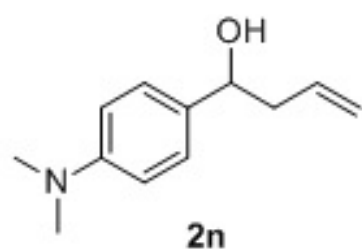

$400 \mathrm{MHz} C D C l_{3}$
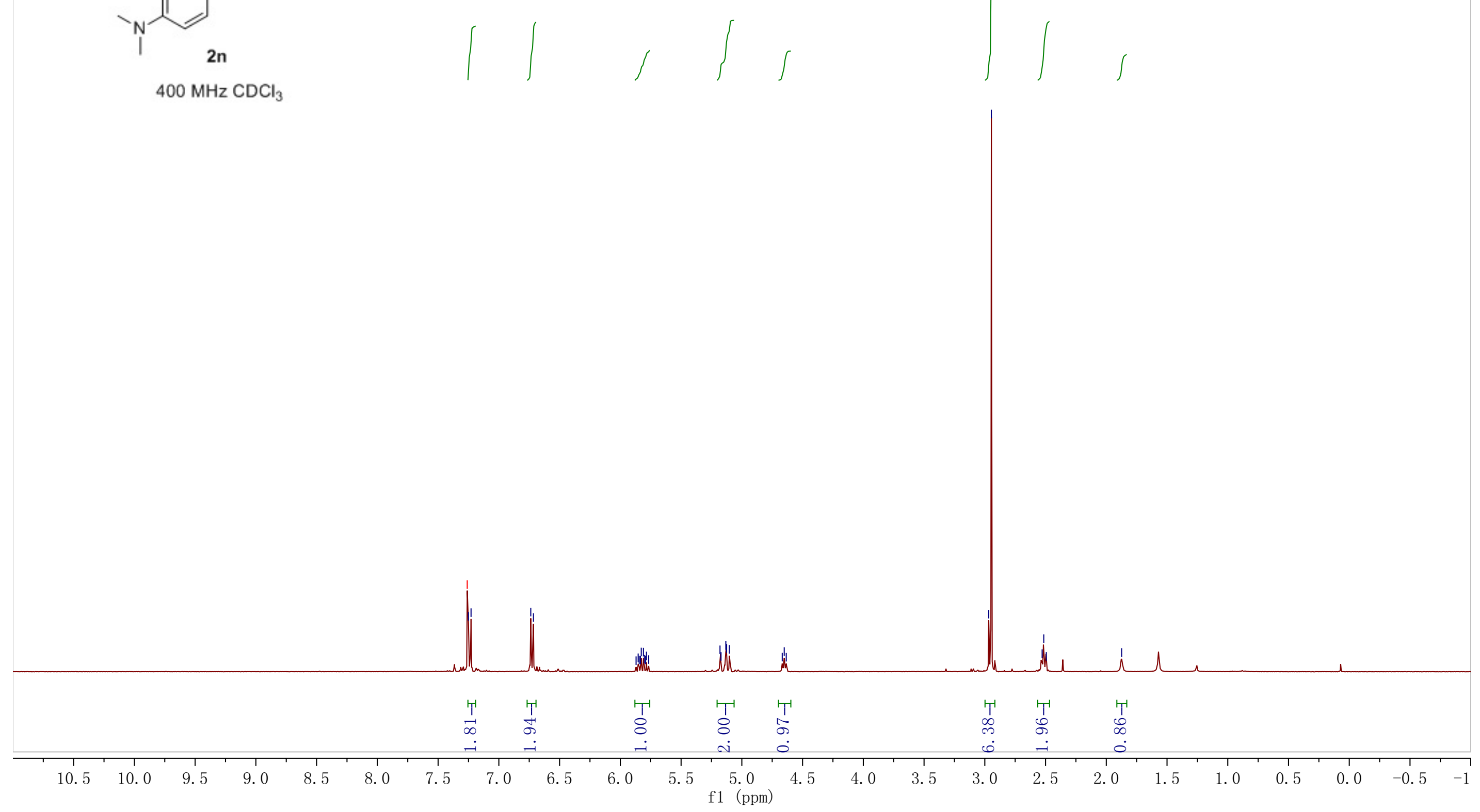


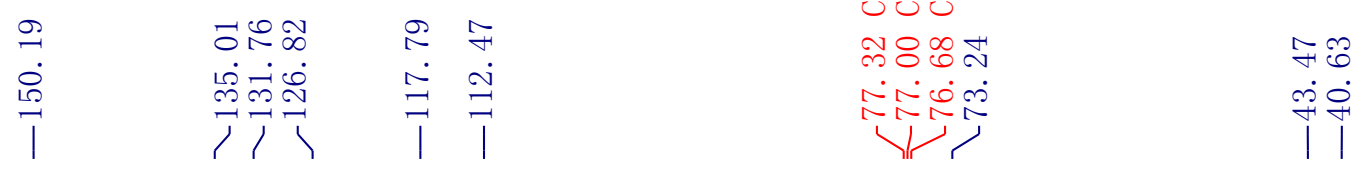

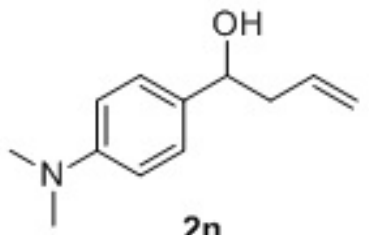

$101 \mathrm{MHz} \mathrm{CDCl}_{3}$

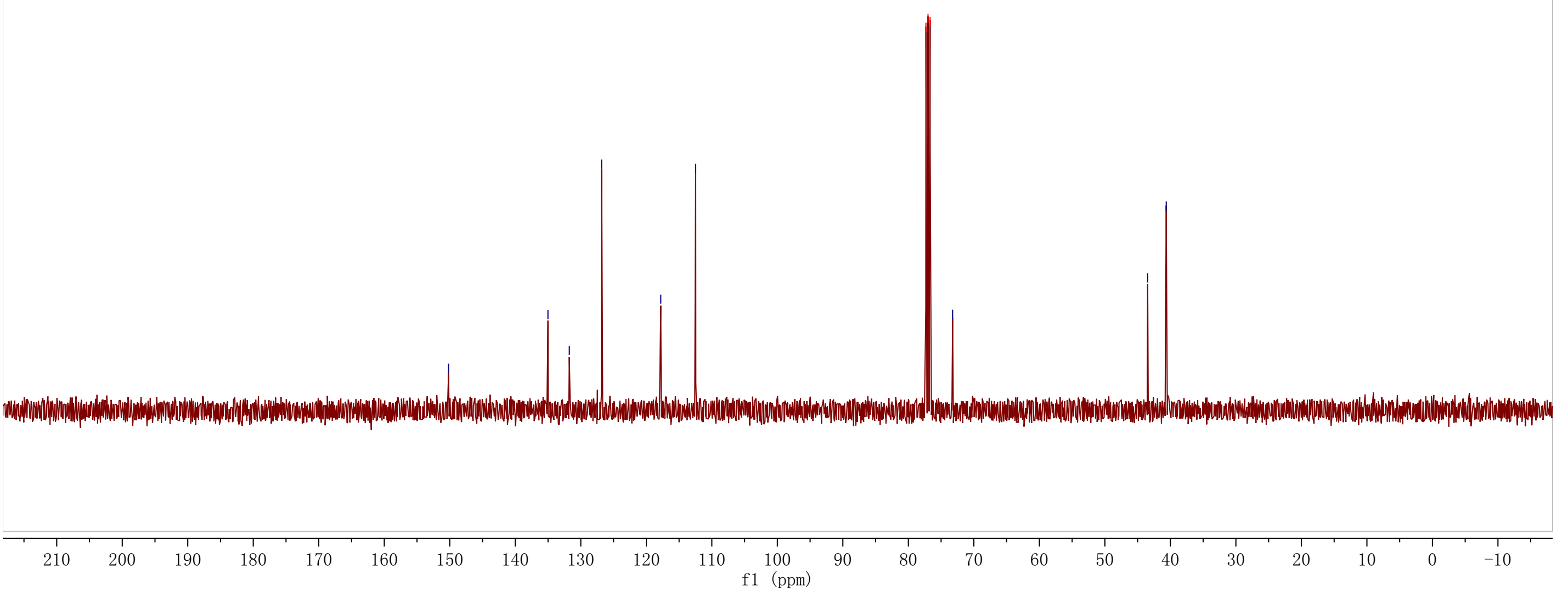




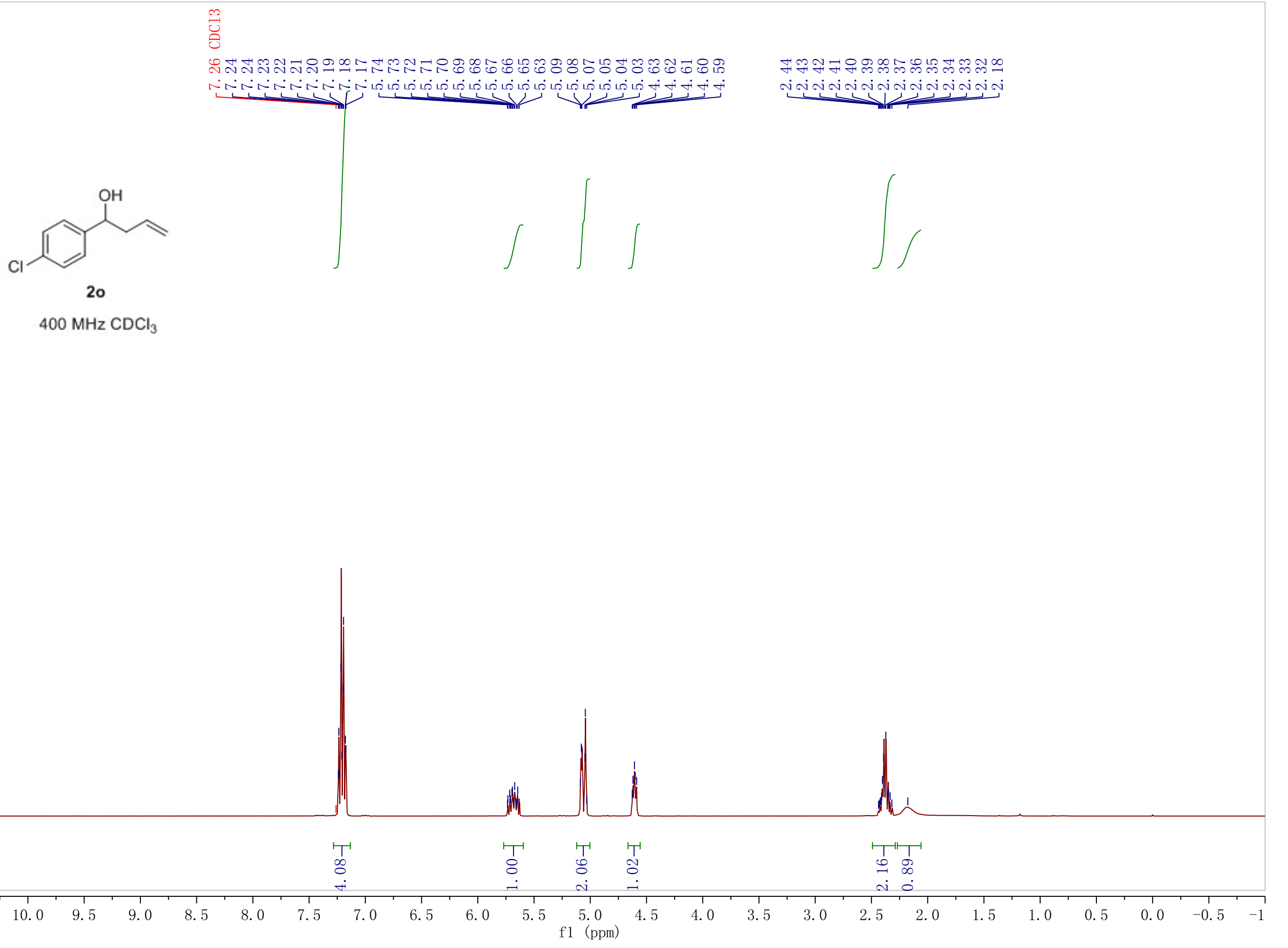




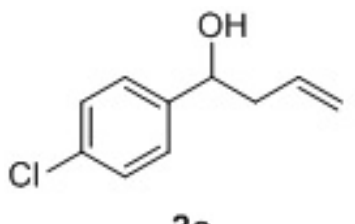

2o

$101 \mathrm{MHz} \mathrm{CDCl}_{3}$

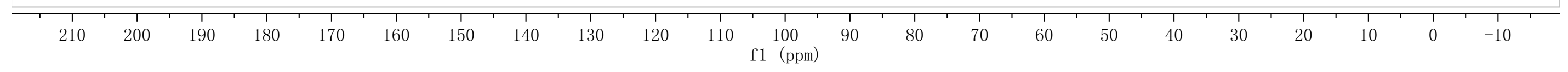




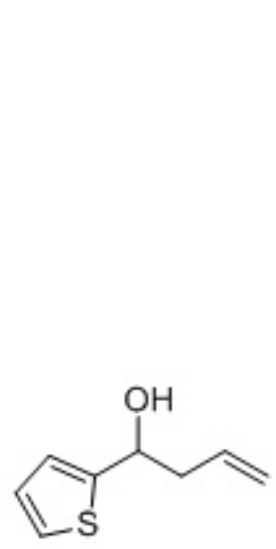

๓

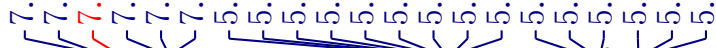

\section{도요}

$\rightarrow$ C

\section{งivis}

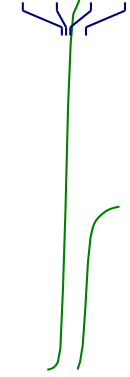

2p

$400 \mathrm{MHz} C D C l_{3}$

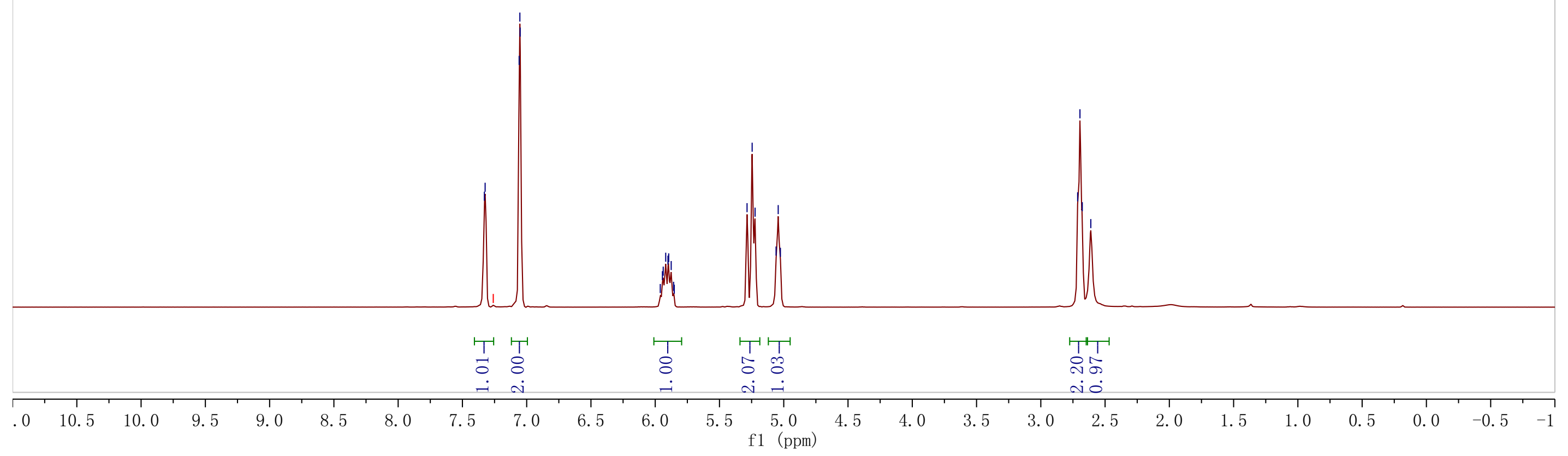




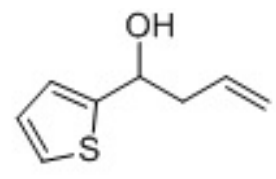

2p

$101 \mathrm{MHz} \mathrm{CDCl}_{3}$

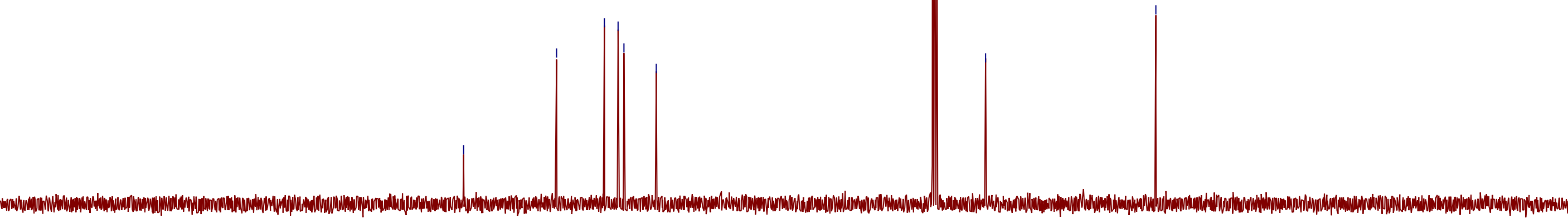

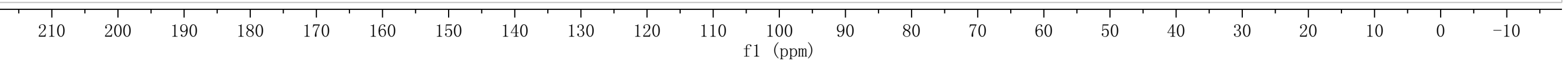




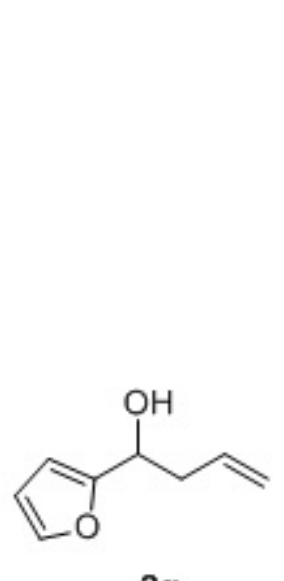

$2 q$ $400 \mathrm{MHz} \mathrm{CDCl}_{3}$

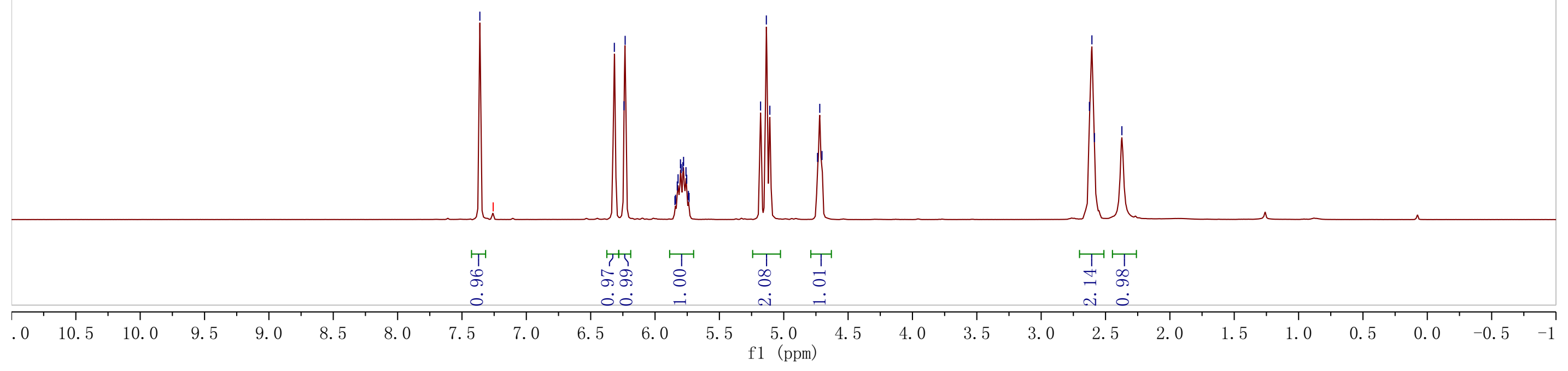




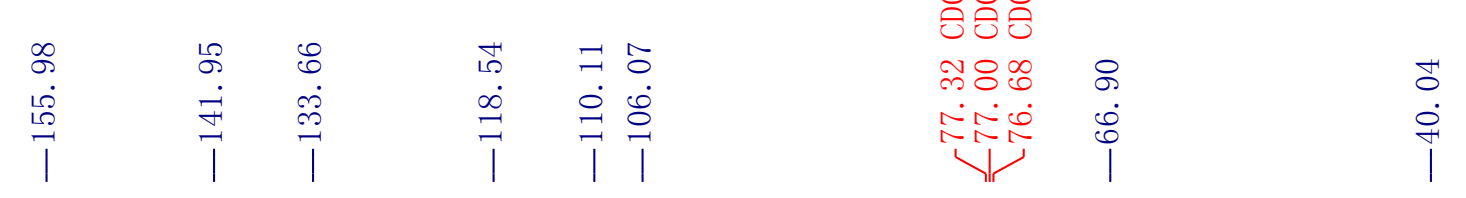

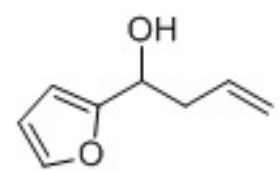

$2 q$

$101 \mathrm{MHz} \mathrm{CDCl}_{3}$

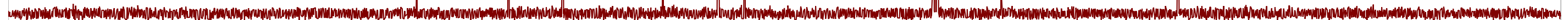

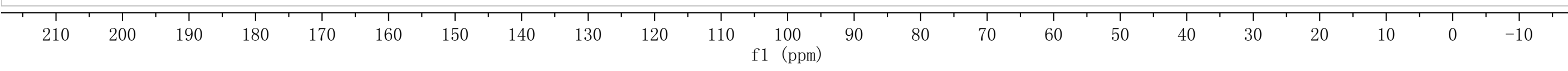




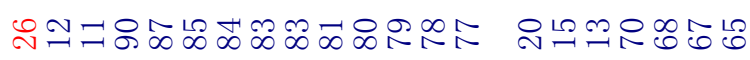

ن

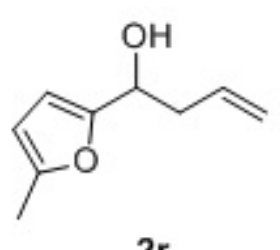

$400 \mathrm{MHz} \mathrm{CDCl}_{3}$
ชิ

نูivinivivi

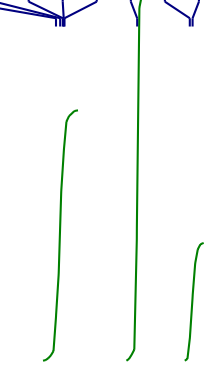

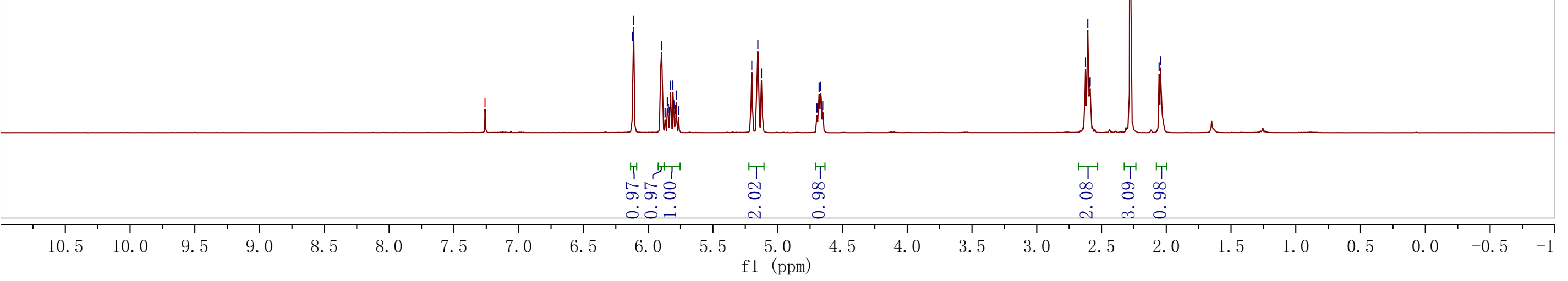




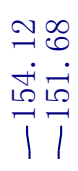

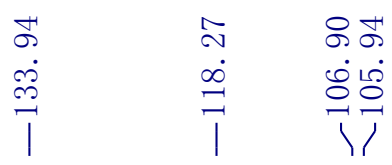

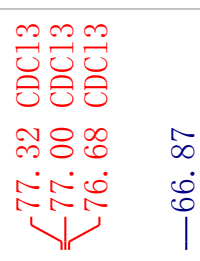

$\dot{5}$
$\infty$
$\dot{P}$

$\stackrel{\infty}{\stackrel{\infty}{!}}$<smiles>C=CCC(O)c1ccc(C)o1</smiles>

$\mathbf{2 r}$
$101 \mathrm{MHz} \mathrm{CDCl}_{3}$

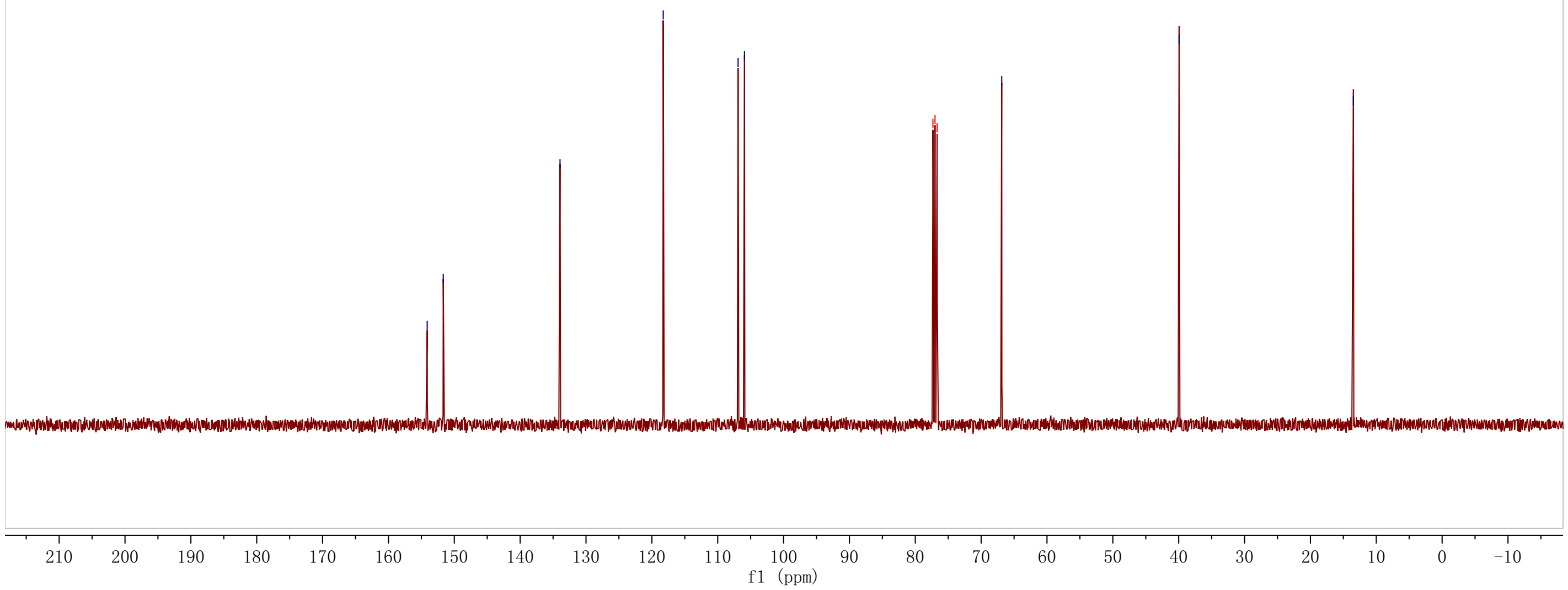




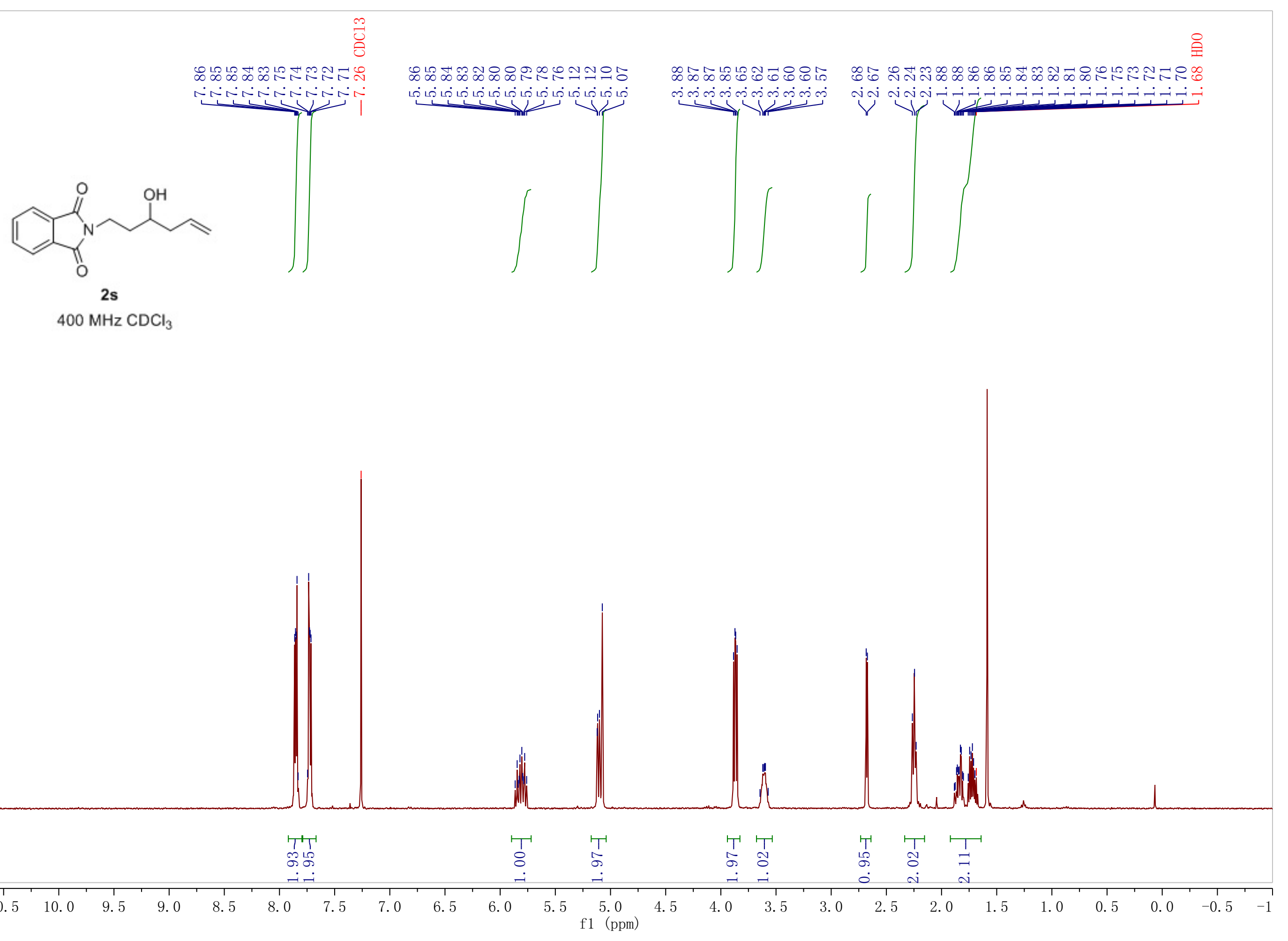




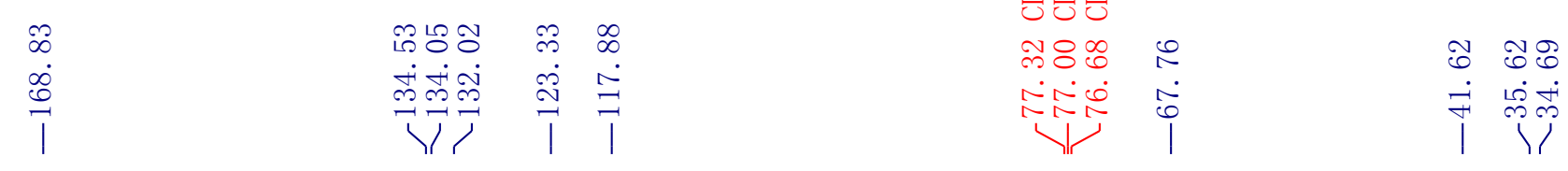

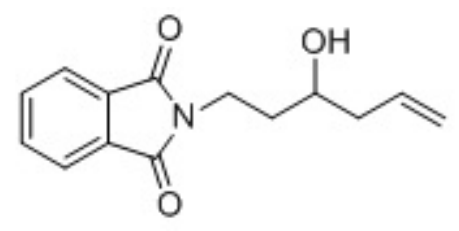

$2 \mathrm{~s}$

$101 \mathrm{MHz} \mathrm{CDCl}_{3}$

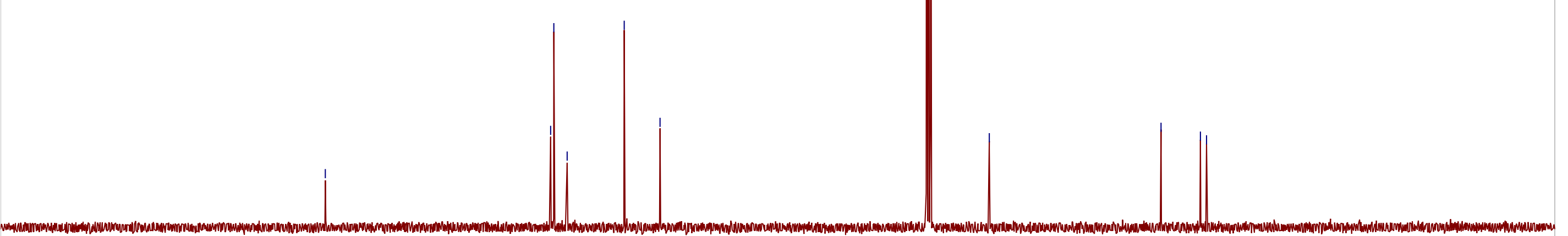




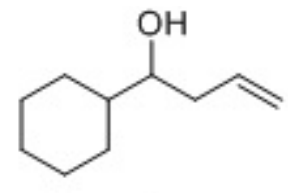

$2 \mathrm{t}$ $400 \mathrm{MHz} C D C l_{3}$

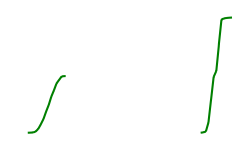

$\int$

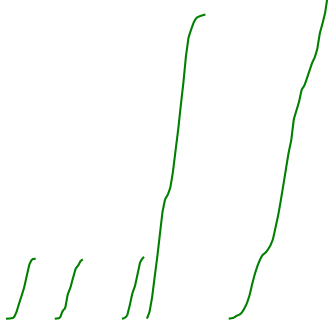

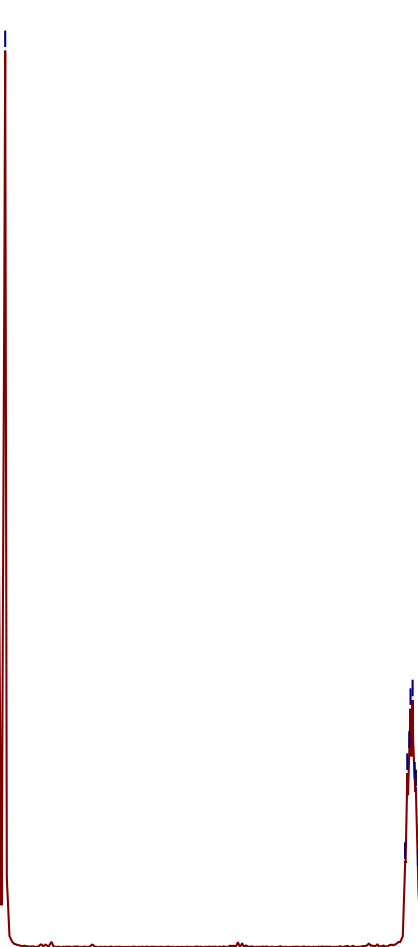
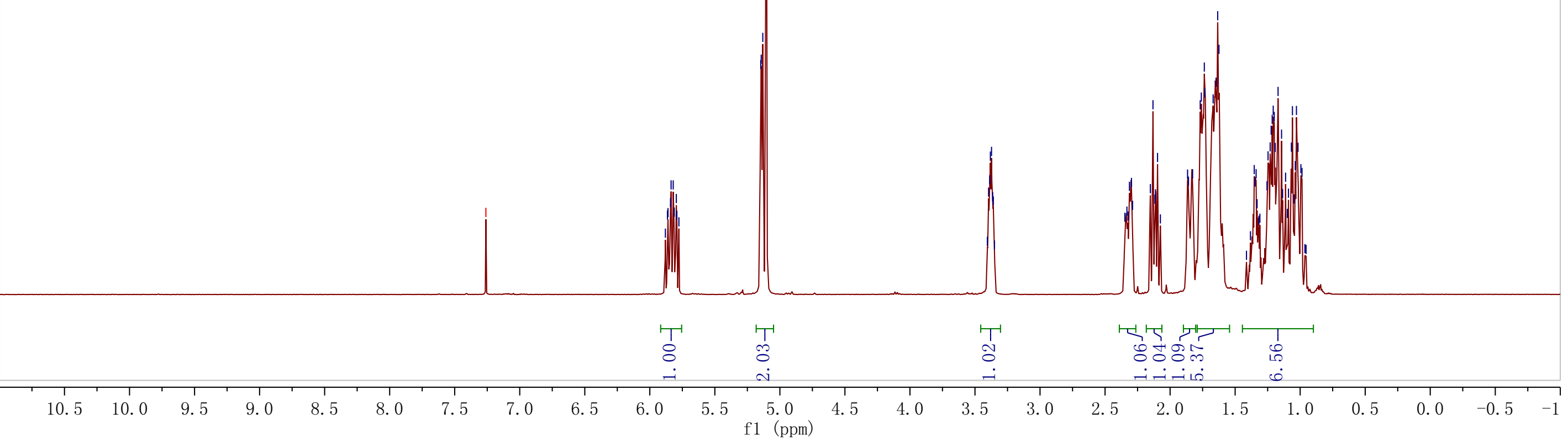


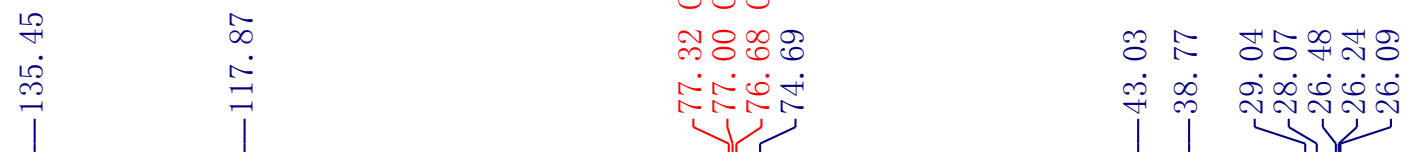

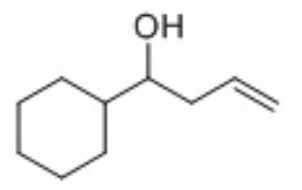

$2 \mathrm{t}$

$101 \mathrm{MHz} \mathrm{CDCl}_{3}$

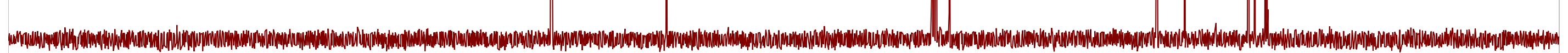

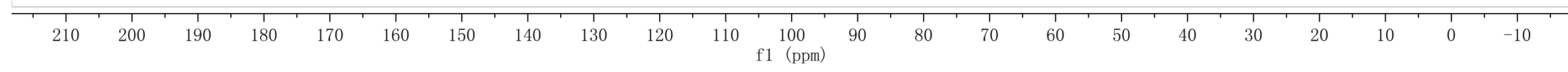




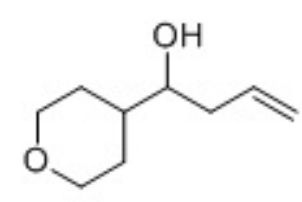

2u

$400 \mathrm{MHz} \mathrm{CDCl}_{3}$

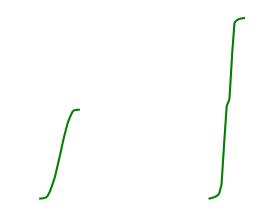

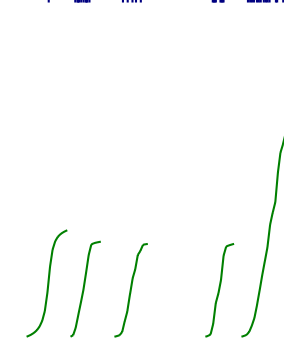

(a)
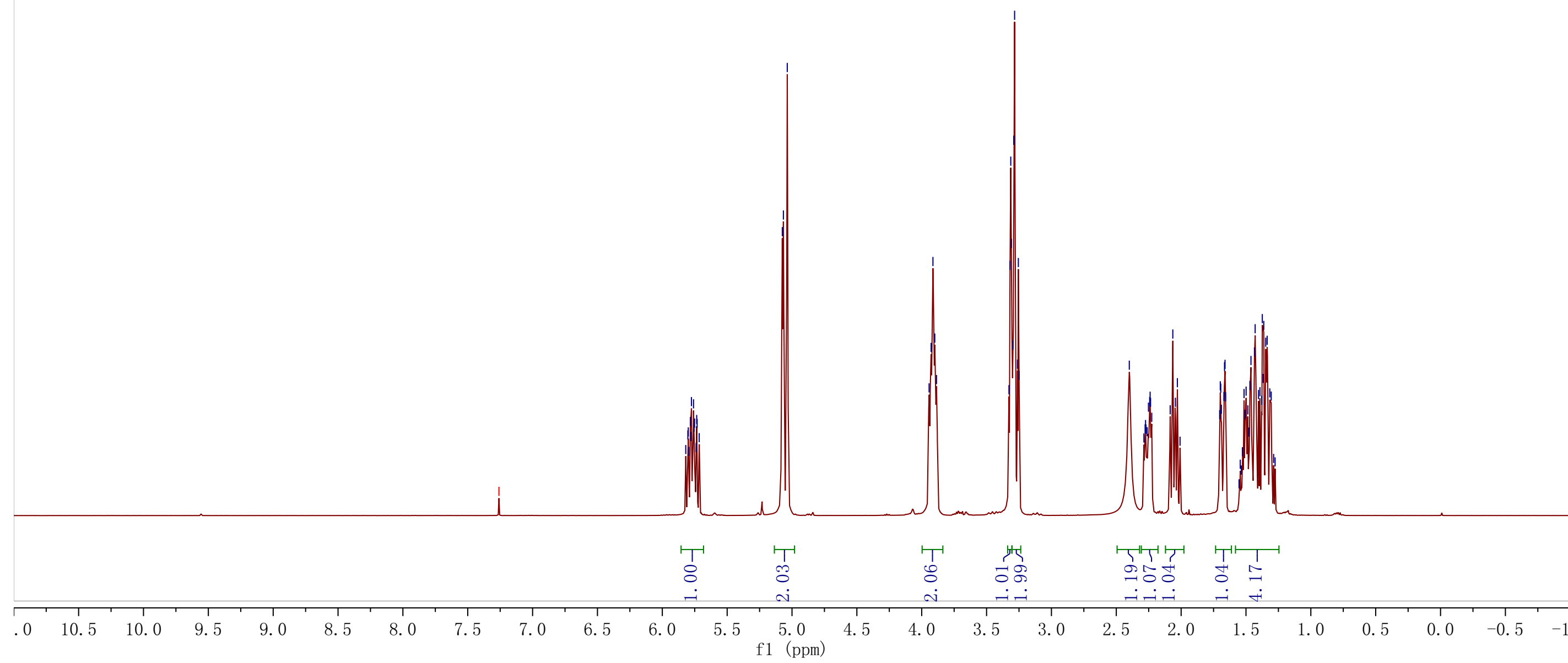


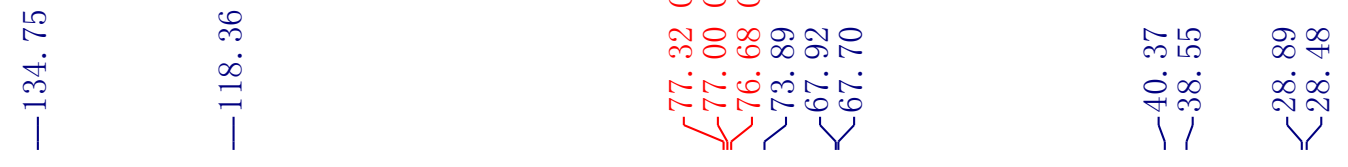

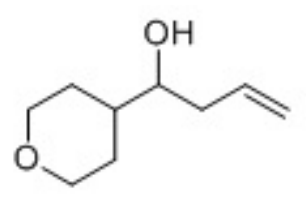

$2 \mathrm{u}$

$101 \mathrm{MHz} \mathrm{CDCl}_{3}$

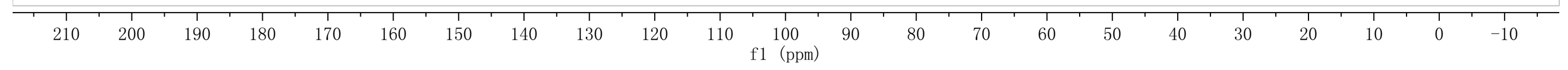




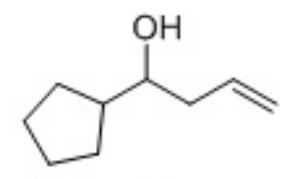

$2 \mathrm{v}$

$400 \mathrm{MHz} \mathrm{CDCl}_{3}$

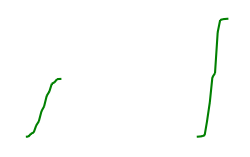

$\int$

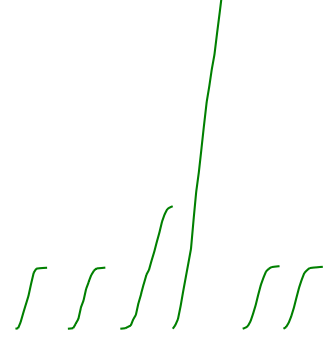

(2)

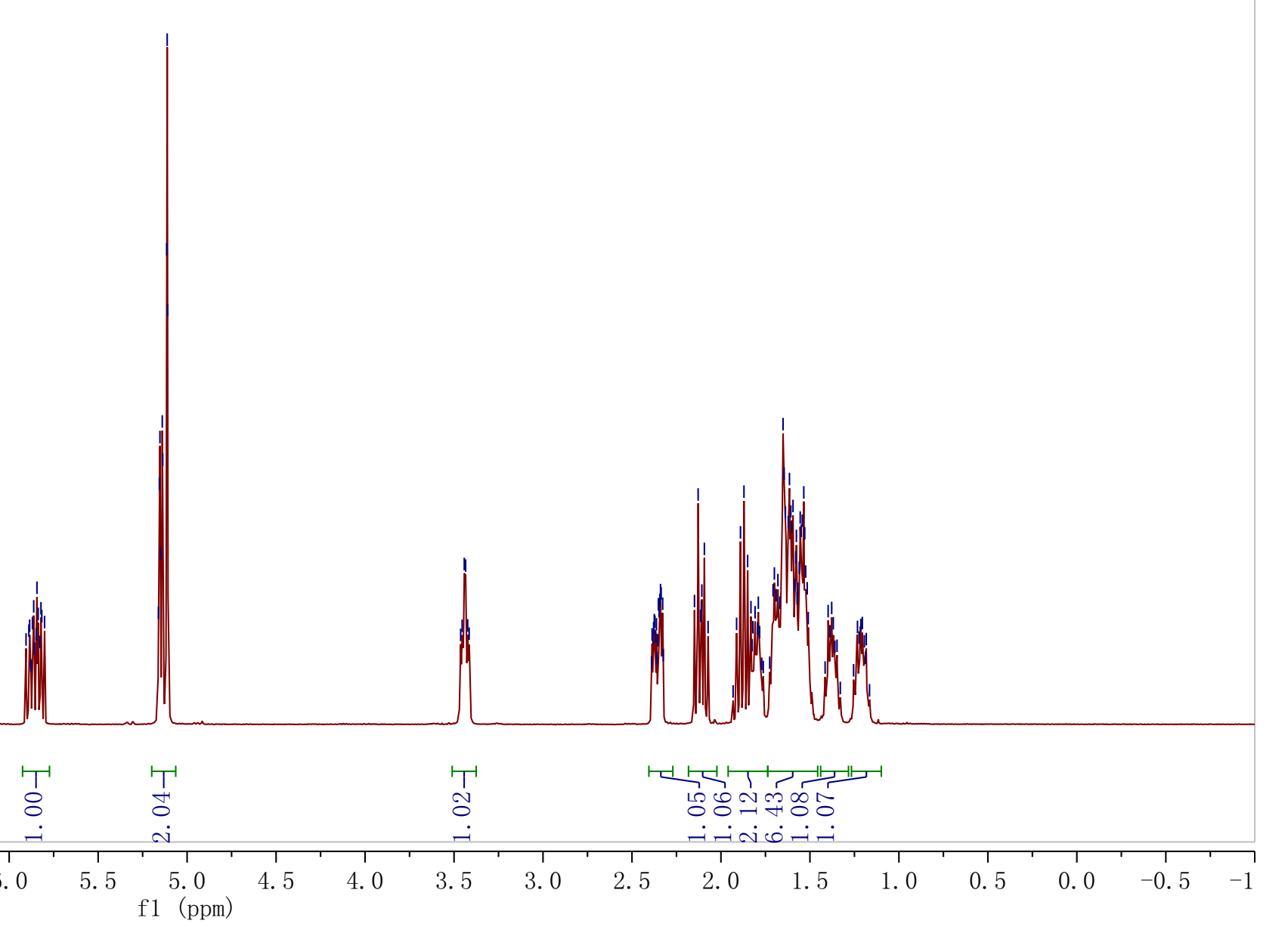




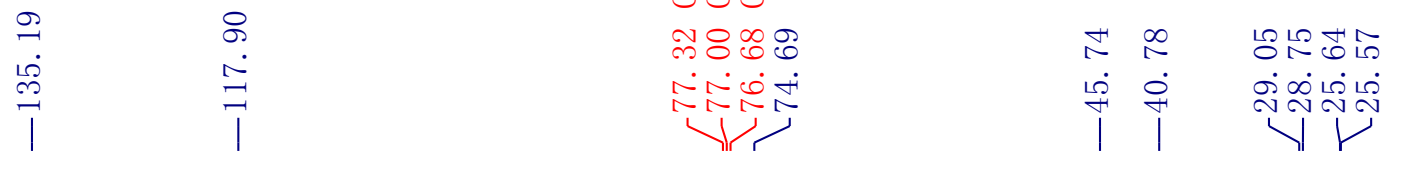

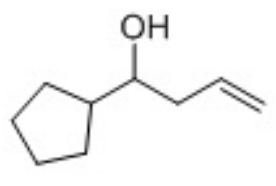

2v

$101 \mathrm{MHz} \mathrm{CDCl}_{3}$

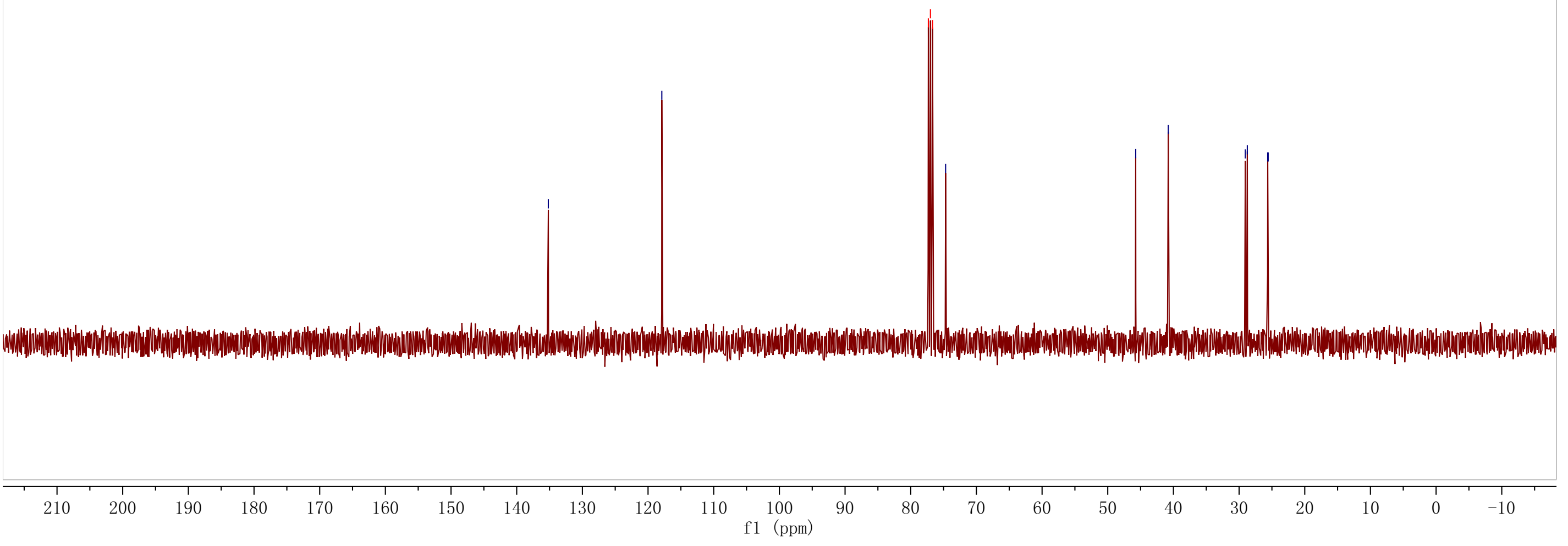



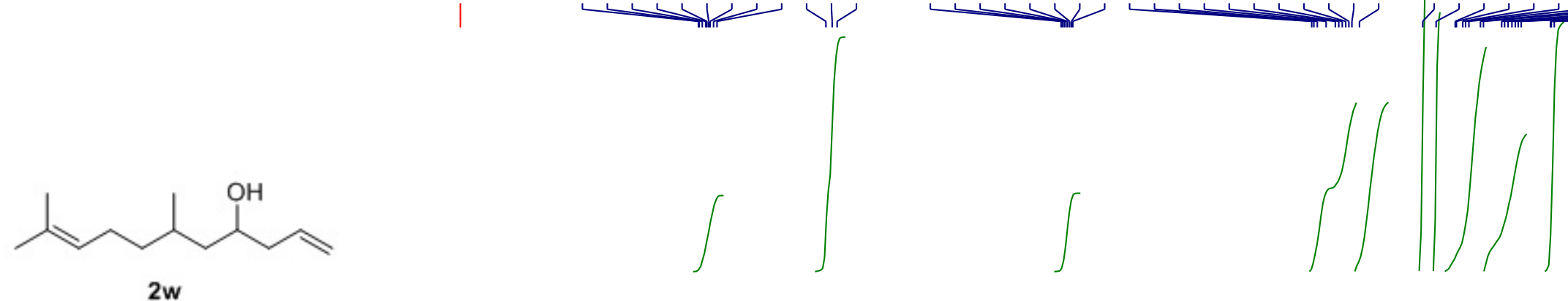

$400 \mathrm{MHz} \mathrm{CDCl}_{3}$

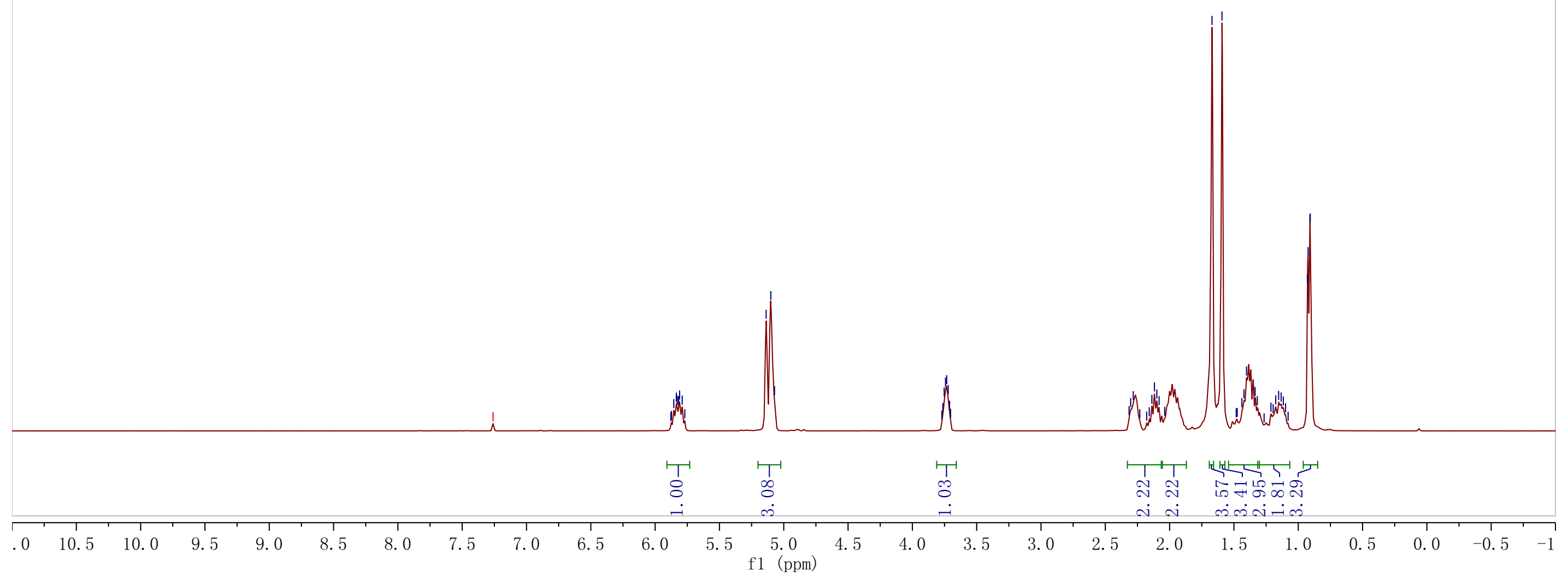




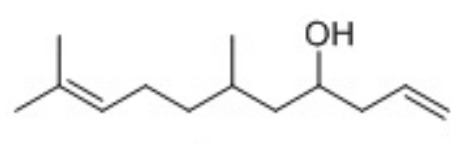

$2 \mathrm{w}$

$101 \mathrm{MHz} \mathrm{CDCl}_{3}$

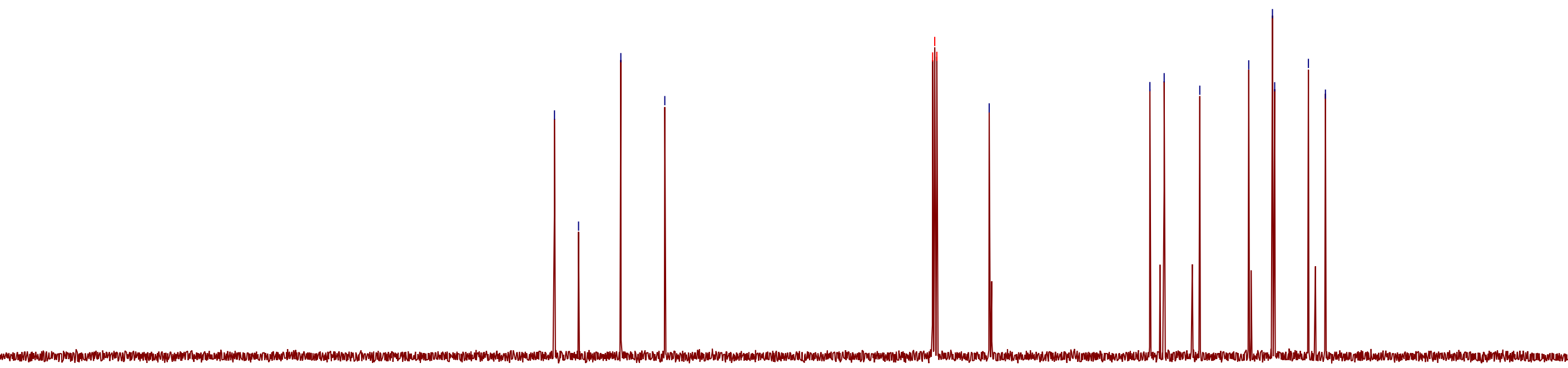

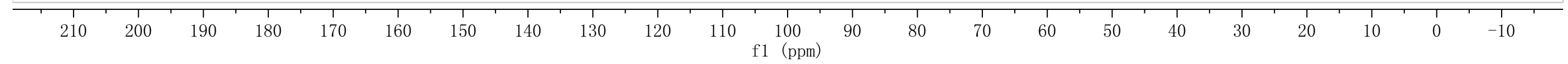



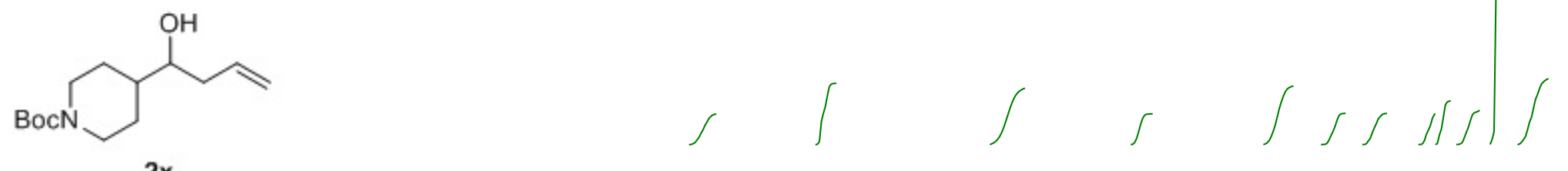

$400 \mathrm{MHz} \mathrm{CDCl}_{3}$

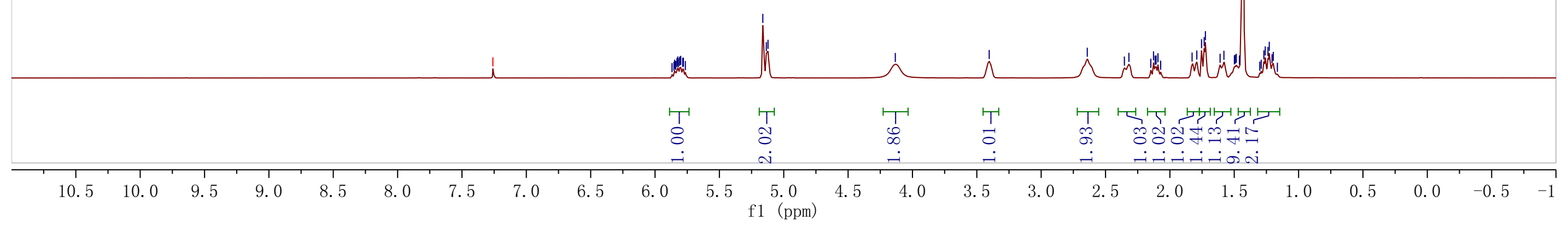




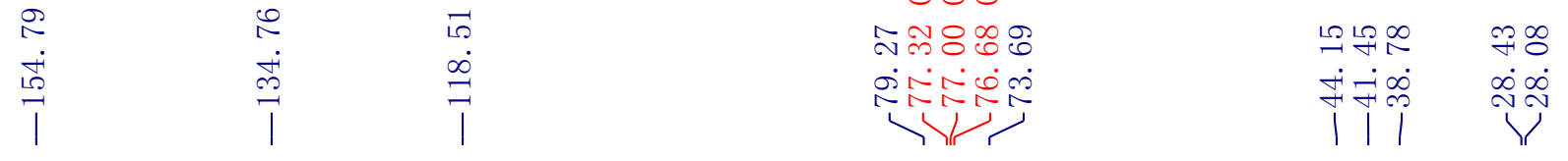

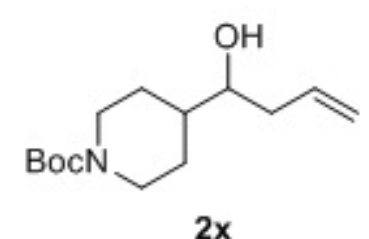

$101 \mathrm{MHz} \mathrm{CDCl}_{3}$

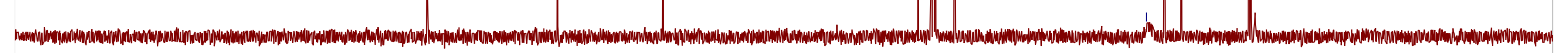

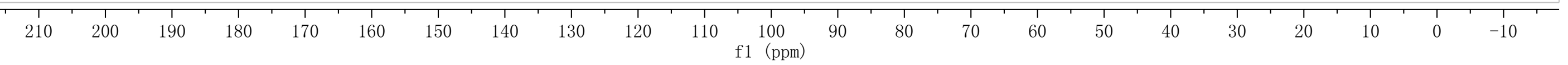



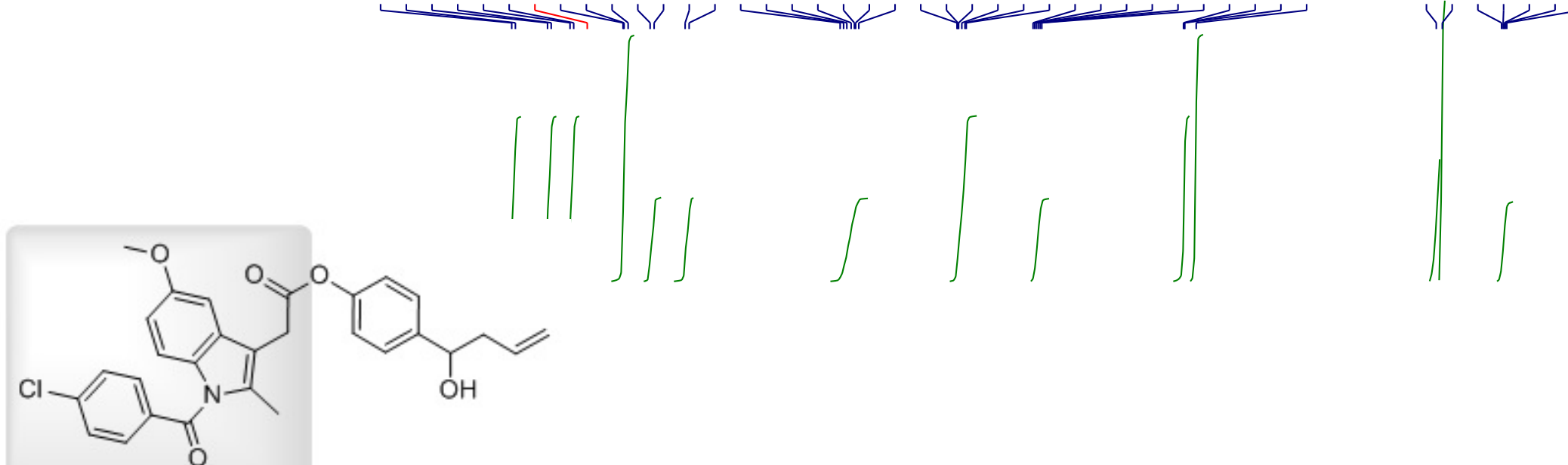

$3 a$

$400 \mathrm{MHz} \mathrm{CDCl}_{3}$

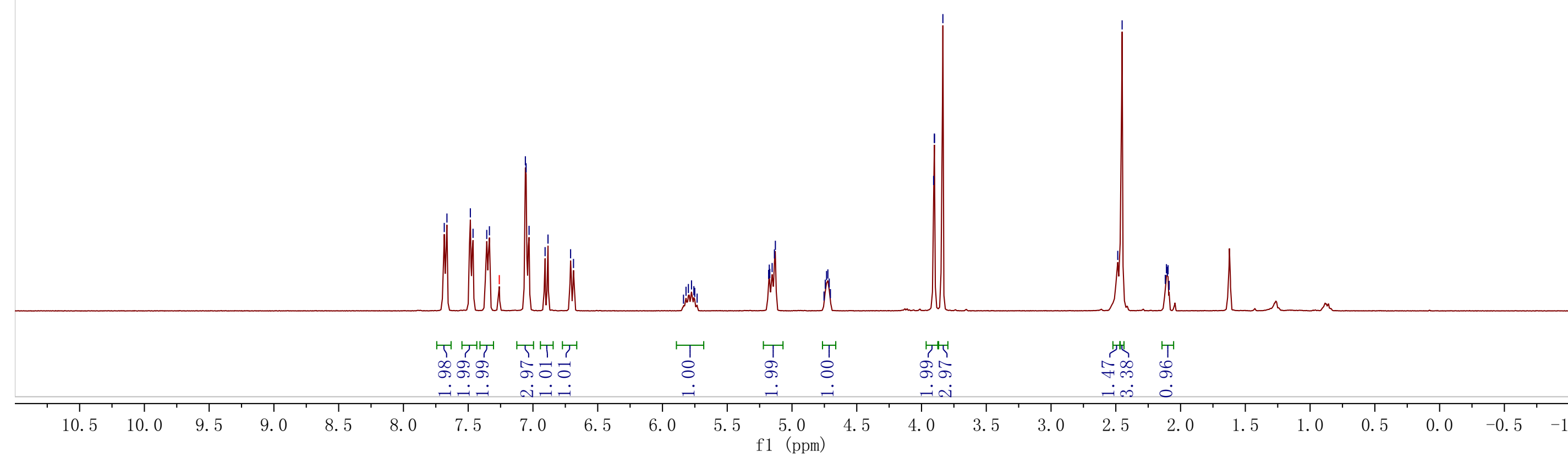


$-0$<smiles>C=CCC(O)c1ccc(OC(=O)Cc2c(C)n(C(=C)C3=CC=C(Cl)CC3)c3ccc(OC)cc23)cc1</smiles>

$3 a$

$101 \mathrm{MHz} \mathrm{CDCl}_{3}$

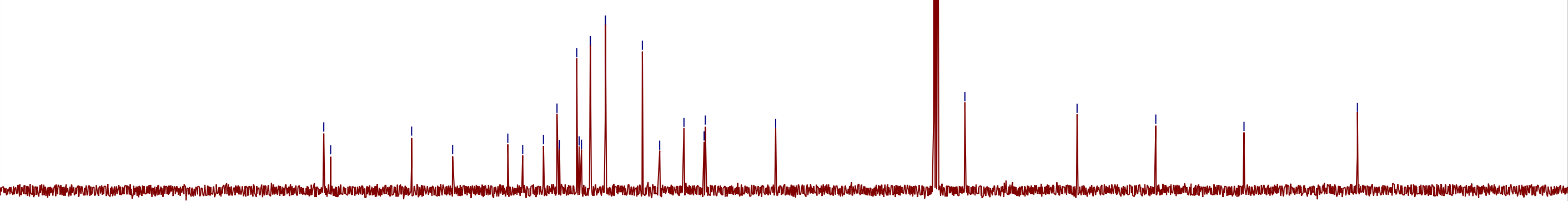



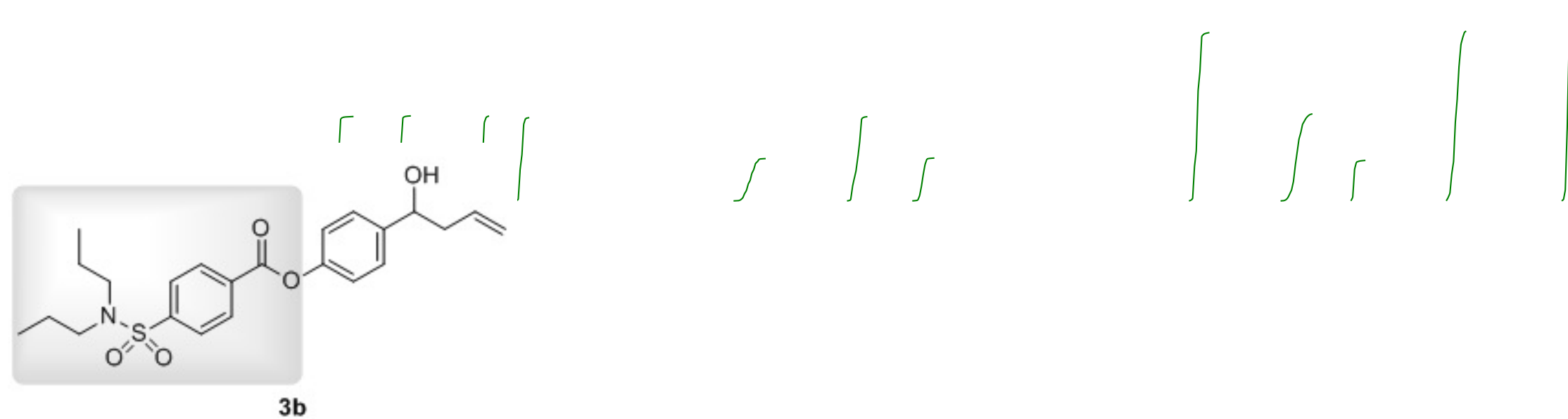

$400 \mathrm{MHz} \mathrm{CDCl}_{3}$

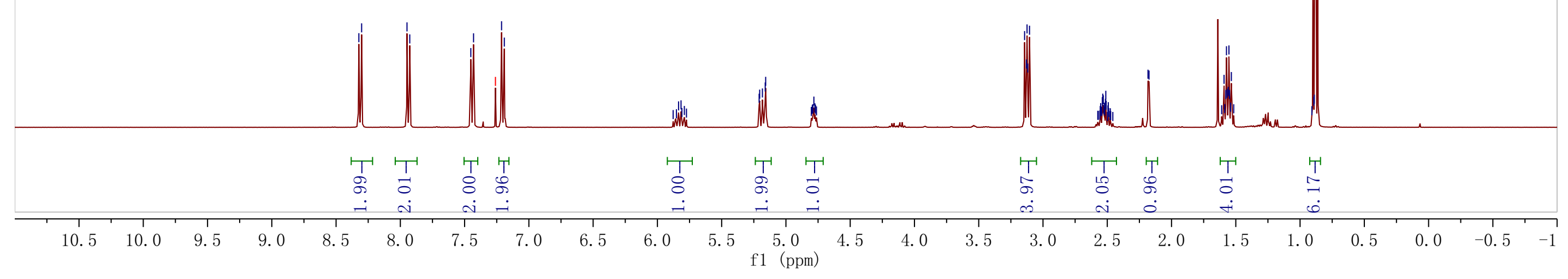




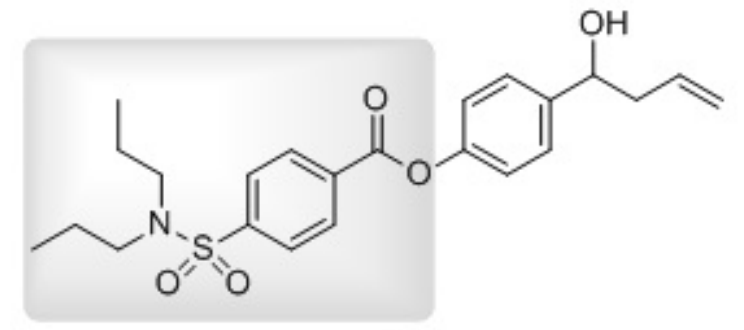

3b

$101 \mathrm{MHz} \mathrm{CDCl}_{3}$

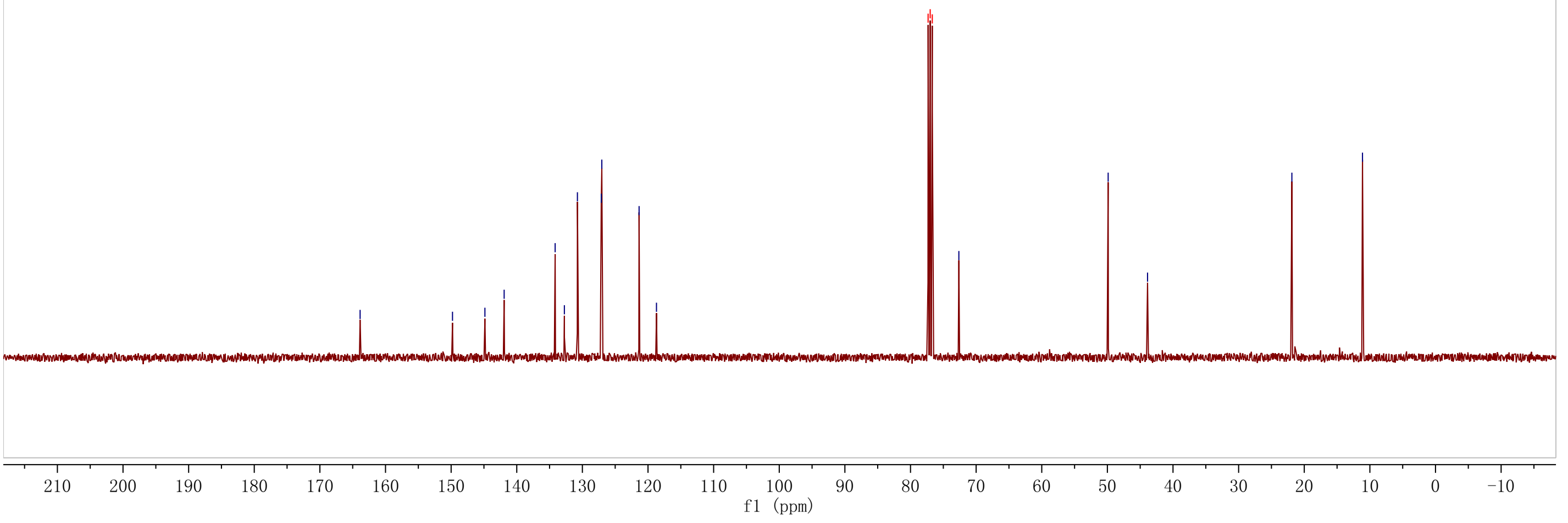



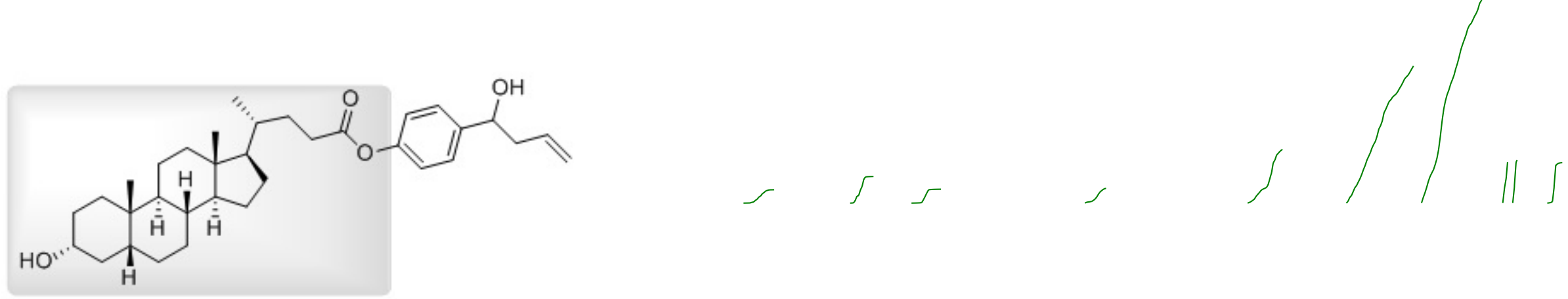

$3 c$

$400 \mathrm{MHz} \mathrm{CDCl}_{3}$

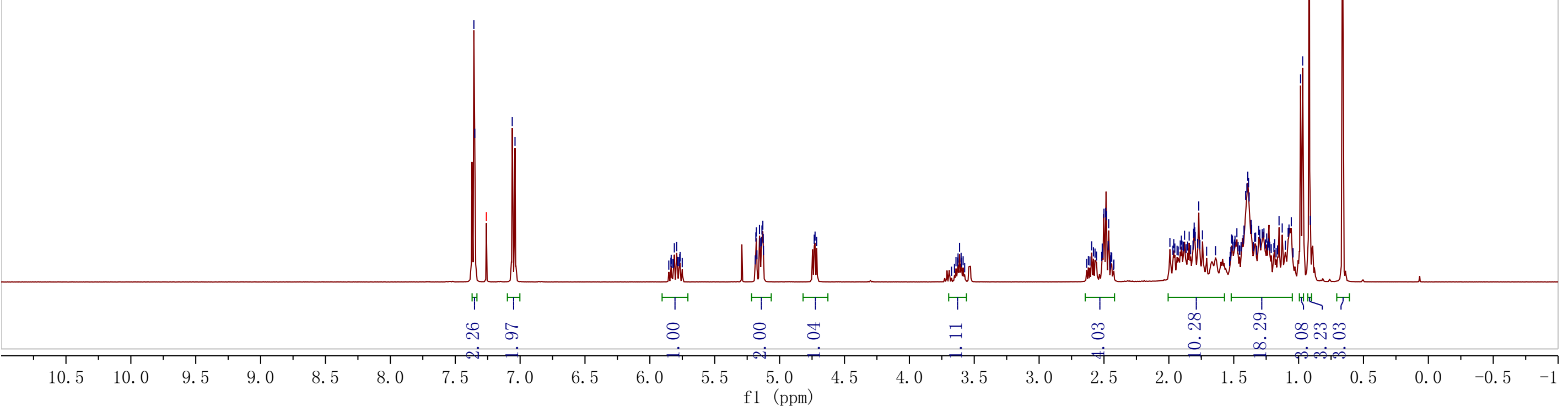




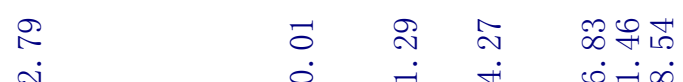 \\ i}

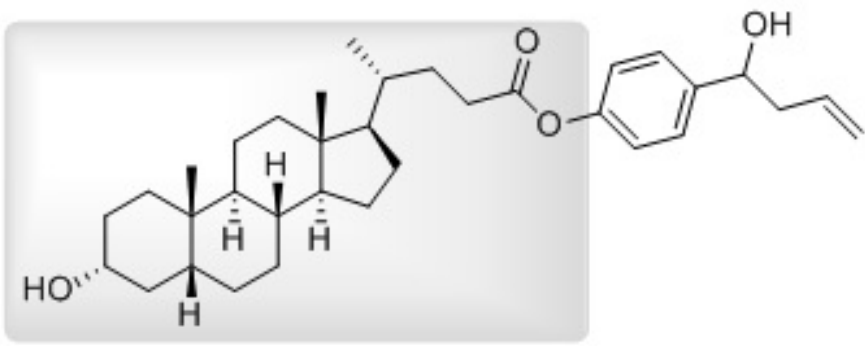

$3 \mathrm{c}$

$101 \mathrm{MHz} \mathrm{CDCl}_{3}$

101 $\mathrm{MHz}_{\mathrm{CDCl}}$

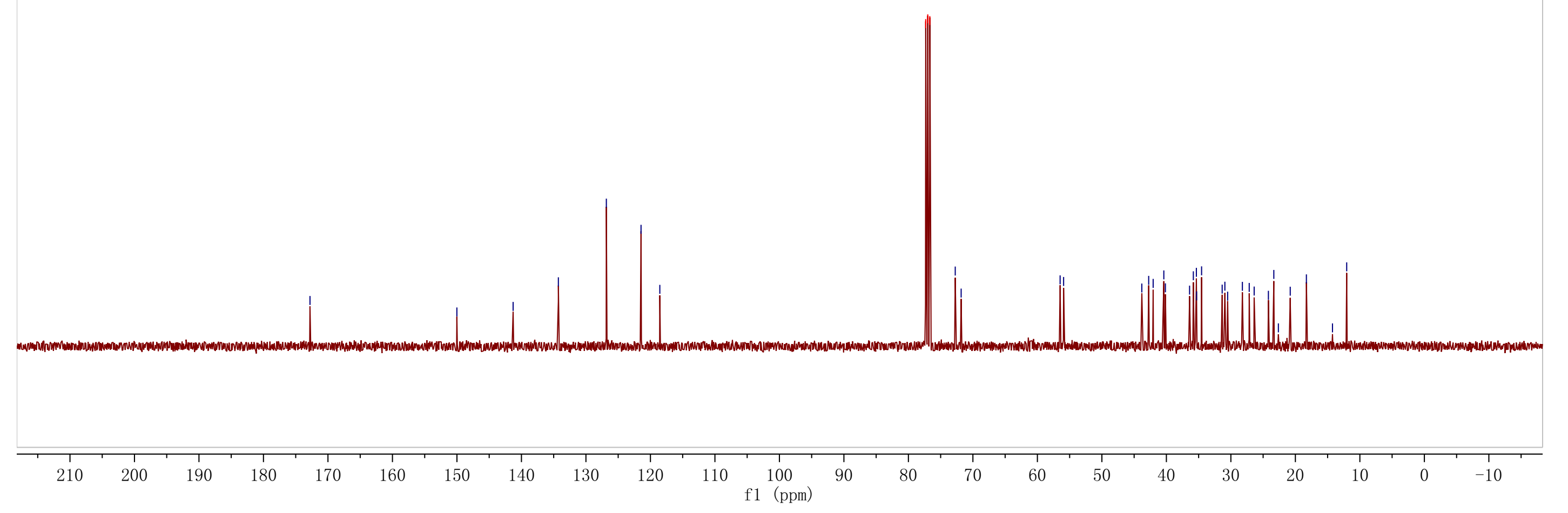




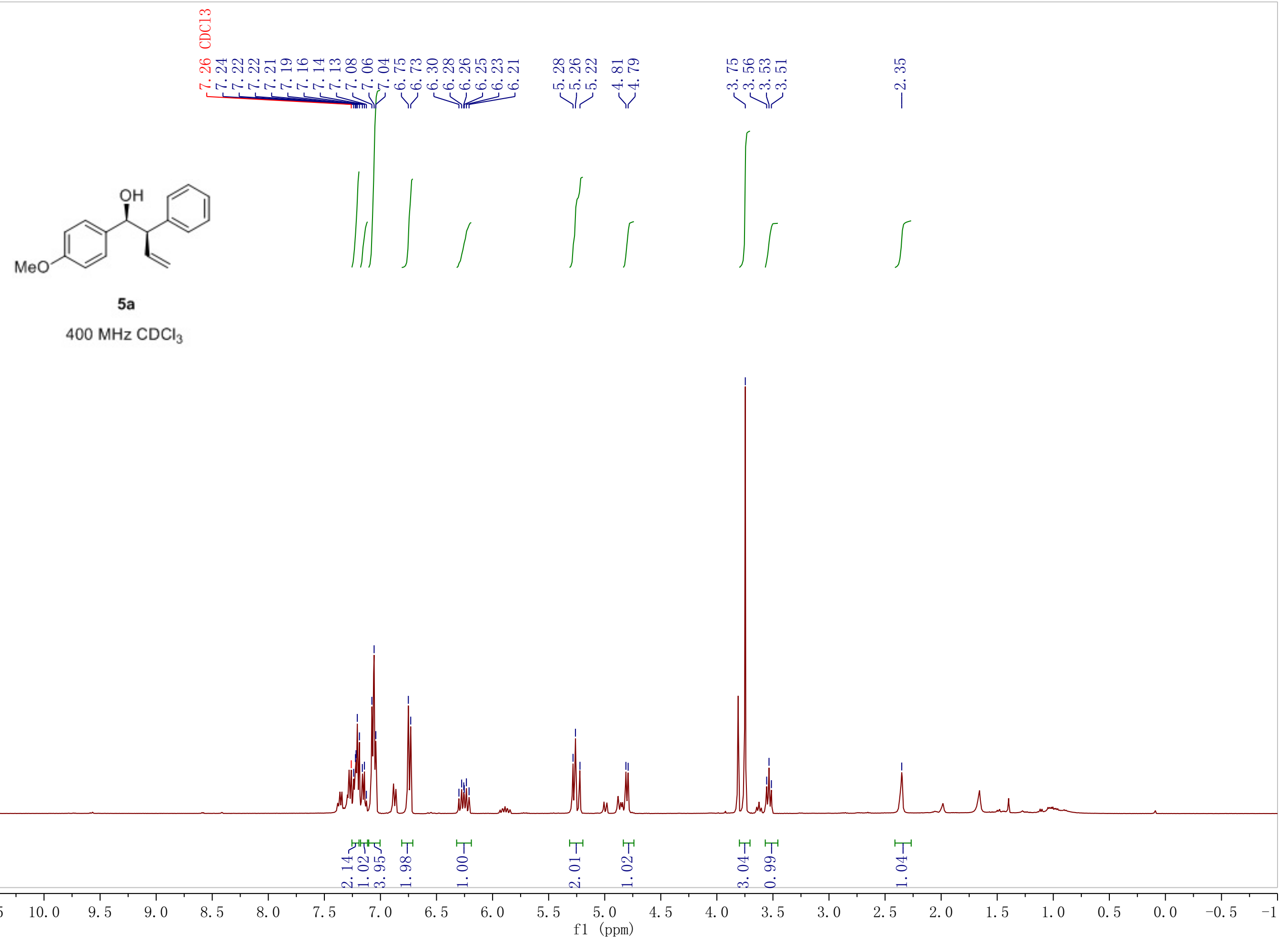




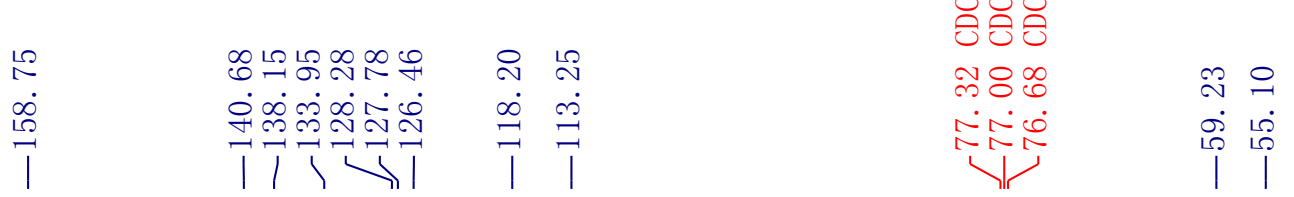

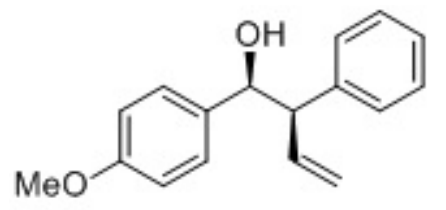

$5 a$

$101 \mathrm{MHz} \mathrm{CDCl}_{3}$

\begin{tabular}{|c|c|c|c|c|c|c|c|c|c|c|c|c|c|c|c|c|c|c|c|c|c|c|}
\hline 210 & 200 & 190 & 180 & 170 & 160 & 150 & 140 & 130 & 120 & 110 & $\begin{array}{c}100 \\
\mathrm{f} 1(\mathrm{ppm})\end{array}$ & 90 & 80 & 70 & 60 & 50 & 40 & 30 & 20 & 10 & 0 & -10 \\
\hline
\end{tabular}


<smiles>C=CC(c1ccccc1)[C@H](O)c1ccccc1-c1ccccc1</smiles>

5b

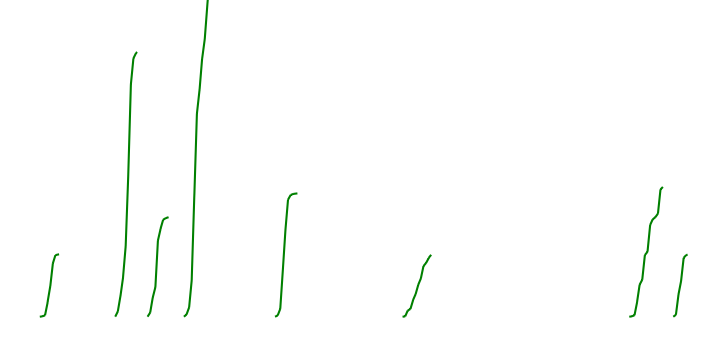

$400 \mathrm{MHz} \mathrm{CDCl}_{3}$
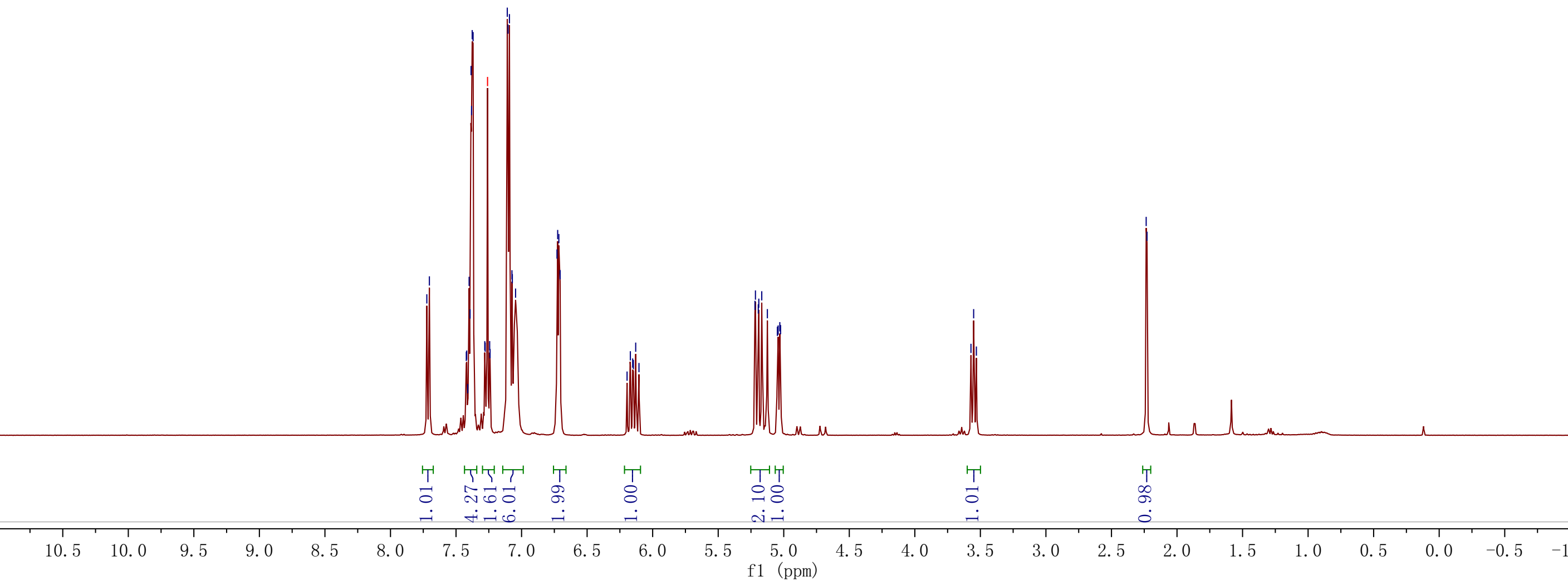


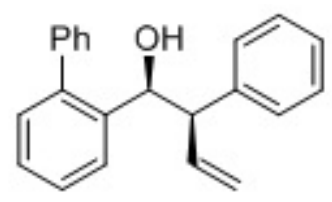

5b

$101 \mathrm{MHz} \mathrm{CDCl}_{3}$

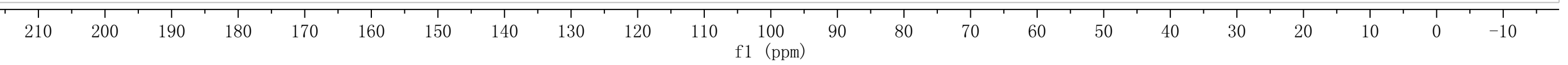




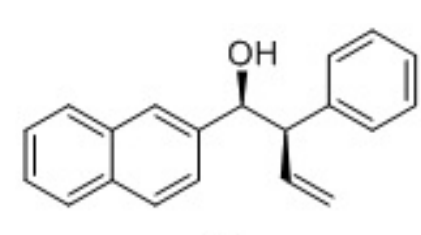

$5 \mathrm{c}$

$400 \mathrm{MHz} \mathrm{CDCl}_{3}$
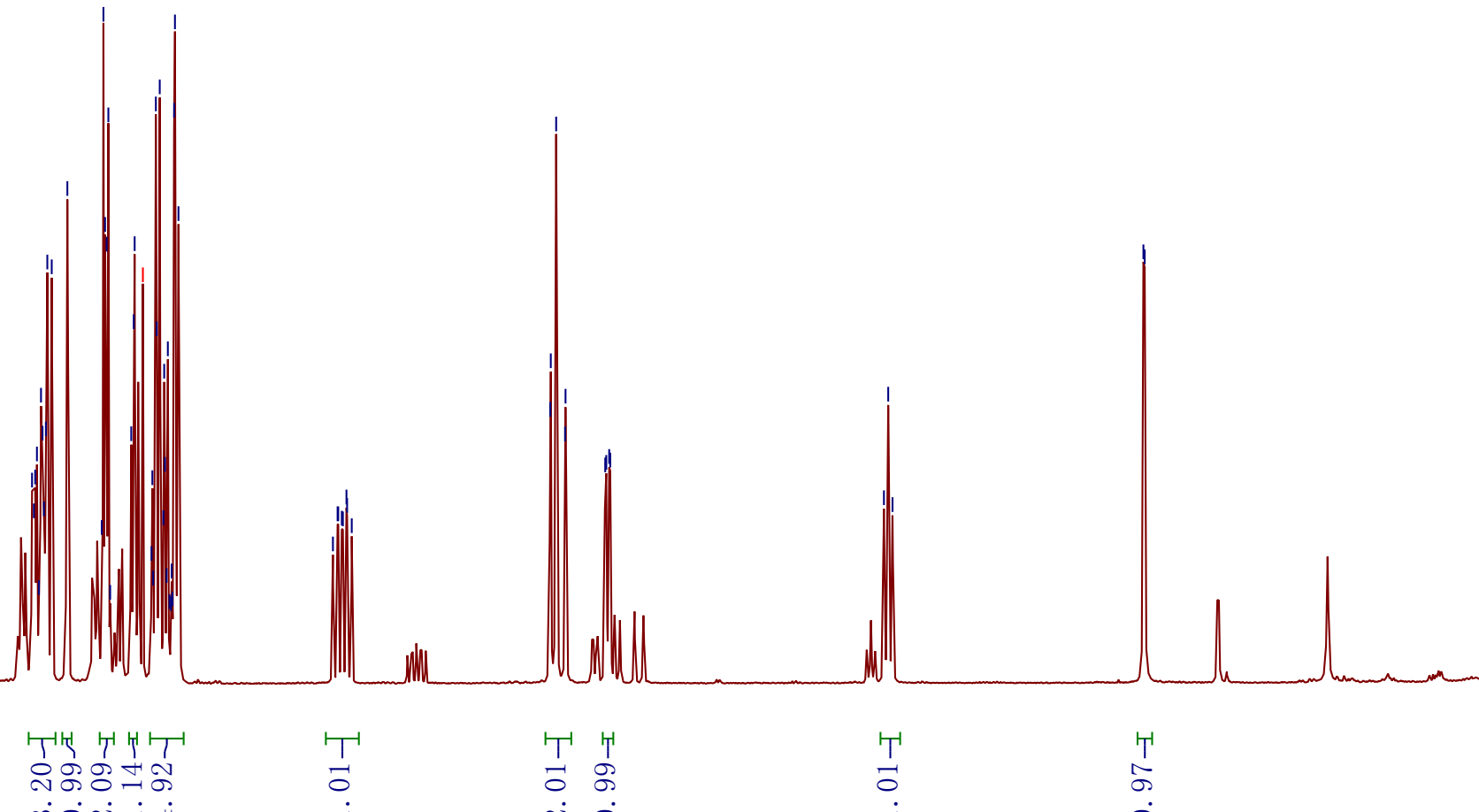

$\begin{array}{ll}T & T \\ 5 & 8\end{array}$

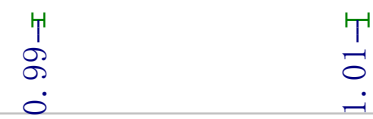

10.510 .0

5. 0 f1 (ppm)

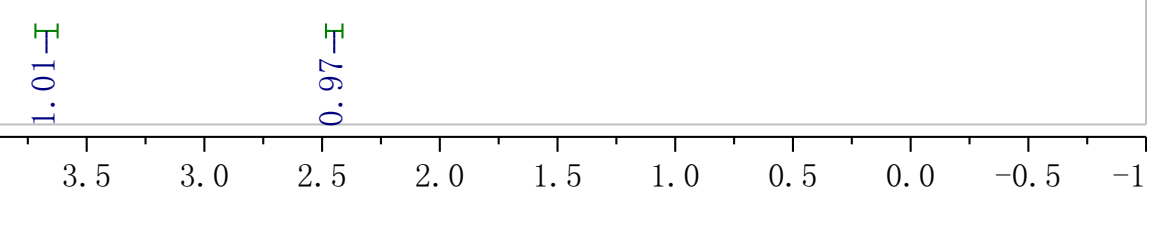




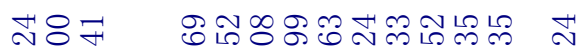

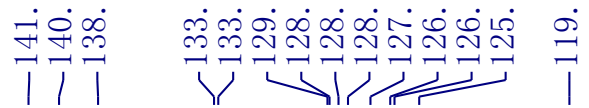

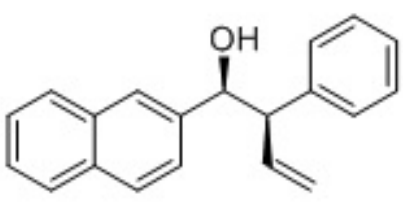

$5 \mathrm{c}$

$101 \mathrm{MHz} \mathrm{CDCl}_{3}$
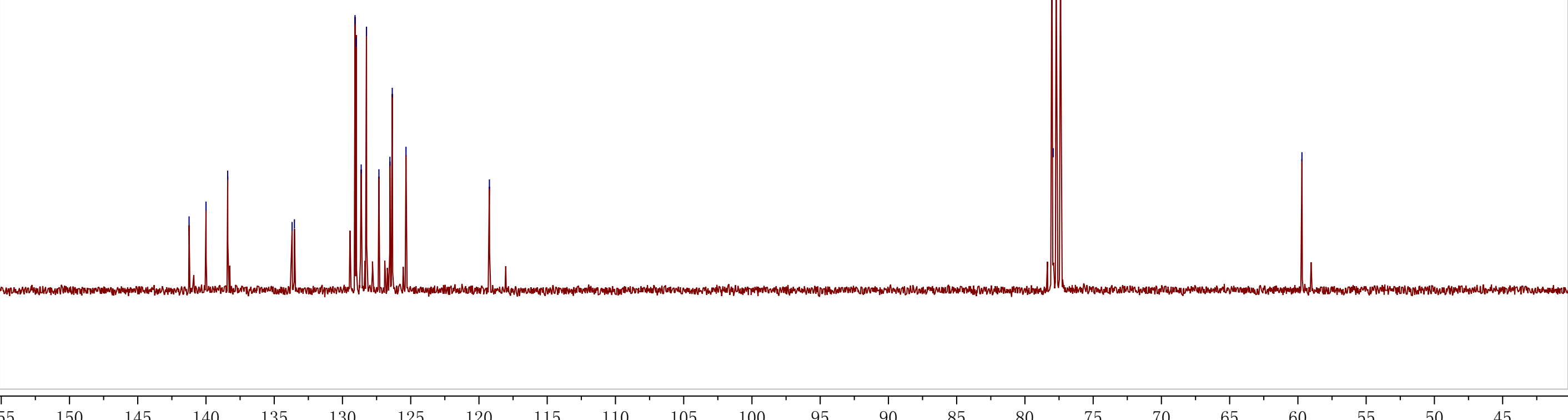

140

135

$130 \quad 125$

120

115

110

105

100



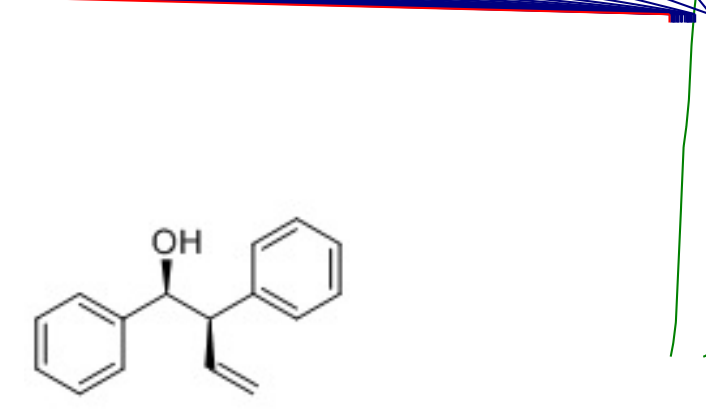

$400 \mathrm{MHz} \mathrm{CDCl}_{3}$

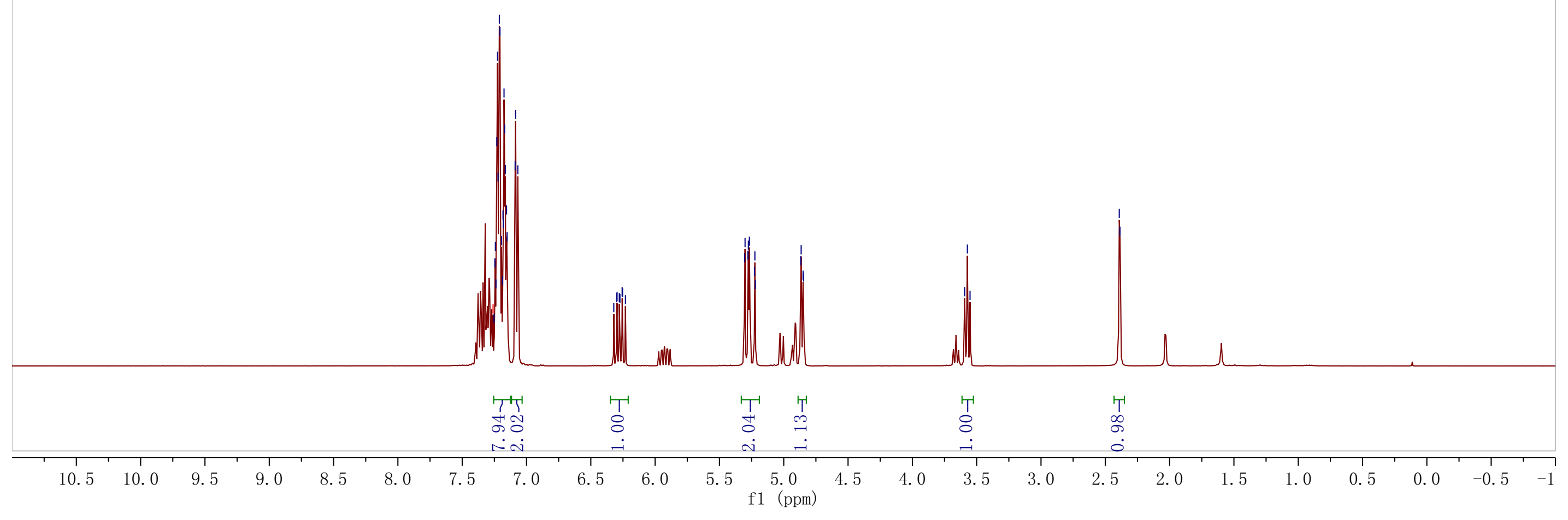




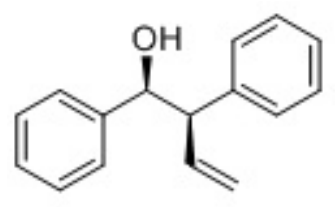

$5 d$

$101 \mathrm{MHz} \mathrm{CDCl}_{3}$

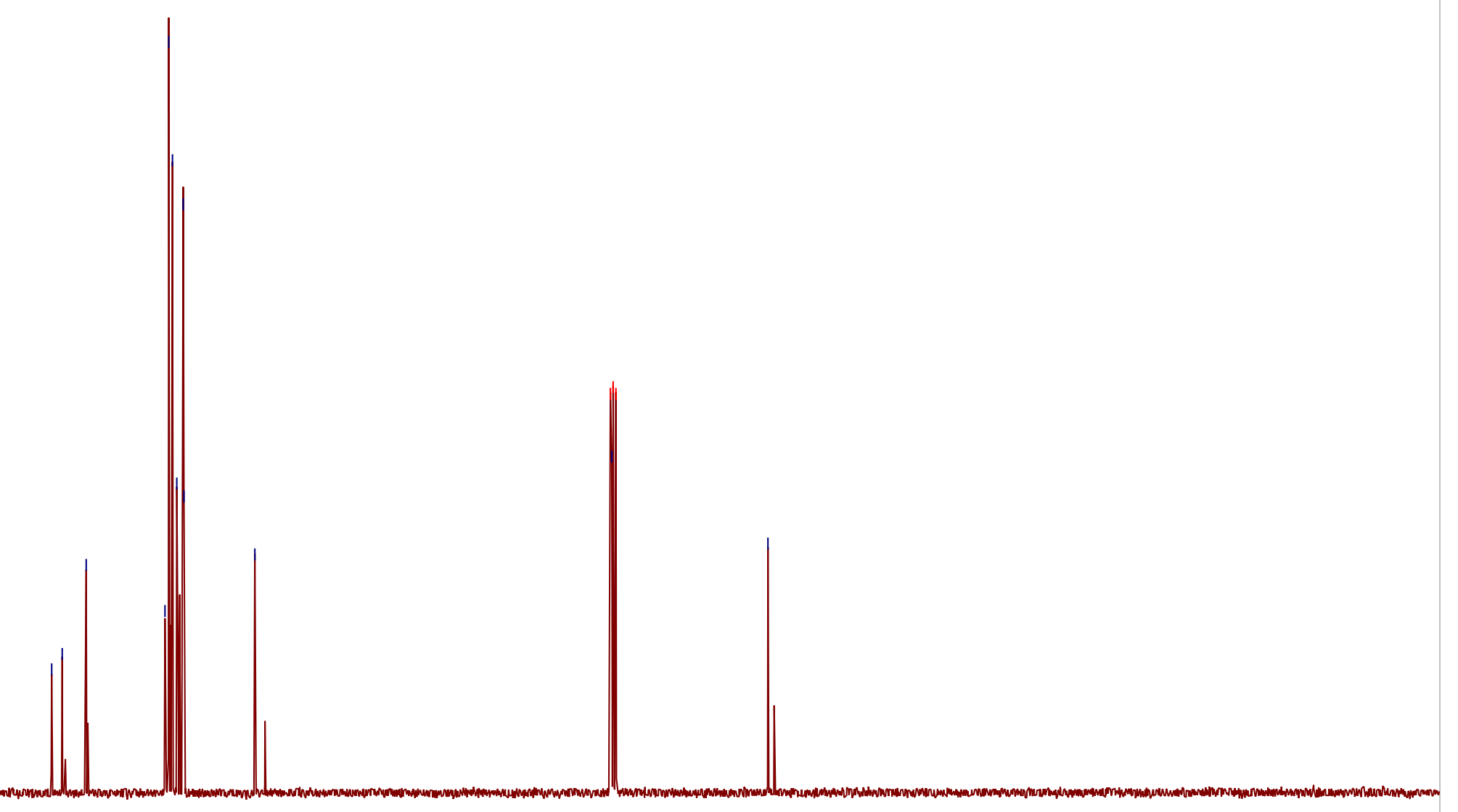



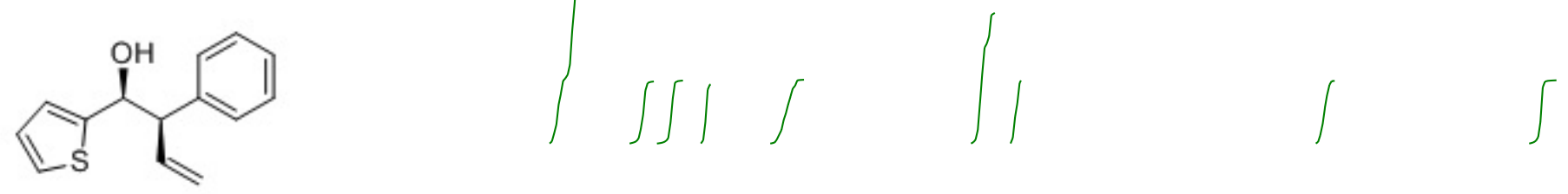

$400 \mathrm{MHz}^{\mathrm{CDCl}} 3$

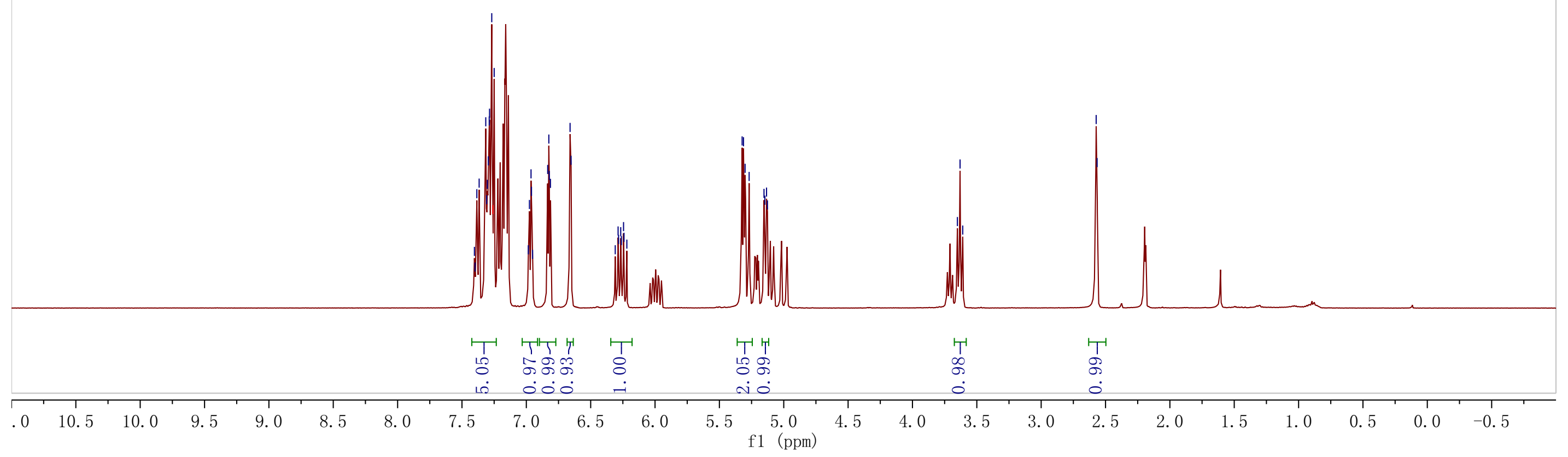




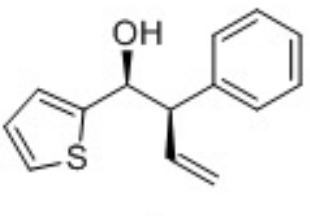

5 e

$101 \mathrm{MHz} \mathrm{CDCl}_{3}$

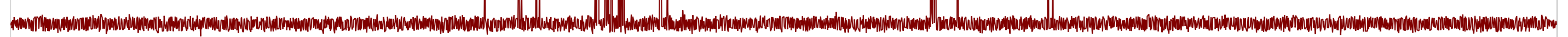

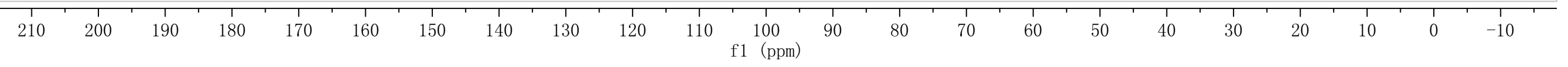



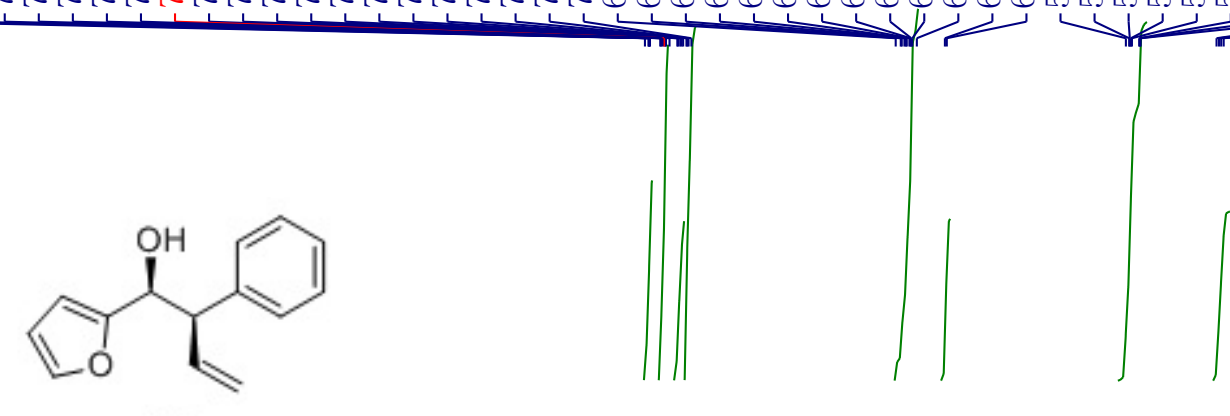

$5 f$

$400 \mathrm{MHz} \mathrm{CDCl}_{3}$

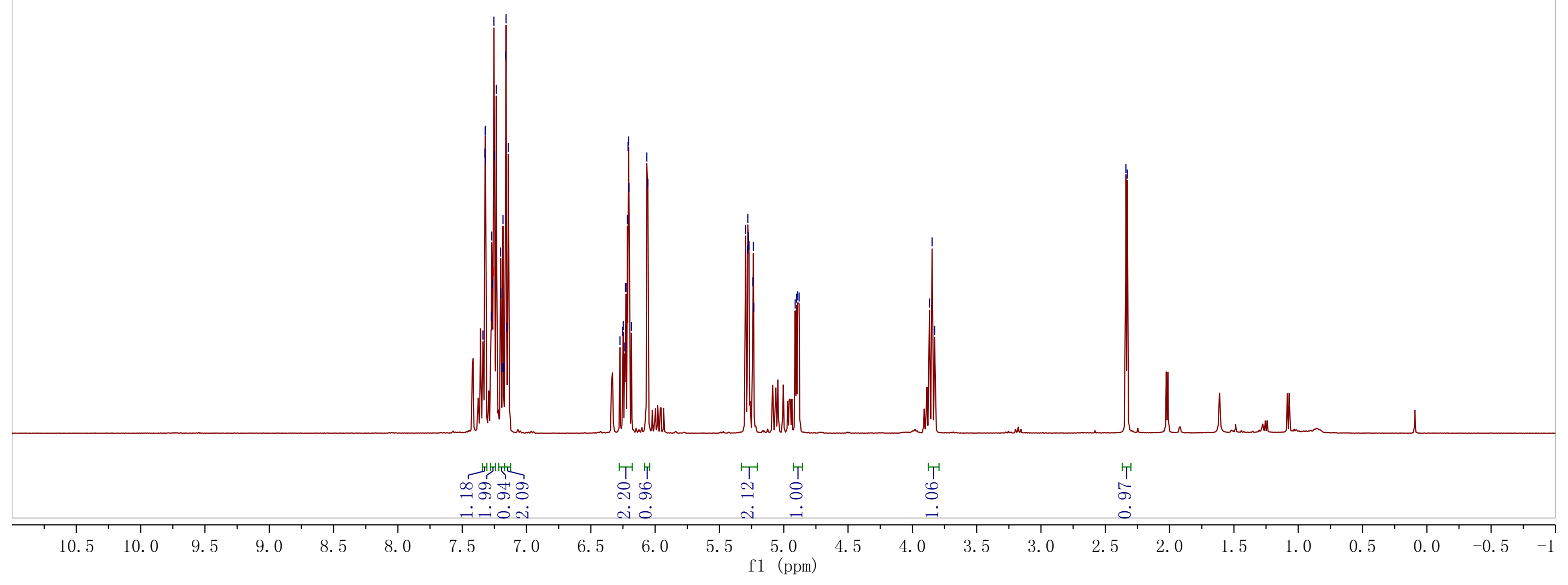




\begin{tabular}{|c|c|c|c|c|}
\hline 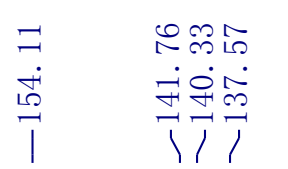 & 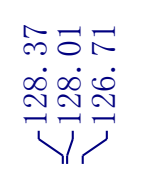 & $\begin{array}{l}\stackrel{\Im}{\sharp} \\
\infty \\
\stackrel{\infty}{=}\end{array}$ & $\begin{array}{l}8.0 \\
8.0 \\
0.0 \\
70 \\
11\end{array}$ & 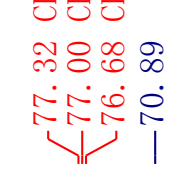 \\
\hline
\end{tabular}

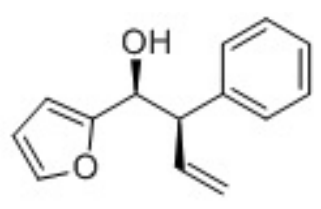

$5 \mathbf{f}$

$101 \mathrm{MHz} \mathrm{CDCl}_{3}$

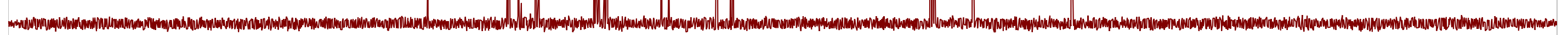

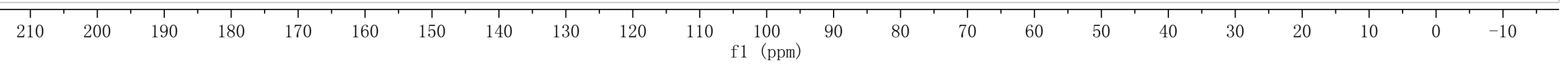




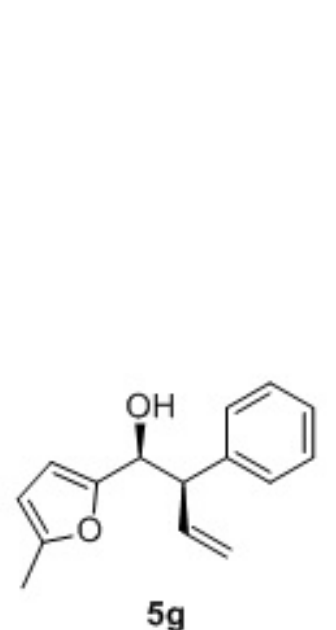

$00 \mathrm{MHz} \mathrm{CDCl}_{3}$
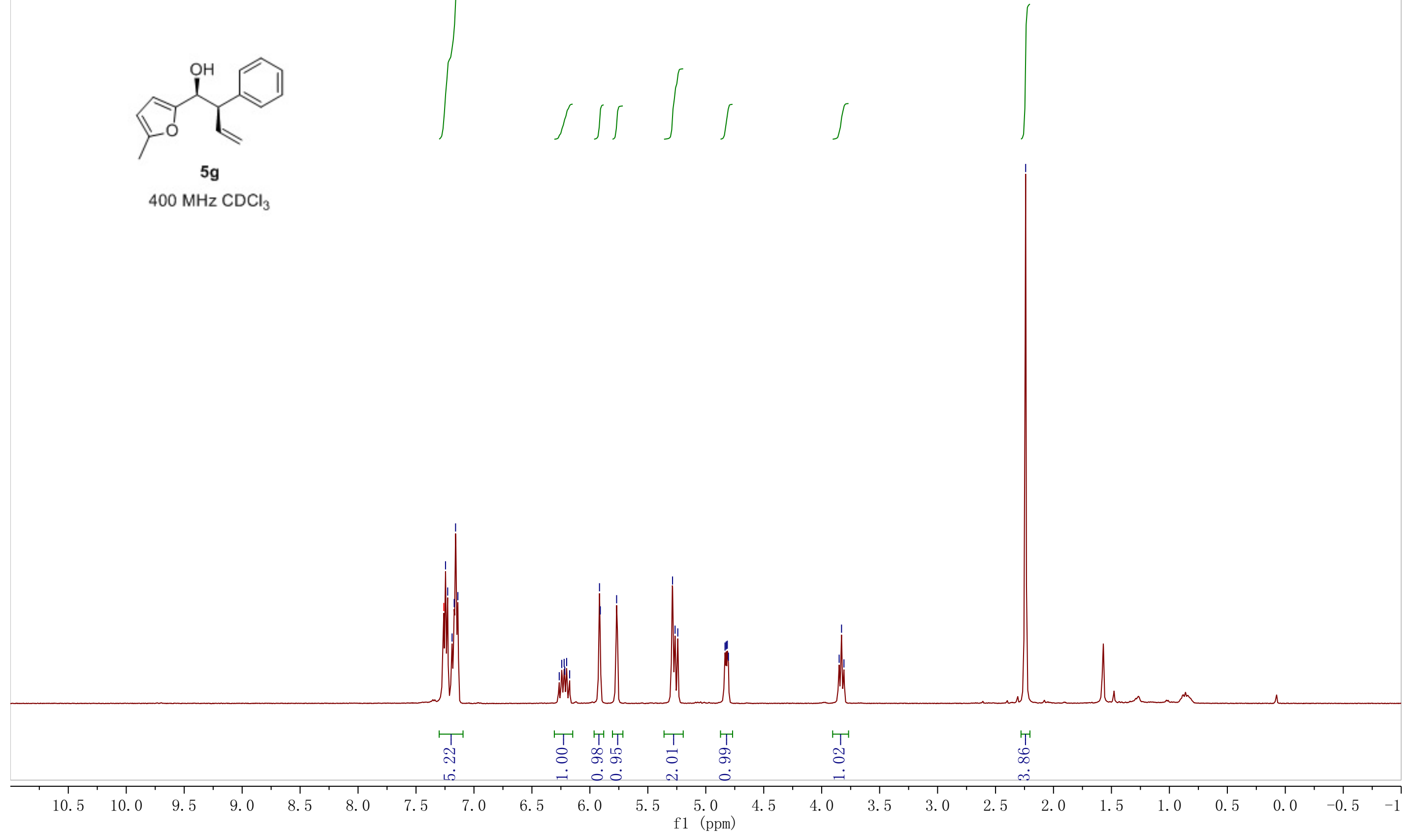


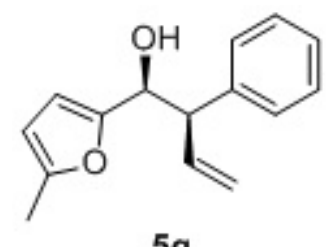

$5 g$

$101 \mathrm{MHz} \mathrm{CDCl}_{3}$

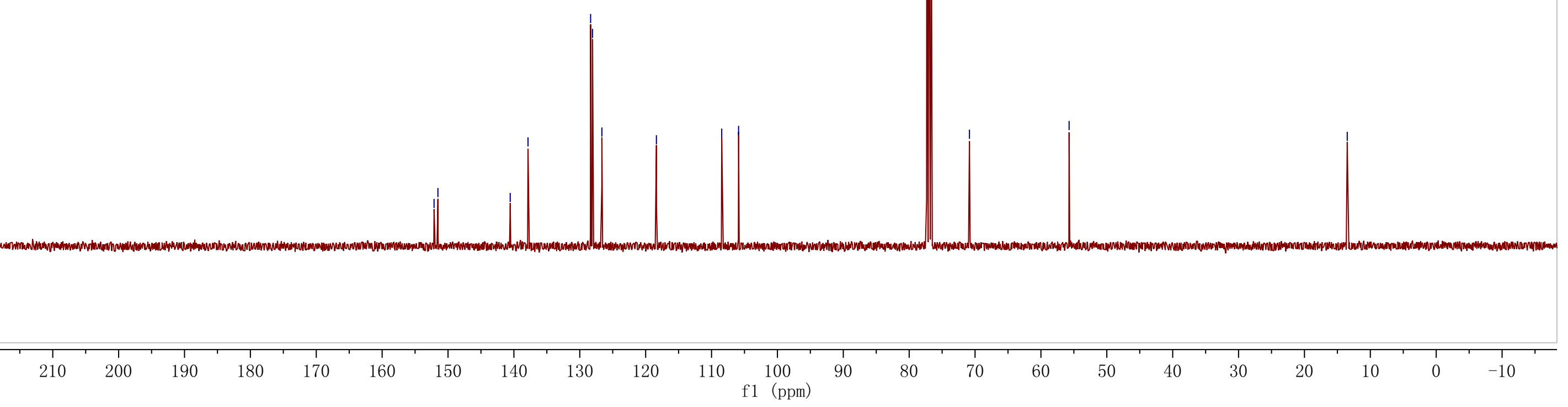




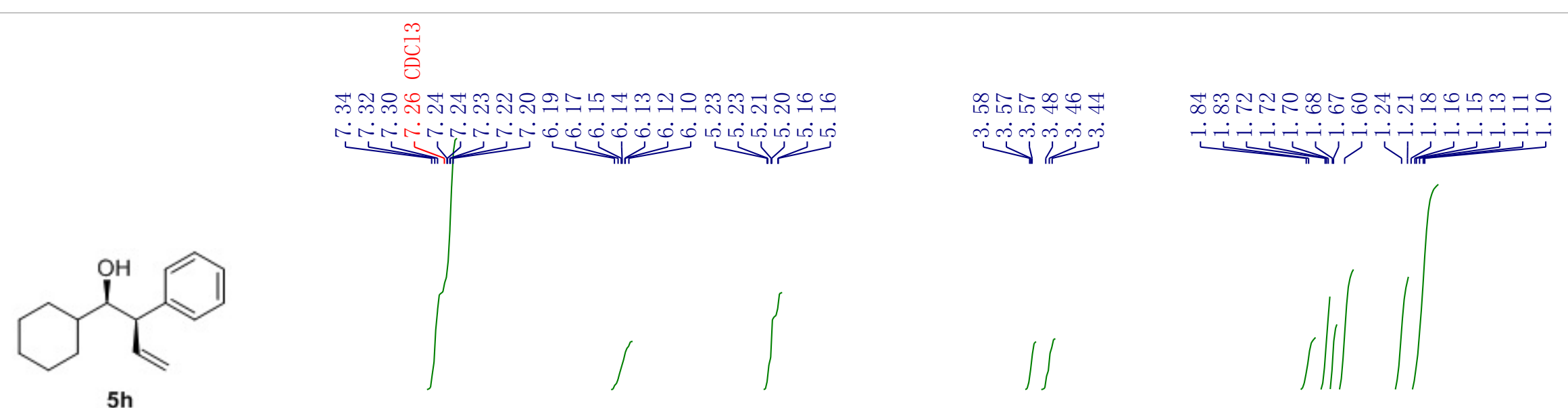

$400 \mathrm{MHz} \mathrm{CDCl}_{3}$

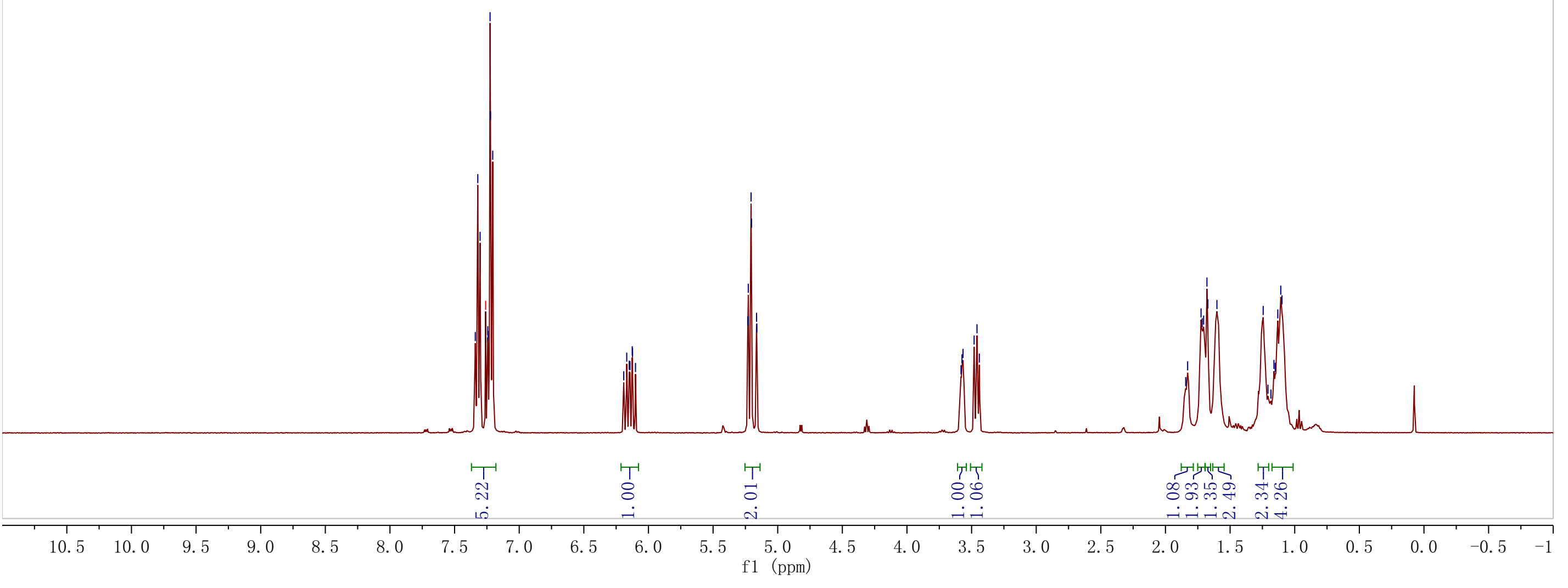




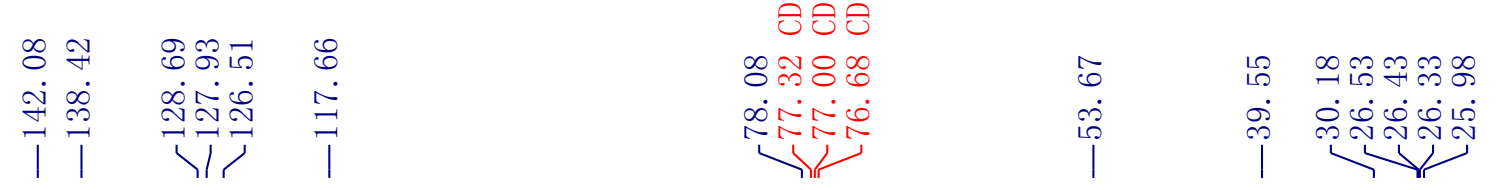

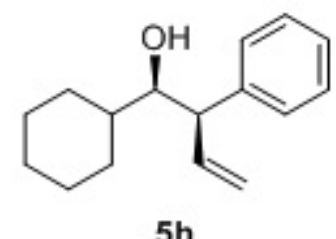

$101 \mathrm{MHz} \mathrm{CDCl}_{3}$
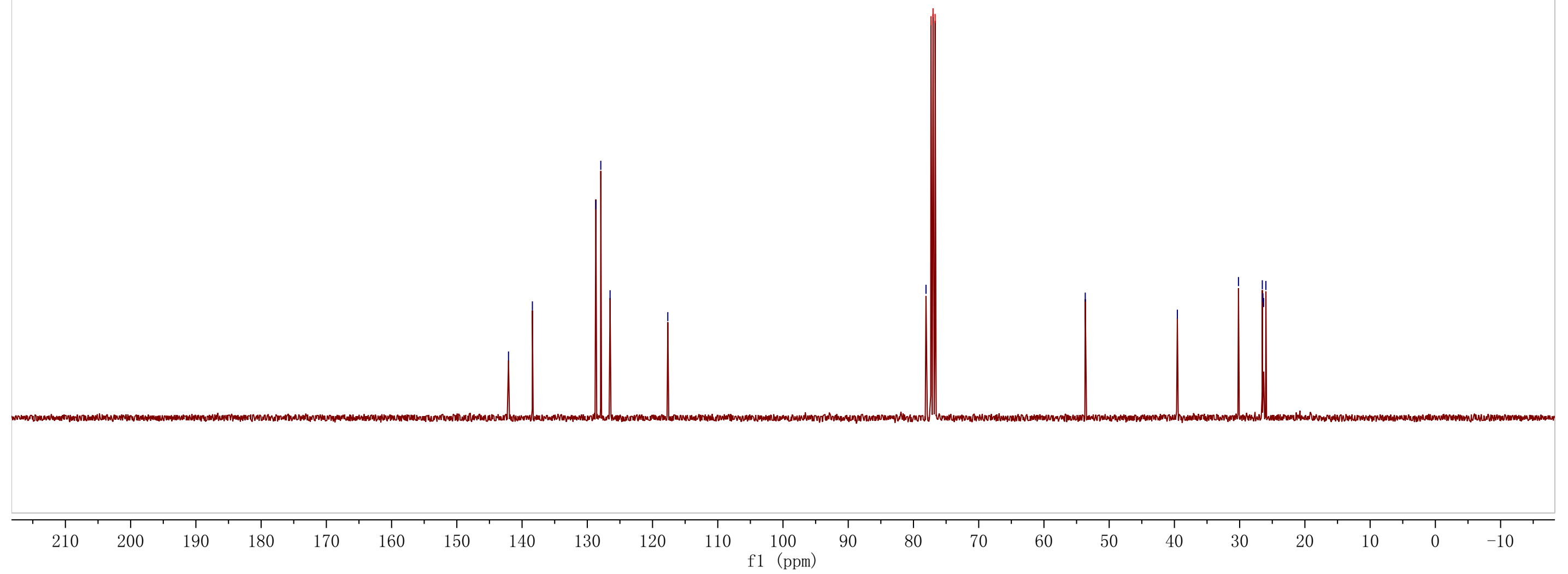


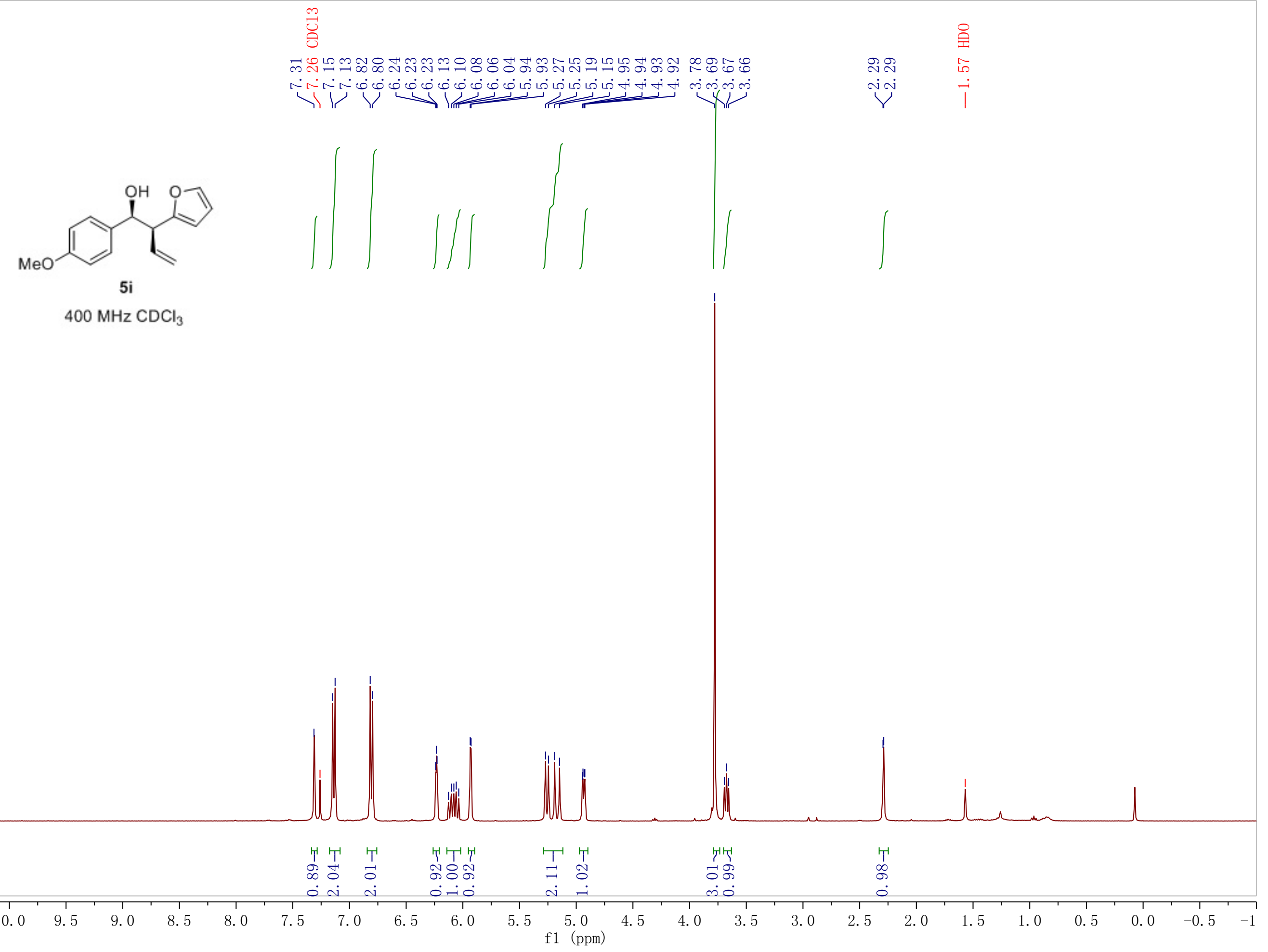




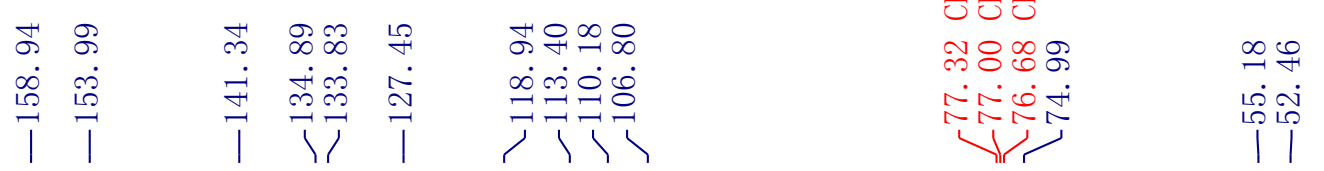

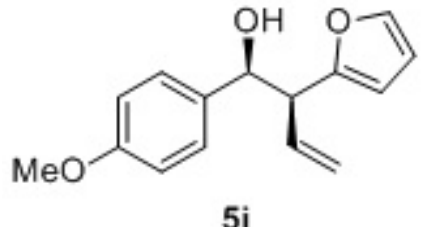

$101 \mathrm{MHz} \mathrm{CDCl}_{3}$

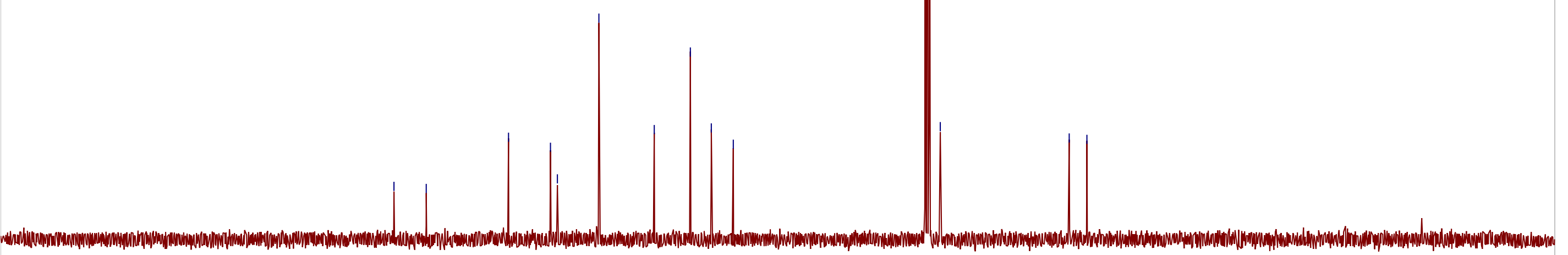

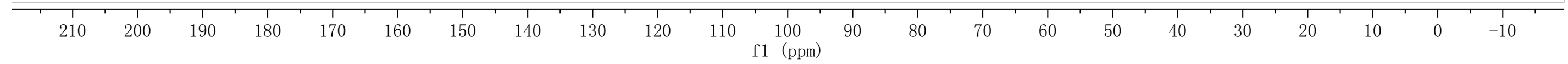




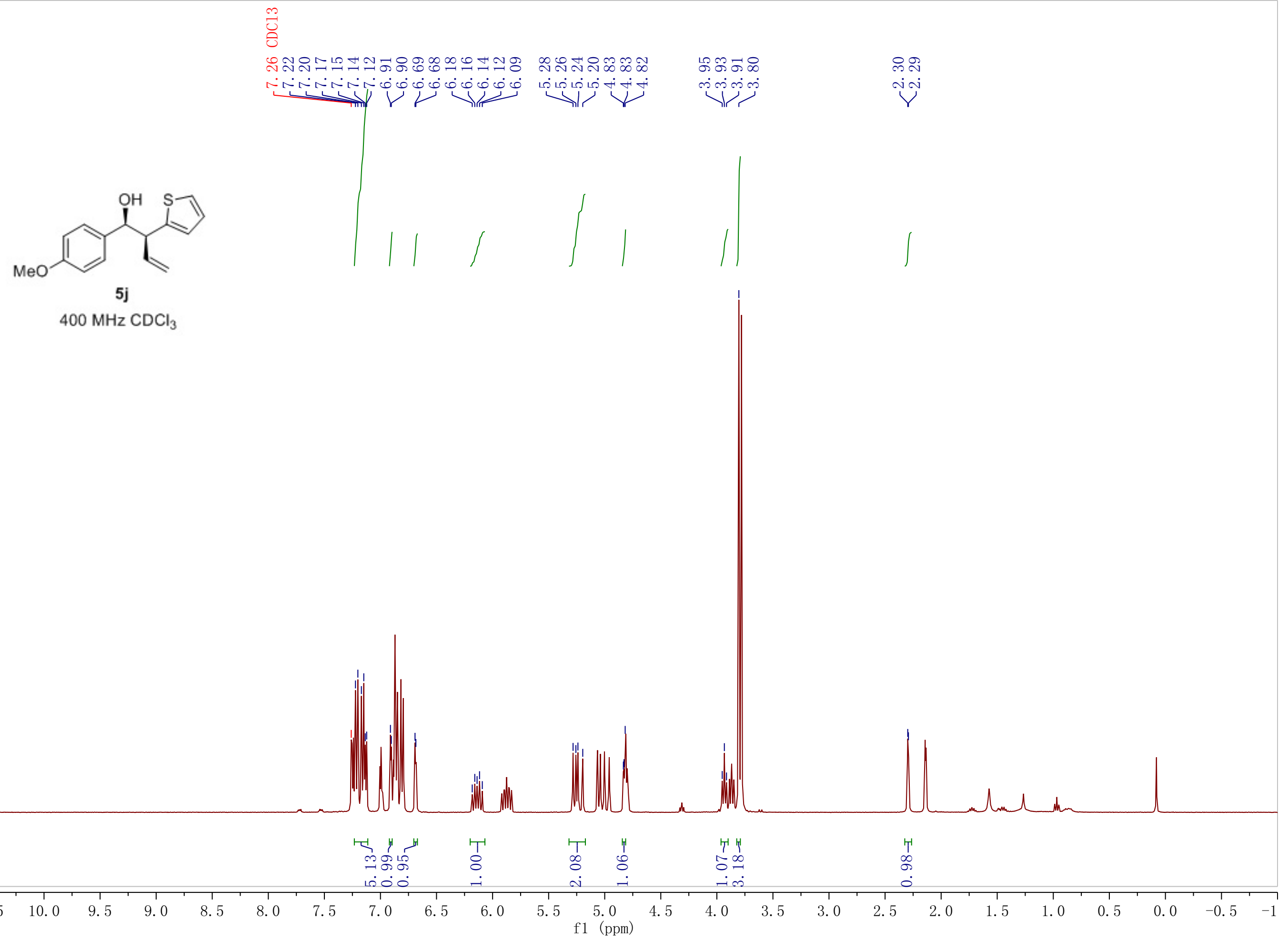




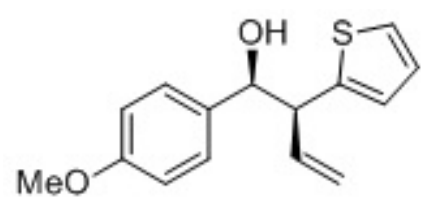

5j

$101 \mathrm{MHz} \mathrm{CDCl}_{3}$
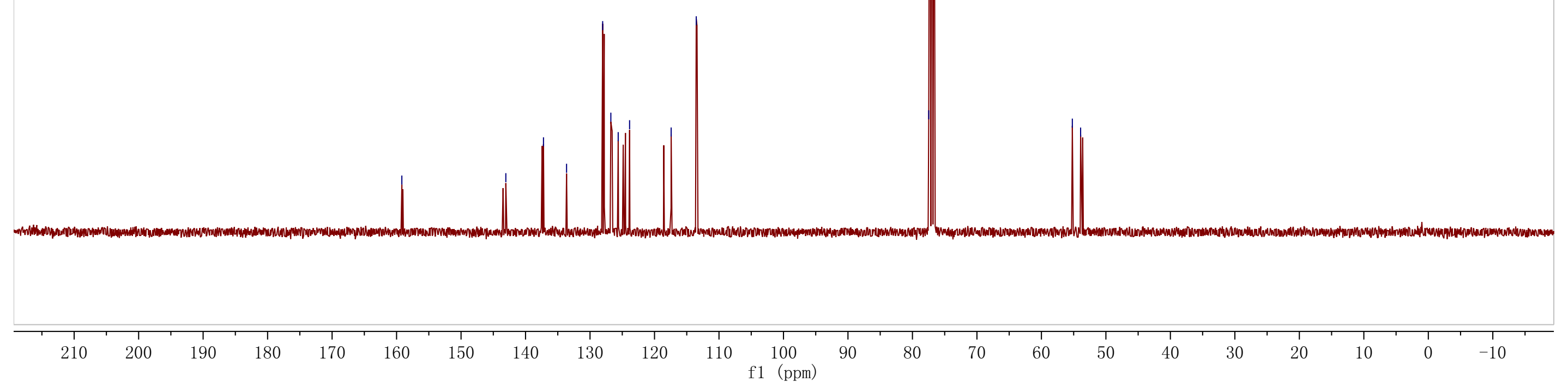


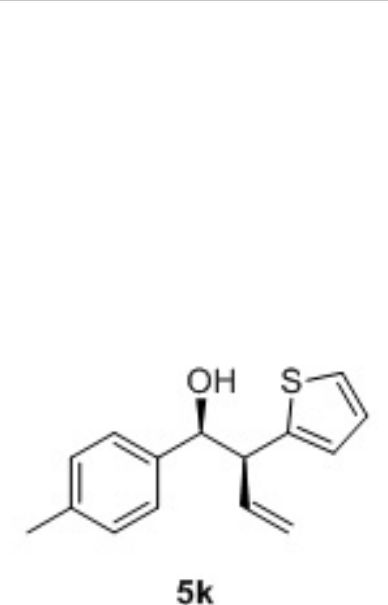

$400 \mathrm{MHz} \mathrm{CDCl}_{3}$
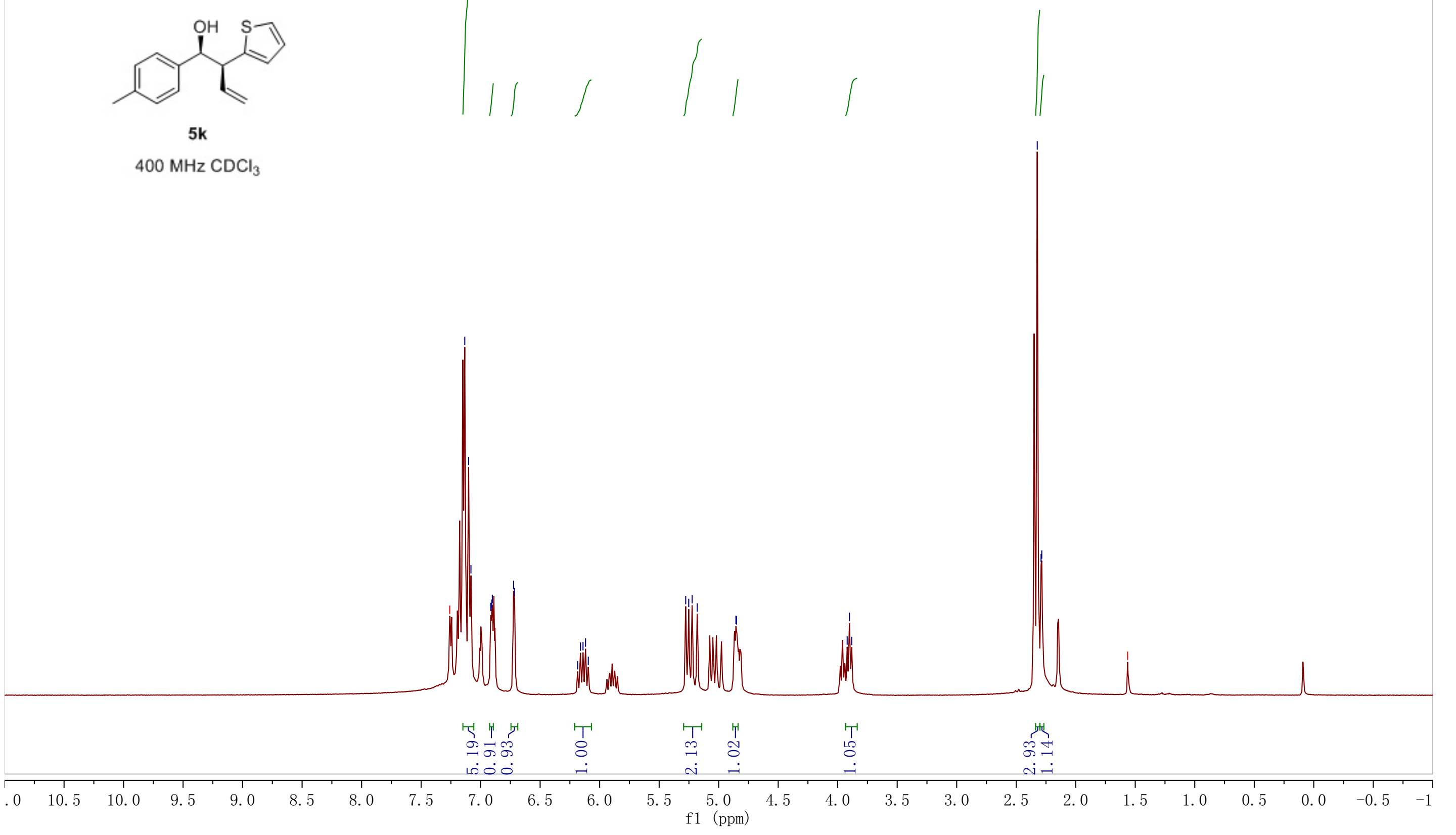


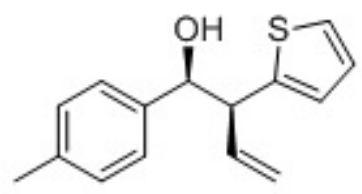

$5 k$

$101 \mathrm{MHz} \mathrm{CDCl}_{3}$

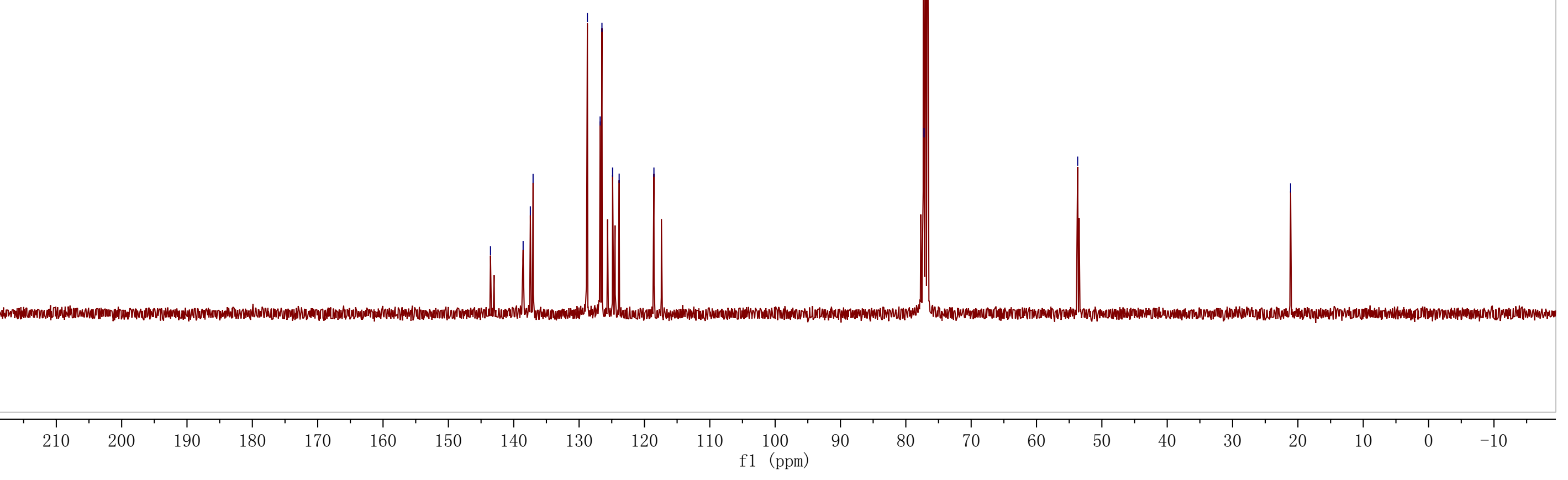




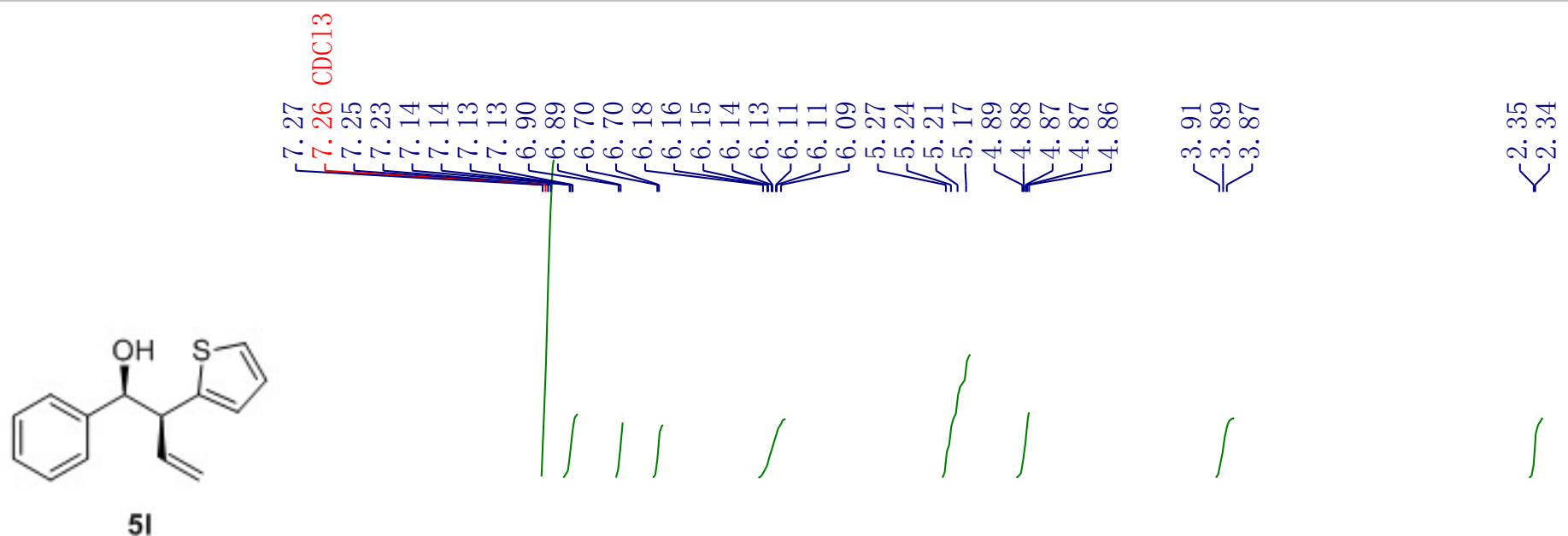

$400 \mathrm{MHz} \mathrm{CDCl}_{3}$

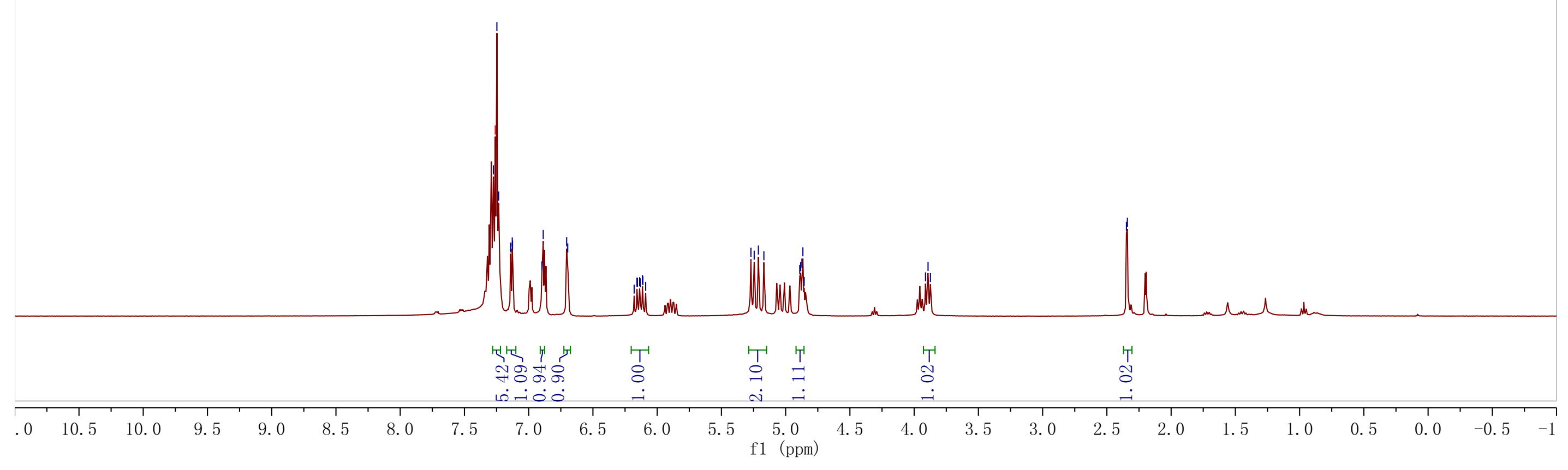




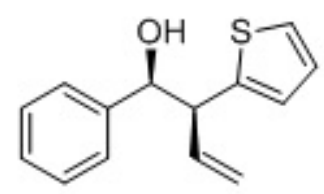

51

$101 \mathrm{MHz}^{\mathrm{CDCl}}{ }_{3}$

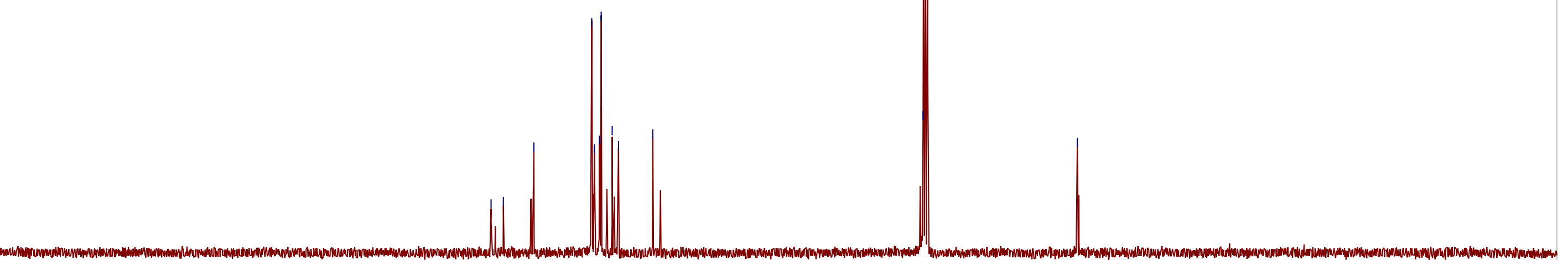

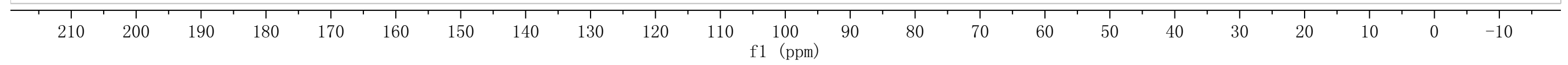


$\stackrel{\infty}{\infty} \infty \bar{\infty}$ रंग

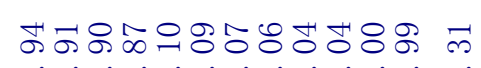

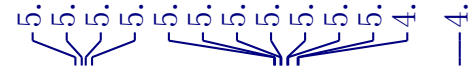

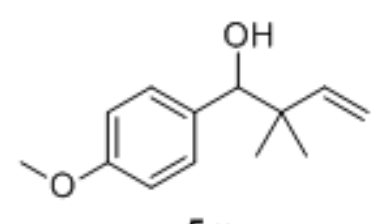

$5 \mathrm{~m}$ $400 \mathrm{MHz} \mathrm{CDCl}_{3}$

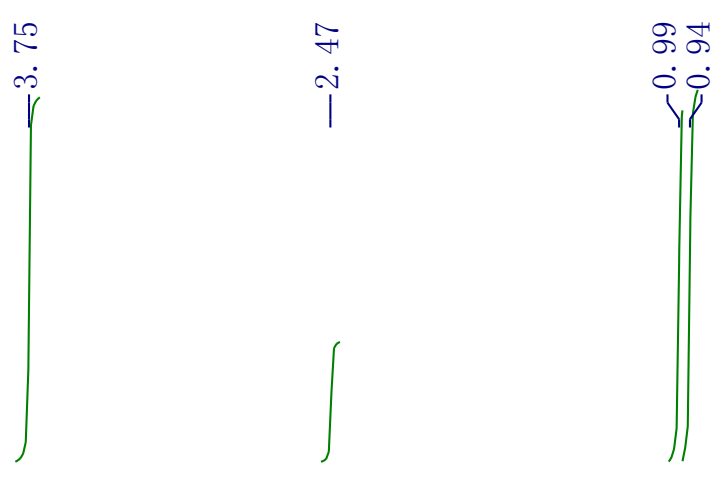

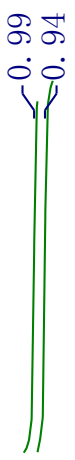

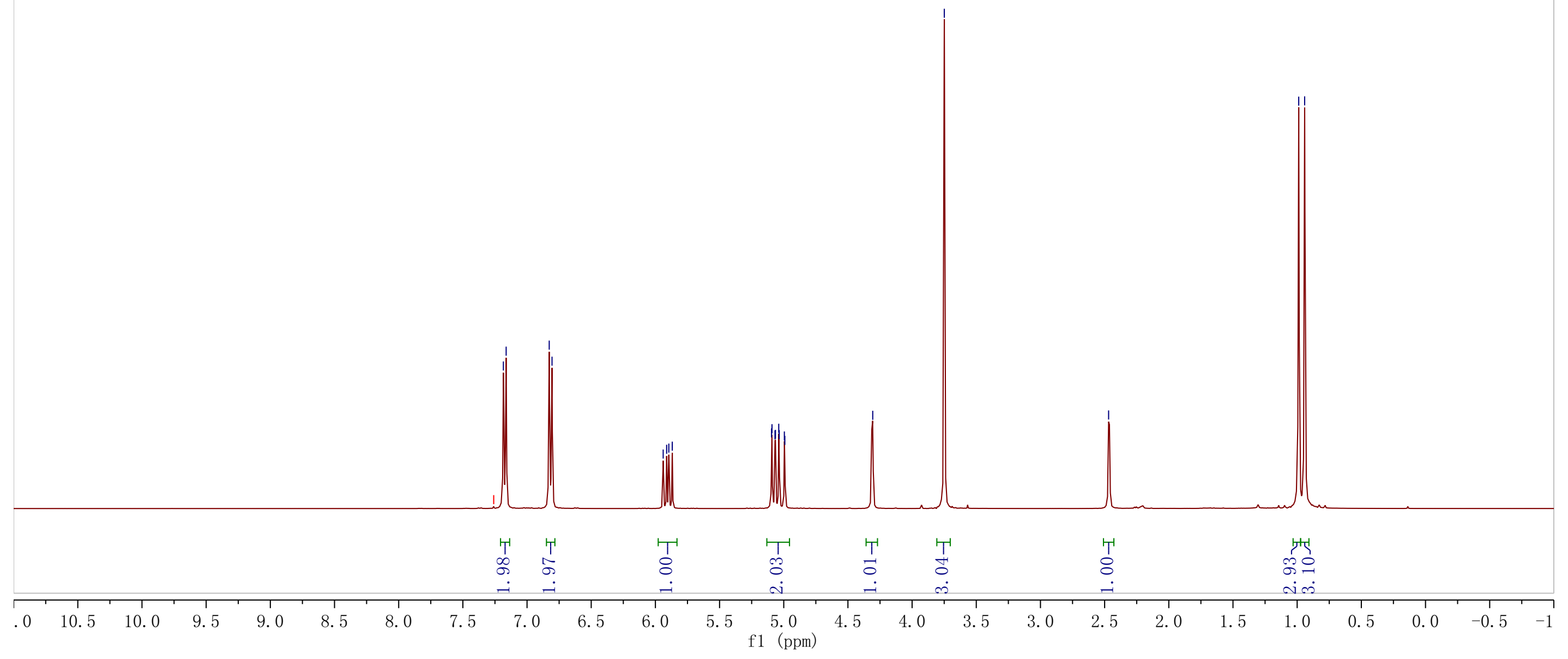




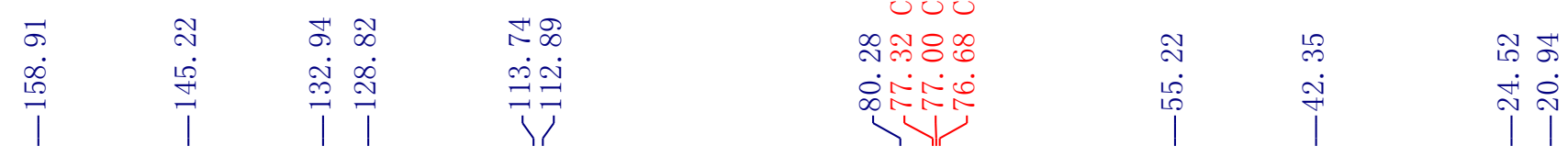

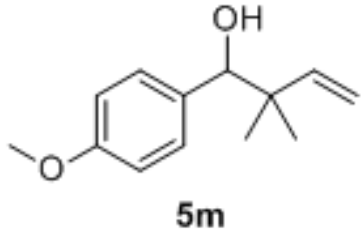

$101 \mathrm{MHz} \mathrm{CDCl}_{3}$ 